Portland State University

PDXScholar

Summer 8-8-2014

\title{
Racism, Heterosexism, Depression, and HIV Risk Behaviors of Native Men Who Have Sex With Men: Findings from the HONOR Project
}

Matthew Alan Town

Portland State University

Follow this and additional works at: https://pdxscholar.library.pdx.edu/open_access_etds

Part of the Indigenous Studies Commons, Lesbian, Gay, Bisexual, and Transgender Studies Commons, and the Sociology Commons

Let us know how access to this document benefits you.

\section{Recommended Citation}

Town, Matthew Alan, "Racism, Heterosexism, Depression, and HIV Risk Behaviors of Native Men Who Have Sex With Men: Findings from the HONOR Project" (2014). Dissertations and Theses. Paper 1947. https://doi.org/10.15760/etd.1946

This Dissertation is brought to you for free and open access. It has been accepted for inclusion in Dissertations and Theses by an authorized administrator of PDXScholar. Please contact us if we can make this document more accessible: pdxscholar@pdx.edu. 
Racism, Heterosexism, Depression, and HIV Risk Behaviors of Native Men Who Have Sex with Men: Findings from The HONOR Project

by

Matthew Alan Town

A dissertation submitted in partial fulfillment of the requirements for the degree of

Doctor of Philosophy

in

Sociology

\author{
Dissertation Committee: \\ Matthew Carlson, Chair \\ Stephanie Farquhar \\ Kelly Gonzales \\ Maura Kelly \\ Hyeyoung Woo
}

Portland State University

2014 
(C) 2014 Matthew Alan Town 


\section{ABSTRACT}

Racial minority men who have sex with men (MSM) experience greater levels of discrimination and higher rates of HIV infection. However, little is known about the associations between racial and heterosexist discrimination and HIV risk behavior. Further, little is known about the mechanisms of the association between racial and heterosexist discrimination and HIV risk behavior. There is some evidence to suggest that depression may be a mechanism that mediates the relationship between racial and heterosexist discrimination and HIV risk behavior. Thus, one purpose of this study was to investigate the extent to which discrimination based on both race and sexual orientation, alone and in combination, are associated with HIV risk behavior. A secondary purpose of this study was to examine whether the relationship between discrimination and HIV risk behavior is mediated by depressive symptoms. Lastly this study sought to examine whether the relationships between discrimination, depressive symptoms, and HIV risk behavior were mediated by social support, LGBT and Native identity, and LGBT and Native community participation.

This study analyzed data from the HONOR project, the first national study of two-spirit individuals, which included 221 American Indian and Alaska Native MSM. Logistic regression analyses were conducted to determine the extent to which experiences of racial and heterosexist discrimination were associated with HIV risk behavior. Results indicate that heterosexist discrimination was associated with HIV risk behaviors, whereas racial discrimination was not. Conversely, results indicate 
that racial discrimination was associated with depressive symptoms, whereas heterosexist discrimination was not. This study found no association between depressive symptoms and HIV risk behavior, even when accounting for alcohol and substance use. Results indicate that depressive symptoms are not a mechanism that explains the association between discrimination and HIV risk and perhaps the better mechanism to examine in future studies is substance use. Finally, LGBT community participation was shown to have protective effects against HIV risk behaviors.

These findings have the potential to guide development of mental health and HIV prevention interventions for Native MSM, with special attention to LGBT community participation and social support. Future research should examine attributes such as types, sources, and frequency of heterosexist discrimination and LGBT community participation. 


\section{ACKNOWLEDGMENTS}

There are many whose work, support, and assistance I have needed, relied upon, and appreciated. I thank my advisor Professor Matthew Carlson for his encouragement and support over the years. For their feedback, ideas, and thoughts I would like to thank my committee members: Professor Matthew Carlson, Associate Professor Stephanie Farquhar, Assistant Professor Kelly Gonzales, Assistant Professor Maura Kelly, and Associate Professor Hyeyoung Woo. I need to thank all of the other sociology faculty that I have bugged for a "quick question" and who have generously shared their time to guide and mentor me. For keeping everyone in line, I'd like to thank Bahar Jaberi and Kris Adams.

I acknowledge the help of my fellow graduate students whose struggles, discussions, and friendship have also informed this work. For their thoughtful comments I wish to thank the following colleagues and friends: Janeth Mosquera Becerra, Heather Guevara, Kimberly Ulmer Langston, Jennifer Loomis, and Caroline Smith. I also thank Kym Bradley, Michelle Holliday, Amanda Mercier, Maura Pisciotta, Jennifer Rainer, Jeff Shelton, Patrick Smith, and Daniel Standridge for their support. I will be forever thankful to my colleague and dear friend, Tina Burdsall. If it weren't for Tina, graduate school would have been even lonelier and I'm not sure I would have survived. Tina, I could not have asked for a better person to be my role model as a scientist, educator, mentor, and friend.

None of this work would have been possible without the support of many outside of Portland State University. I'd like to thank Dr. Thomas Becker, Tanya Firemoon, and the rest of the team at the Northwest Native American Research 
Center for Health for their on-going support over the years. I would also like to acknowledge Dr. William Lambert, Dr. Victoria Warren-Mears, and Joe Finkbonner for their assistance and support in returning to school. A special thanks is due to the American Sociological Association's Minority Fellowship Program and the Alpha Kappa Delta Sociology Honor Society for their generous support. I'd like to give a special yakoke to Dr. Karina Walters for not only allowing me to use data from the HONOR Project for my dissertation, but for her strong mentorship and guidance. Karina, you have been instrumental in the development of my confidence both academically and culturally. I am forever grateful.

I have had constant family support from several different families. I'd like to first acknowledge those that have come before me and those that have yet to come. I need to acknowledge and give special thanks to my parents, Carrie and Alan Town as well as my sister and brother-n-law, Sarah and Trevor Hutchens. I also need to thank my grandmothers, Millie Moffenbier and Jane Town and as well as my aunties, uncles, and cousins. I'd also like to thank my family from the Indigenous Wellness Research Institute. The support from my friends and colleagues there has been overwhelming. Specifically, I'd like to thank: Shanondora Billiot, Danica Brown, Tessa Evans-Campbell, Angela Fernandez, Jessica Lepak, Jordan Lewis, Roberto Orellana, Raven Ross, Katie Schultz, Ciwang Teyra, and Karina Walters (the ISMARTies).

Lastly, I want to acknowledge those that go unnamed, your guidance and support do not go unnoticed. 


\section{TABLE OF CONTENTS}

ABSTRACT

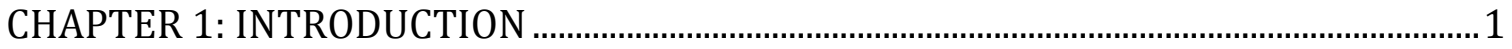

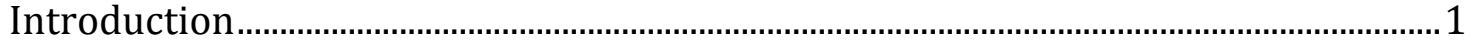

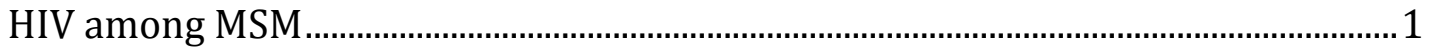

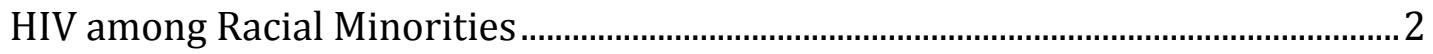

HIV among Racial Minority MSM ..............................................................................

American Indians and Alaska Natives, Native MSM, and Two-Spirits....................... 4

HIV among Natives and Native MSM.............................................................................

Public Health Interventions to Eliminate the HIV Epidemic ........................................... 7

Social Determinants of Health ......................................................................................... 8

Cultural Determinants of Health..................................................................................... 10

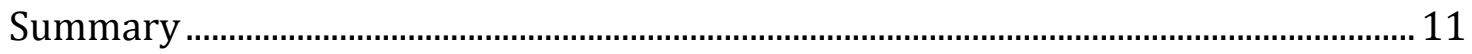

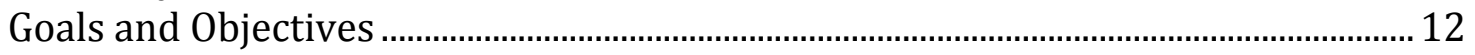

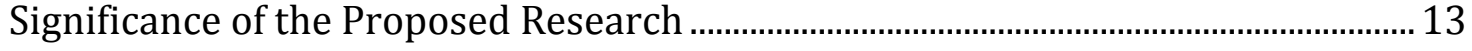

Overview of the Dissertation.............................................................................................. 13

CHAPTER 2: LITERATURE REVIEW …………………………………………………....15

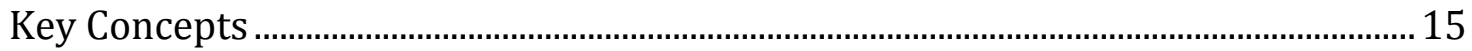

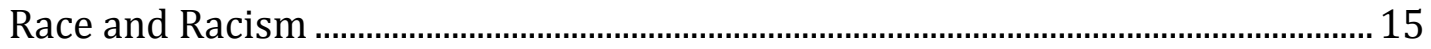

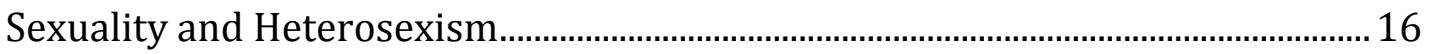

Racial/Sexual Minorities and the Intersections of Racism and Heterosexism ... 17

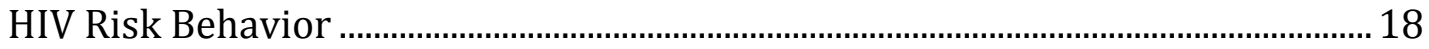

Discrimination and HIV Risk Behavior …………………...........................................2 20

The Role of HIV Status in HIV Risk Behavior..............................................................22

The Role of Partner Status in HIV Risk Behavior.......................................................2.

Discrimination and Mental Health ...................................................................................28

Depression and HIV Risk Behavior................................................................................. 33

Depression and HIV Risk among Black MSM............................................................ 34

Depression and HIV Risk among Asian and Pacific Islander MSM............................ 35

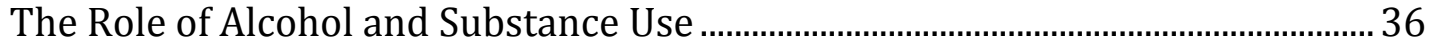

Influences of Cultural Determinants............................................................................. 40

Overview of Cultural Determinants.............................................................................. 40

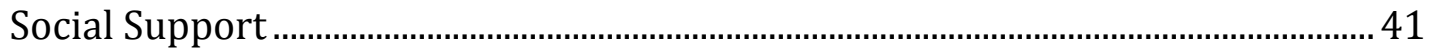

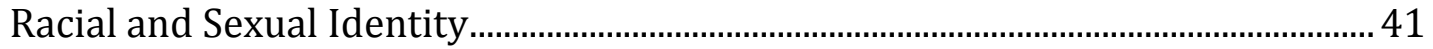

Racial and Sexual Community Participation.............................................................. 43

Other Associations and HIV Risk Behavior ...................................................................... 45

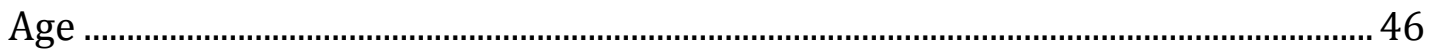

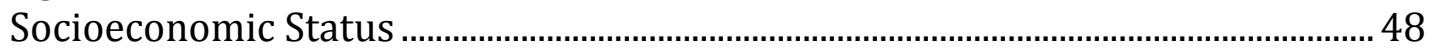


Educational Attainment......................................................................................... 49

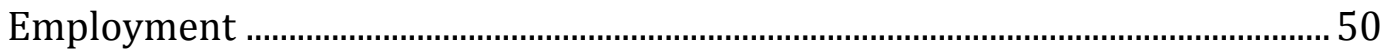

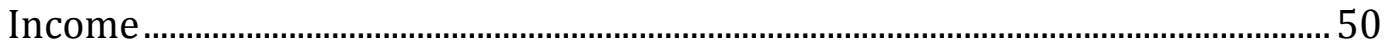

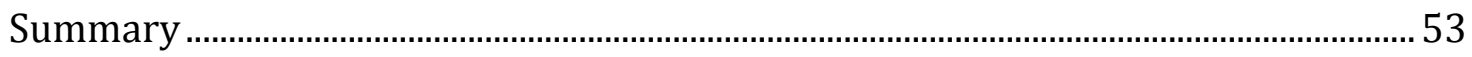

CHAPTER 3: THEORETICAL REVIEW...............................................................................56

Contextualizing Two-Spirits ...................................................................................56

Theoretical Framework: Linking Discrimination to HIV Risk Behaviors ................. 62

Linking Discrimination to Depression ........................................................................65

Linking Depression to Maladaptive Coping Behaviors .................................................69

Linking Cultural Determinants to Depression \& Maladaptive Coping Behaviors.. 70

Demographic Factors.....................................................................................................73

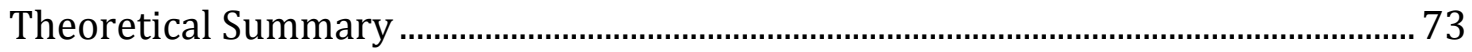

Research Questions for this Study ………………………………………………...... 74

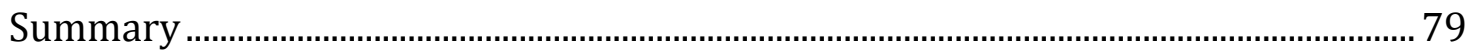

CHAPTER 4: METHODOLOGY …………………………………………………………...

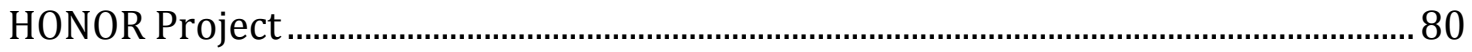

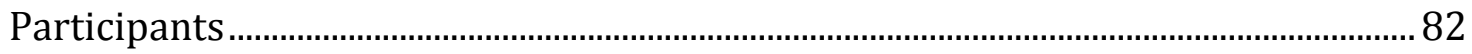

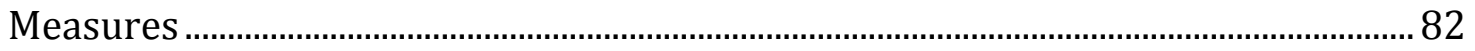

Demographic Controls............................................................................................... 82

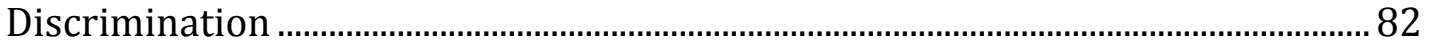

HIV Risk Behavior …………………………………………………………………….. 84

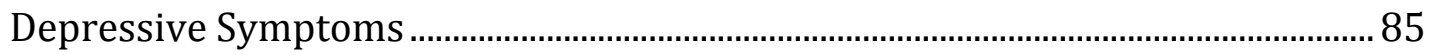

Alcohol and Substance Use........................................................................................ 85

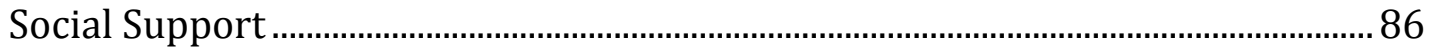

LGBT and Native Identities ....................................................................................... 87

LGBT and Native Community Participation................................................................ 88

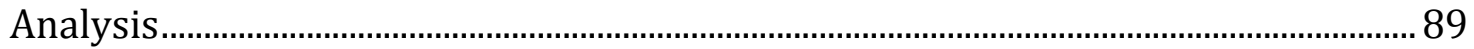

Analysis of Specific Aims and Research Questions ...................................................... 91

Specific Aims 1 \& 3 .............................................................................................. 91

Specific Aim 2 …………………………………………………………………... 92

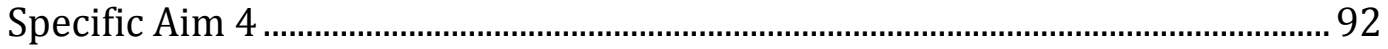

CHAPTER 5: RESULTS ..............................................................................................

Descriptive Information .......................................................................................... 94

Specific Aim 1 ………………………………………………………………..... 97

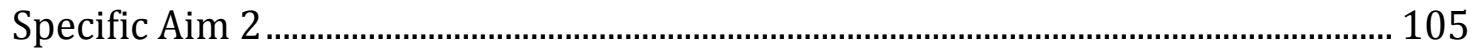

Specific Aim 3 ………………………………………………………………... 110

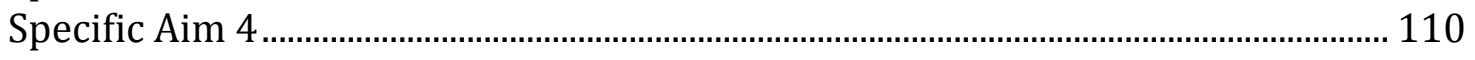

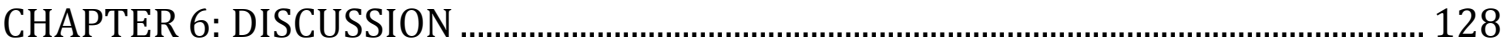

Discrimination and HIV Risk Behaviors ..................................................................... 129

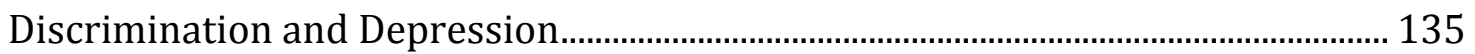

Depression and HIV Risk Behavior................................................................................ 138 


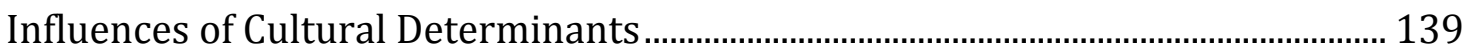

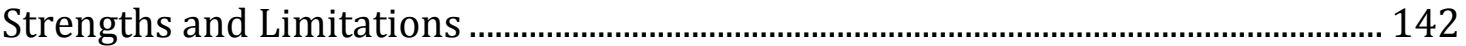

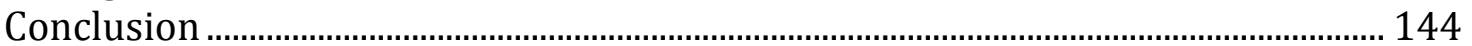

Implications and Future Directions................................................................................. 145

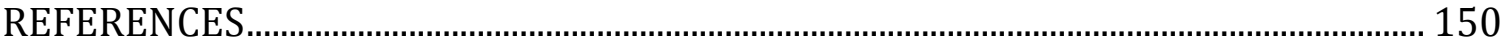

APPENDIX A: Microaggression Scales ............................................................................ 176

APPENDIX B: Center for Epidemiologic Studies - Short Depression Scale................ 179

APPENDIX C: Perceived Availability of Social Support Scale ......................................... 180

APPENDIX D: LGBT and Native Identity Scales.............................................................. 181

APPENDIX E: LGBT and Native Community Participation Measures........................... 183 


\section{LIST OF TABLES}

TABLE 1:

HIV Infection Health Disparities - Male HIV Infection by Year and Transmission

Category, 2008-2011, United States.

TABLE 2:

HIV Infection Health Disparities - HIV Infection by Year and Race, 2008-2011, United States

TABLE 3:

Distribution of Demographic and Psychosocial Characteristics of Native Mean Who Have Sex with Men, By Unprotected Anal Intercourse and Unprotected Receptive Anal Intercourse: The HONOR Project, 2005-2007

\section{TABLE 4:}

Distribution of Psychosocial and Behavioral Characteristics of Native Mean Who Have Sex with Men, By Unprotected Anal Intercourse and Unprotected Receptive Anal Intercourse: The HONOR Project, 2005-2007.

\section{TABLE 5:}

Logistic Regression Models of Native Men Who Have Sex With Men, Predicting Effects of Heterosexist and Racial Discrimination on Unprotected Anal Intercourse: The HONOR Project, 2005-2007

\section{TABLE 6:}

Logistic Regression Models of Native Men Who Have Sex With Men, Predicting Effects of Heterosexist and Racial Discrimination on Unprotected Receptive Anal Intercourse: The HONOR Project, 2005-2007

\section{TABLE 7:}

Linear Regression Models of Native Men Who Have Sex With Men, Predicting Effects of Heterosexist and Racial Discrimination on Depressive Symptoms: The HONOR Project, 2005-2007.

\section{TABLE 8:}

Logistic Regression Models of Native Men Who Have Sex With Men, Mediating Effects of Cultural Buffers on the Association Between Heterosexist and Racial Discrimination on Unprotected Anal Intercourse: The HONOR Project, 2005-2007 


\section{TABLE 9:}

Logistic Regression Models of Native Men Who Have Sex With Men, Mediating Effects of Cultural Buffers on the Association Between Heterosexist and Racial Discrimination on Unprotected Receptive Anal Intercourse: The HONOR Project, 2005-2007.

TABLE 10:

Linear Regression Models of Native Men Who Have Sex With Men, Mediating Effects of Cultural Buffers on the Association Between Heterosexist Discrimination and Racial Discrimination on Depressive Symptoms: The HONOR Project, 2005-2007. 


\section{LIST OF FIGURES}

\section{FIGURE 1:}

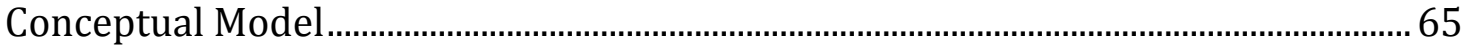


It is likely that the moment of HIV transmission into a body is a very private moment. Yet the chosen or imposed action that facilitates transmission hardly measures the enormous public forces that culminate in this irrevocable transference of viral particles. (Zierler and Krieger, 1998)

\section{CHAPTER 1: INTRODUCTION}

As the HIV epidemic sunsets its third decade, men who have sex with men (MSM) and racial minorities continue to experience a greater burden of disease and mortality from HIV. The reasons for these persistent health disparities are not well understood. The lack of understanding inhibits our ability to mitigate HIV risk behavior and improve the health and lives of MSM, racial minorities, and racial minority MSM, specifically American Indian and Alaska Native MSM.

The purpose of the following sections is to highlight HIV infection among MSM, racial minorities, racial minority MSM, American Indian and Alaska Natives, and American Indian and Alaska Native MSM.

\section{HIV among MSM}

Men who have sex with men (MSM) represent more than half of all people living with HIV; and more than $60 \%$ of all new HIV infections in the United States are reported among MSM (Ayala, Bingham, Kim, Wheeler, \& Millett, 2012; Centers for Disease Control and Prevention, 2010; Karon, Fleming, Steketee, \& De Cock, 2001; Mizuno et al., 2012). Recent HIV surveillance data regarding the mode of transmission of HIV (Table 1) (Centers for Disease Control and Prevention, 2013), indicate that male-to-male sexual contact accounts for the largest category of 
disease transmission for men. Table 1 also shows that male-to-male sexual contact consistently accounts for the most new infections. These data also indicate a growing disparity as all other modes of transmission represented in Table 1 declined from 2008 to 2011.

Table 1: HIV Infection Health Disparities - Male HIV Infection by Year and Transmission Category, 2008-2011, United States

\begin{tabular}{|l|r|r|r|r|}
\hline & \multicolumn{1}{|c|}{2008} & \multicolumn{1}{c|}{2009} & \multicolumn{1}{c|}{2010} & \multicolumn{1}{c|}{2011} \\
\hline & \multicolumn{1}{|c|}{ No. } & \multicolumn{1}{c|}{ No. } & \multicolumn{1}{c|}{ No. } & \multicolumn{1}{c|}{ No. } \\
\hline Male-to-Male Sexual Contact (MSM) & 28,077 & 27,545 & 27,725 & 30,573 \\
\hline Injection Drug Use (IDU) & 3,069 & 2,570 & 2,305 & 2,220 \\
\hline MSM \& IDU & 1,731 & 1,547 & 1,466 & 1,407 \\
\hline Heterosexual Contacta $^{\text {a }}$ & 5,200 & 4,691 & 4,391 & 4,588 \\
\hline Other Modes of Transmission $^{b}$ & 55 & 38 & 31 & 36 \\
\hline
\end{tabular}

${ }^{a}$ Heterosexual contact with a person known to have, or to be at high risk for, HIV infection

${ }^{\mathrm{b}}$ Includes hemophilia, blood transfusion, perinatal exposure, and risk factor not reported or not identified

\section{HIV among Racial Minorities}

HIV is disproportionately distributed among racial minorities, specifically Blacks and Latinos (Karon et al., 2001; Mizuno et al., 2012). Although racial minorities comprise only one quarter of the U.S. population, they account for $58 \%$ of the HIV cases (Vernon, 2007). The most recent HIV surveillance data for all new infections, shown in Table 2 (Centers for Disease Control and Prevention, 2013), indicate that the highest incidence rate is among Blacks, followed by Latinos, populations of multiple races, and Native Hawaiian/Pacific Islanders. The lowest incidence rate is among Asians. Whites have had a consistently low incidence rate, ranging from 6.6 to 7.2 infections per 100,000 people from 2008 to 2011. American Indians and Alaska Native (Native) have also had a stable incidence rate over the same time period, ranging from 8.7 to 9.8 per 100,000 people. 
Table 2: HIV Infection Health Disparities - HIV Infection by Year and Race, 20082011, United States

\begin{tabular}{|c|c|c|c|c|c|c|c|c|}
\hline & \multicolumn{2}{|c|}{2008} & \multicolumn{2}{|c|}{2009} & \multicolumn{2}{|c|}{2010} & \multicolumn{2}{|c|}{2011} \\
\hline & No. & Rate $^{a}$ & No. & Rate $^{a}$ & No. & Rate $^{a}$ & No. & Rate $^{a}$ \\
\hline AIAN & 225 & 9.6 & 205 & 8.7 & 222 & 9.8 & 212 & 9.3 \\
\hline Asian & 816 & 6.1 & 753 & 5.5 & 780 & 5.3 & 982 & 6.5 \\
\hline Black & 24,419 & 65.4 & 22,618 & 60.0 & 22,030 & 58.0 & 23,168 & 60.4 \\
\hline Latino $^{b}$ & 9,691 & 20.6 & 9,495 & 19.6 & 9,225 & 18.2 & 10,159 & 19.5 \\
\hline $\mathrm{NH} / \mathrm{PI}$ & 79 & 17.9 & 80 & 17.9 & 62 & 12.4 & 78 & 15.3 \\
\hline White & 14,277 & 7.2 & 13,371 & 6.7 & 13,069 & 6.6 & 13,846 & 7.0 \\
\hline Multiple & 994 & 22.5 & 886 & 19.4 & 879 & 15.6 & 827 & 14.2 \\
\hline
\end{tabular}

a Rates are per 100,000 population

$\mathrm{b}_{\text {Hispanics/Latinos can be of any race }}$

\section{HIV among Racial Minority MSM}

Due to data limitations, the estimates of HIV prevalence and incidence among racial minority MSM are not well known. The data limitations stem from an underreporting of male-to-male sexual contact among men overall. One way to account for these data limitations is to use STD rates as a proxy. Racial minority MSM consistently have higher rates of STDs than their majority counterparts (Aral, Adimora, \& Fenton, 2008; Hall, Byers, Ling, \& Espinoza, 2007) suggesting an increased risk for HIV infection. As such, racial minority MSM are at increased risk of acquiring HIV (Hall et al., 2007; Halpern et al., 2004). Another way to account for data limitations is to make use of the mandatory reporting data that is collected when individuals initially test positive for HIV. For example, Black and Latino MSM have five to three times greater rates of HIV diagnosis compared to estimates reported for white MSM (Hall et al., 2007; Mojola \& Everett, 2012). 


\section{American Indian and Alaska Natives, Native MSM, and Two-Spirits}

Before discussing HIV among American Indians and Alaska Natives, it is important to clarify this population given they are the focus of this dissertation. An official or "agreed upon" definition is quite challenging due to the diversity that exists across nations and within communities. The term American Indian and Alaska Native refers to those "who have experienced the imperialism and colonization of the modern historical period... They remain culturally distinct, some with their native languages and beliefs still alive. They are minorities in territories and states over which they once held sovereignty" (Smith, 2005). The literature uses several terms that have been used traditionally, scientifically, and those preferred by American Indian and Alaska Native groups including: Native, Indigenous, Native American, American Indian, Alaska Native, American Indian and Alaska Native, and AIAN to generally describe this population. The term "Native" will be used in the remaining sections of this dissertation.

It is also important to clarify the term "Two-Spirit". Two-Spirit is a contemporary term, adopted in 1990 at the third annual spiritual gathering of lesbian, gay, bisexual and transgender natives, to signify the embodiment of both feminine and masculine spirits (Anguksuar [LaFortune R], 1997). This pan-Indian term is used contemporarily to signify diverse gender and sexual identities among American Indian, Alaska Native, and Canadian First Nations people. Indigenous activists have adopted the term as a way for gay, lesbian, bisexual, or transgendered Natives to name themselves outside the colonizing terms previously imposed upon them (e.g., "berdache"), to reconnect with tribal traditions related to sexuality and 
gender identity, and to transcend the Eurocentric binary categorizations of heterosexual versus homosexual and male versus female (Jacobs, Thomas, \& Lang, 1997). It is important to note that Native people can identify as two-spirit in addition to or instead of lesbian, gay, and bisexual as well as transgender or cisgender. The term "two-spirit" will be used in place of "American Indian and Alaska Native gender and sexual minorities" as a means of contextualizing and historicizing this population in both time and place. Additionally, the term "Native MSM" refers to American Indian and Alaska Native men who have sex with men. This distinction is to differentiate identity (two-spirit) from behavior (Native MSM).

\section{HIV among Natives and Native MSM}

There is growing attention to understand HIV among Native populations (Bertolli et al., 2004; Bertolli, Lee, \& Sullivan, 2007; Bouey \& Duran, 2000; Burks, Robbins, \& Durtschi, 2011; Cassels, Pearson, Walters, Simoni, \& Morris, 2011; Conway et al., 1992; Diamond, Davidson, Sorvillo, \& Buskin, 2001; Duran et al., 2000; R. L. Hall, Wilder, Bodenroeder, \& Hess, 1990; Kaufman et al., 2007; Lapidus, Bertolli, McGowan, \& Sullivan, 2006; McNaghten, Neal, Li, \& Fleming, 2005; Pearson, Walters, Simoni, Beltran, \& Nelson, 2013; Simoni, Sehgal, \& Walters, 2004; Simoni, Walters, Balsam, \& Meyers, 2006; Thurman, Vernon, \& Plested, 2007; Vernon, 2007; Walters, Simoni, \& Harris, 2000; Wiechelt, Gryczynski, \& Johnson, 2009). Of these studies only five examine HIV among Native MSM (Burks et al., 2011; Cassels et al., 2011; Pearson et al., 2013; Simoni et al., 2006; Walters et al., 2000). Of the few studies available, two highlight the modes of HIV exposure among HIV-positive 
Natives. Historically and consistently, male-to-male sexual contact is the most common way HIV-positive Natives have been exposed to HIV (Bertolli et al., 2004; McNaghten et al., 2005). Bertolli and colleagues (2004) reported that MSM accounted for $55 \%$ of the cumulative total of Natives with AIDS. The following year, McNaghten and colleagues (2005) reported similar findings, as the predominant mode of exposure to HIV among Native men with AIDS was having sex with other men (54.5\%), followed by injection drug use (IDU) (22.4\%), MSM/IDU (13.5\%), and heterosexual contact (8.0\%). Similarly, the predominant mode of exposure for men of other races/ethnicities with AIDS (i.e. excluding Natives) was MSM (53.7\%), IDU (26.6\%), heterosexual contact (11.6\%), and MSM/IDU (6.8\%).

Several dimensions of the HIV epidemic for Natives are especially concerning. Natives experience a significantly shorter time from initial diagnosis of HIV infection to AIDS-defining illness than any other racial group in the United States (Centers for Disease Control and Prevention, 2003b). In 2001, 48\% of Natives diagnosed with HIV were subsequently diagnosed with AIDS within 12 months, compared with $40 \%$ for the general population (Centers for Disease Control and Prevention, 2003b). Natives also experience one of the lowest survival rates after an AIDS diagnosis is made (Centers for Disease Control and Prevention, 2003a). Available data do not provide information on whether this rapid progression (and low survival) is due to a late diagnosis of HIV, or an accelerated viral pathogenesis. Among MSM, 5-year increases in AIDS incidence rates were higher for Natives (53\%) than for Blacks (45\%) or Latinos (23\%) (Sullivan, Chu, Fleming, \& Ward, 1997). Recent studies report prevalence rates of HIV infection among Native MSM 
ranging from18\%-34\% (Cassels et al., 2011; Pearson et al., 2013).

\section{PUBLIC HEALTH INTERVENTIONS TO ELIMINATION THE HIV EPIDEMIC}

Studies investigating higher rates of HIV prevalence and incidence among racial minority MSM have generally cited high-risk behavior, including unprotected sex and injection drug use as modes of transmission. Historically, most public health interventions have focused on reducing the risk of acquiring HIV by targeting individual behaviors, promoting behavior change and lifestyle modification, thereby minimizing disease occurrence (Aggleton, 2004; Parker, 2001; Phillips \& Pirkle, 2011). However, despite a great deal of effort from HIV prevention, intervention, and education programs, the annual number of new infections of HIV remains consistent and disproportionate based on racial and sexual group membership. In a review of HIV prevention interventions, Elford and Hart (2003) found that while there is evidence demonstrating that community-level interventions are effective, the effectiveness of individual-level interventions is unclear. This evidence suggests that the individual-level approach of addressing behavior change and lifestyle modification is not enough. The broader social-context that influences HIV risk, such as the social and cultural determinants of health, need to be explored further (Burks et al., 2011; Farmer, 1999, 2005; Zierler \& Krieger, 1998). Using this expanded approach may illuminate the importance of the social-context and lived experiences of racial minority MSM, specifically Native MSM, to promote HIV risk reduction. 


\section{SOCIAL DETERMINANTS OF HEALTH}

The disparities in HIV infection may be due to an incompatibility of prevention efforts with cultural norms, knowledge, and behavior patterns of targeted populations (Burks et al., 2011). One limitation of the individually focused, risk-behavior approaches is that they fail to capture the social determinants of health by placing too much emphasis on 'high risk' or 'core' groups (e.g.: MSM, IDU, etc.). The social determinants of health refer to "both specific features of and pathways by which societal conditions affect health and that potentially can be altered by informed action" (Krieger, 2001:697). A social determinants of health perspective takes into account that HIV infection is affected by patterns of sexual and drug-related behavior in a population, but this perspective also states that HIV is not produced solely by individuals' choices and behaviors. Rather, a social determinants of health perspective views HIV infection as an outcome of a combination of factors, which are in part influenced by individual behavior, but also socially and culturally produced (Health Canada, 1998; Marmot \& Wilkinson, 1999). That is to say, HIV infection is due to a number of mechanisms that enable and protect against practices and behaviors that result in transmission (Heffernan, 2002; Lane et al., 2004; Zierler \& Krieger, 1998).

Many researchers and public health practitioners have recognized the importance of addressing social determinants of health in dealing with the HIV epidemic (Dean \& Fenton, 2010; Sharpe, Harrison, \& Dean, 2010), and research addressing various types of discrimination must be part of the portfolio of such endeavors. Discrimination defined as "the process by which a member, or members, 
of a socially defined group is, or are, treated differently (especially unfairly) because of his/her/their membership of that group" (Jary \& Jary, 1995:169) is a component of the social determinants of health. Parker and Aggleton argue that discrimination needs to be (re)conceptualized as "social processes that can only be understood in relation to broader notions of power and domination" (Parker \& Aggleton, 2003:16).

Discrimination has played a central role in shaping responses to the HIV/AIDS epidemic, yet the effects of discrimination on the HIV-related health disparities have not been well examined (Poundstone, Strathdee, \& Celentano, 2004). Recent scholarship regarding racial minority MSM has demonstrated that members of these groups often experience discrimination due their race and sexuality (Battle, Cohen, Warren, Fergerson, \& Audam, 2002; Díaz, Ayala, Bein, Henne, \& Marin, 2001; Han, 2007; Ramírez, 2003; Teunis, 2007). Studies suggest that such experiences may be risk factors for problematic physical, mental, and behavioral health outcomes (Krieger, 2000; Martin, Tuch, \& Roman, 2003; Mays \& Cochran, 2001; Noh \& Kaspar, 2003; Williams, Yu, Jackson, \& Anderson, 1997). Studies focused specifically on racial minority MSM suggest that experiences of discrimination based on both sexuality and race are associated with higher levels HIV sexual risk behavior (Díaz et al., 2001; Diaz \& Ayala, 2001; Hirokazu Yoshikawa, Wilson, Chae, \& Cheng, 2004). Though the scholarship supports the association between discrimination and HIV risk behavior, researchers also address the mechanisms that help explain this association, specifically depressive symptoms. In particular, several studies demonstrate that discrimination, based on race and sexuality, is associated with depressive symptoms among racial minority MSM (Díaz 
et al., 2001; Williams, Neighbors, \& Jackson, 2003; Hirokazu Yoshikawa et al., 2004). In addition, the association between depressive symptoms and HIV risk behavior illustrates that those with diminished ability to cope with stressors partake in maladaptive coping strategies such as unprotected anal intercourse (UAI) as well as alcohol and substance use (Aneshensel, 1992; Pearlin, 1989; Schwartz \& Meyer, 2010).

\section{CULTURAL DETERMINANTS OF HEALTH}

Though still in it's infancy, researchers and public health practitioners are beginning to recognize the importance of a cultural determinants of health perspective. The cultural determinants of health perspective stems largely from studies conducted with First Nations Canadians that examines the importance of cultural identity and cultural participation on health status (Wilson \& Rosenberg, 2002). This literature attempts to operationalize culture into 'cultural buffers' (Walters, Simoni, \& Evans-Campbell, 2002; Wilson \& Rosenberg, 2002). Recent scholarship on the cultural practices of racial minority MSM demonstrates their involvement in cultural activities often within their respective racial and ethnic communities and well as within the larger LGBT community (Ratti, Bakeman, \& Peterson, 2000; Seibt et al., 1995). This literature also highlights how racial minority MSM often are at odds with community practices and expectations between their racial and sexual groups. However, studies do suggest that racial and sexual identity and racial and sexual community participation may serve as protective factors against UAI (Chng \& Geliga-Vargas, 2000; Walters et al., 2002). Social and cultural 
determinants of health perspectives have been used to examine factors that influence HIV risk behavior for Asian, Black, and Latino MSM; however, there is a dearth of research on these phenomena among Native MSM.

The contributions of social and cultural determinants of health perspectives with respect to understanding HIV/AIDS have grown in recent years. These contributions are due in part to the trend that Koopman (1996) describes as the "transition from a science that identifies risk factors for disease to one that analyzes the systems that generate patterns of disease in populations" (p. 630). This transition has led to developments that have reconceptualized HIV risk and in doing so have led to investigations into HIV that better match the realities of HIV transmission (Poundstone et al., 2004). The challenge, however, is for prevention and intervention research to not treat behavior as decontextualized and dehistoricized phenomena. Those conducting HIV prevention, intervention, and research need to be cognizant of social and cultural determinants of HIV risk behavior, such as unprotected anal intercourse, that account for large numbers of people acquiring and living with HIV (Phillips \& Pirkle, 2011).

\section{SUMMARY}

To continue to be effective in response to the HIV epidemic, public health efforts must conceptualize HIV risk beyond individual behavior, as this approach can lead toward framing of HIV infection as a failure of individuals in high risk populations (i.e. racial minorities, MSM, and or racial minority MSM)(Caldwell, 2000) resulting in 'blaming the victim' (Farmer, 1999, 2005). Instead, public health 
efforts need to lead toward frameworks that include both social and cultural determinants of health. By understanding the social and cultural determinants that influence behavior related to HIV risk, researchers and public health practitioners will be better equipped to design and implement interventions that meet the needs of racial minority MSM. Furthermore, social and cultural perspectives contextualize the lives of racial minority MSM, including Native MSM, in a place where information can be better understood and behavior change can take place.

\section{GOALS and OBJECTIVES}

The overarching goal of this study was to investigate the extent to which discrimination based on both race and sexual orientation, alone and in combination, are associated with HIV risk behavior. A secondary purpose of this study was to examine whether the relationship between discrimination and HIV risk behavior is mediated by depressive symptoms. Lastly this study sought to examine whether the relationships between discrimination, depressive symptoms, and HIV risk behavior were mediated by social support, LGBT and Native identity, and LGBT and Native community participation. In order to achieve these goals, data on Native MSM were drawn from the HONOR Project. This study was organized using the following four specific aims:

1. Examine the extent to which discrimination is associated with HIV risk behaviors.

2. Examine the extent to which discrimination is associated with depressive symptoms. 
3. Examine the extent to which depressive symptoms are associated with HIV risk behavior.

4. Examine the extent to which social support, racial and sexual identity, and racial and sexual community participation mediate the relationships between discrimination, depressive symptoms, and HIV risk behaviors.

\section{SIGNIFICANCE OF THE PROPOSED RESEARCH}

The proposed research will contribute to the health literature in two ways. First the proposed study will explore the specific experiences of Native MSM that are currently lacking from the larger debate on the impact of discrimination and HIV risk behavior among racial minority MSM. Second, the proposed study seeks to use a framework based on the social and cultural determinants of health as they relate specifically to Native MSM. Using this framework provides a better understanding of the lived experiences of Native MSM and how these lived experiences influence HIV risk behavior.

\section{OVERVIEW OF THE DISSERTATION}

This dissertation begins with a review of the literature in Chapter Two, first by addressing and clarifying key concepts and then reviewing the literature pertaining to racial minority MSM as they relate to the specific aims outlined above. Chapter Three builds a theoretical framework for the association between discrimination and HIV risk behavior incorporating minority stress and 'Indigenist' stress-coping theories. Chapter Three ends with the research questions and 
hypotheses. Chapter Four describes the proposed research methodology, measures, and analysis plan. Chapter Five summarizes of the results. The dissertation concludes with a discussion of the findings, strengths and limitations, and implications for interventions and future research. 


\section{CHAPTER 2: LITERATURE REVIEW}

The purpose of this chapter is to review the literature corresponding with the specific aims from the previous chapter. This chapter is organized into six sections. The first section is an overview of key concepts. The second section is a review of the associations of discrimination and HIV risk behavior. The third section reviews the literature of the association between discrimination, depression and HIV risk behavior. The fourth section reviews the association between depression and HIV risk behaviors. The fifth section reviews the effects of cultural buffers on the associations between discrimination, depression, and HIV risk behavior and the final section reviews demographic factors and their relationship with HIV risk behaviors. Due to the dearth of research these associations among Native MSM, I review empirical research pertaining to these associations in other racial minority MSM populations.

\section{KEY CONCEPTS}

\section{Race and Racism}

Race is a complex concept, best viewed for social science purposes not as a biological construct reflecting innate differences, but rather as a subjective socially constructed concept based on characteristics that have acquired socially significant meaning (Williams, 1997). LaVeist (2000) has argued that race is a proxy for exposure to racism, which may be defined as the "institutional and individual practices that create and reinforce oppressive systems of race relations" (Krieger, 2003:195). Based on this conceptualization, some investigators hypothesize that 
race-associated differences in health outcomes are in fact partly due to the effects of racism (Schnittker \& McLeod, 2005).

Racism is an exclusionary process based on discourses and practices of inferiorization (Anthias, 1990). It is built on socially constructed physical and biological differences that are used to define groups and place them in a social hierarchy. Though racism is used in this dissertation as primarily a structural factor, it can have a direct impact on individuals' wellbeing. It may affect health by lowering self-esteem and increasing one's sense of helplessness (Williams et al., 2003). One of the ways in which racism manifests itself in today's society is through processes of discrimination against racial minorities.

Historically many racial and ethnic groups in the United States have faced severe discrimination or unfair treatment. As a result of discrimination many racial and ethnic group have been and continue to be denied civil, social, political, educational, and economic opportunities. Although many factors may contribute to such differences, their size and extent suggest that various forms of discriminatory treatment persist and serve to undercut the achievement of equal opportunity.

\section{Sexuality and Heterosexism}

Men who have sex with men face severe discrimination based on their sexual orientation. The literature conceptualizes sexual orientation in terms of sexual attraction, sexual behavior, sexual identity, or some combination of these dimensions (Wolitski, Valdiserri, \& Stall, 2008). Yet this grouping of men known as "men who have sex with men," includes a diversity of sexual attractions, identities, 
and behaviors, and is not synonymous with being gay, bisexual, or straight (Burks et al., 2011). An example would include a self-identified heterosexual man who partakes in same-sex sexual acts but not the identity of gay or bisexual. It is here that the population of MSM deviates from sexual orientation in part and is better conceptualized as a sexual minority group.

MSM share a historically marginalized social status relative to society's cultural norm of the exclusively heterosexual man who conforms to traditional gender roles and expectations. Put another way, MSM share the common status of "other" because they depart from heterosexual and gender norms (De Beauvoir, 1953). Their "otherness" is the basis for discrimination. Discrimination is enacted toward MSM through heterosexism, a socially constructed concept which is defined as "an ideological system that denies, denigrates, and stigmatizes any nonheterosexual form of behavior, identity, relationship, or community" (Herek, 1990:316). Research indicates widespread prevalence of discrimination experienced by MSM based on their sexual behavior (Mays \& Cochran, 2001; Ilan H Meyer, 1995; Schwartz \& Meyer, 2010). Furthermore, discrimination experienced by MSM is thought to challenge the development of healthy self-concepts (Hequembourg \& Brallier, 2009; Mays \& Cochran, 2001).

\section{Racial/Sexual Minorities and the Intersections of Racism and Heterosexism}

Both racial and heterosexist discrimination are predictors of poor health for individuals with membership to a racial or sexual minority group. However, in addition to being a member of one of these groups, individual and population health 
can be further diminished by the interactive effects resulting from membership to both groups. While many studies concentrate on the influence of a single identity in predicting health behavior and outcomes, fewer studies have examined the interactive effects of multiple identities (Barney, 2003). Both qualitative and quantitative studies suggest that experiences of multiple forms of discrimination may be associated with poor mental health outcomes among racial minority MSM. Racial minority MSM are likely to face racial discrimination both within mainstream white contexts and within LGBT communities, as well as facing heterosexist discrimination within the broad heteronormative environment and in racial ethnic minority communities (Díaz et al., 2001; Meyer, 1995, 2003; Yoshikawa et al., 2004).

\section{HIV Risk Behavior}

Since the beginning of the HIV epidemic, unprotected anal intercourse (UAI) has been a primary risk behavior for HIV infection among MSM with higher risk being associated with receptive intercourse compared to insertive intercourse (Koblin et al., 2006; Samuel et al., 1993; Seage et al., 1992; Vittinghoff et al., 1999). Explanations for and predictors of why MSM engage in UAI are well studied (Benotsch, Kalichman, \& Cage, 2002; Crepaz, Hart, \& Marks, 2004; Halkitis, Zade, Shrem, \& Marmor, 2004; Kelly, Hoffman, Rompa, \& Gray, 1998; Koblin, Perdue, \& Ren, 2003; Liau, Millett, \& Marks, 2006; Prestage, Jin, Grulich, de Wit, \& Zablotska, 2011; Remien, Wagner, Carballo-Dieguez, \& Dolezal, 1998; Rouwenhorst, Mallitt, \& Prestage, 2012; Suarez \& Miller, 2001; Sullivan, Drake, \& Sanchez, 2006; Vanable, Ostrow, McKiman, Taywaditep, \& Hope, 2000; Wolitski, 2005; Zablotska et al., 
2009). Some of the associated predictors of UAI among MSM include type of partner (Mitchell, 2013), alcohol and substance use (Bruce, Ramirez-Valles, \& Campbell, 2008; Choi et al., 2005; Hirshfield, Remien, Humberstone, Walavalkar, \& Chiasson, 2004; Mansergh et al., 2001; Parsons \& Kutnick, 2005; Purcell, Moss, Remien, Woods, \& Parsons, 2006; Reisner et al., 2010; Stall et al., 2001; Zablotska et al., 2009), "treatment optimism," the optimistic view of improved highly affective antiretroviral therapy (Crepaz et al., 2004; Halkitis et al., 2004; Kelly et al., 1998; Koblin et al., 2003; Remien et al., 1998; Sullivan et al., 2006; Vanable et al., 2000), seeking sex partners via the Internet (Benotsch et al., 2002; Liau et al., 2006; Zablotska et al., 2009), and risk-reduction strategies (Jin et al., 2007; Kippax et al., 1997; Parsons et al., 2005; Van De Ven et al., 2002; Varghese, Maher, Peterman, Branson, \& Steketee, 2002; Vittinghoff et al., 1999).

Given the complexity of HIV risk behavior among MSM and the fact that UAI is considered a primary risk factor for HIV transmission, this study conceptualizes HIV risk behavior as UAI with an understanding that the literature uses UAI alone or in combination with HIV status, sexual positioning, seroconcordant/serodiscordant partnerships, type of sexual partners, number of sexual partners, and monogamy to assess and understand HIV risk among MSM. The literature below highlights the relationships between discrimination, depression, and HIV risk behavior taking into account the various ways in which HIV risk behavior is operationalized, alcohol and substance use, and demographic factors to better understand the realities of risk among racial minority MSM and where appropriate Native MSM. 


\section{DISCRIMINATION AND HIV RISK BEHAVIOR}

In general the literature indicates experiences of discrimination are associated with increased UAI among MSM (Raymond, Chen, Stall, \& McFarland, 2011) and racial minority MSM specifically (Ayala et al., 2012; Diaz \& Ayala, 2001; Jarama, Kennamer, Poppen, Hendricks, \& Bradford, 2005; Mizuno et al., 2012). Qualitative investigations also support this association (Han, 2008; Stokes \& Peterson, 1998; Wilson \& Yoshikawa, 2004). However, the literature also indicates that the association is not uniform across studies. For instance, some studies find that both heterosexist and racial discrimination are associated with higher levels of UAI (Ayala et al., 2012; Díaz et al., 2001), whereas other studies indicate that only heterosexist discrimination is associated with UAI (Mizuno et al., 2012). Lastly, a few studies report no association between either racial or heterosexist discrimination and UAI (Hirokazu Yoshikawa et al., 2004).

The initial studies into this association were criticized for having small convenience samples, which "may be very different than the general population to which one wants to generalize" (Meyer, 2003, p685). Additionally, this research uses varying conceptualizations of sexual orientation, which compromises the validity of the studies as some studies recruited self-identified gay and bisexual men (Diaz, Ayala, \& Bein, 2004; Han, 2008), gay, bisexual, and transwomen (Bruce et al., 2008), MSM only (Ayala et al., 2012; Jarama et al., 2005; Mizuno et al., 2012; Stokes \& Peterson, 1998) and, in one case, gay and bisexual self-identified men and MSM (Hirokazu Yoshikawa et al., 2004).

More recent studies have been designed to address these methodological 
criticisms; the most well known is the Brothers y Hermanos study funded by the CDC. This study recruited 2235 Black and Latino MSM from Los Angeles County, New York City, and Philadelphia from May 2005 through April 2006 with the intention of identifying the sociocultural, psychosocial, and behavioral predictors of HIV infection among Black and Latino MSM (Ayala et al., 2012; Mizuno et al., 2012). This study has produced several research findings indicating that discrimination is associated with HIV sexual risk behavior among Black and Latino MSM (Ayala et al., 2012; Mizuno et al., 2012). Ayala and colleagues (2012) examined the impact of discrimination on whether participants had engaged in UAI within the past 3 months with male partners of serodiscordant (pairings in which HIV statuses differ between partners) or unknown HIV status. Using both mediation and path analysis techniques, their findings indicate that both heterosexist discrimination and racial discrimination were positively associated with increased participation in UAI with male sex partners of serodiscordant or unknown HIV status.

In another study stemming from the Brothers y Hermanos project, Mizuno and colleagues (2012) examined the associations of both heterosexist discrimination and racial discrimination with unprotected insertive and receptive anal intercourse with main and casual partners over the past 3 months. The findings of the multivariate logistic regression analyses indicated those experiencing heterosexist discrimination (but not racial discrimination) were at increased odds of reporting unprotected insertive anal intercourse with their main partner compared to those experiencing no discrimination. However, the experiences of discrimination were not significantly associated with reporting unprotected 
receptive anal intercourse with a main partner. Experiencing both heterosexism and racism were significantly associated with having unprotected receptive anal intercourse with a casual partner.

These studies stemming from the Brother y Hermanos project provide evidence supporting the association between discrimination and HIV risk behavior among racial minority MSM despite various operationalizations of discrimination. Ayala and colleagues (2012) created separate measures for racial discrimination and heterosexist discrimination and conducted their analysis using the measures independently, whereas Mizuno and colleagues (2012) created one measure of discrimination that incorporated both racial and heterosexist discrimination. Though drawn from the same data, these two studies did not use the same items to assess either racial or heterosexist discrimination. Despite these differences in operationalizing discrimination, both research projects found supporting evidence of the association between racial and heterosexist discrimination and UAI.

\section{The Role of HIV Status in HIV Risk Behavior}

These examples also assess HIV risk with an emphasis on UAI, however they differed on the aspects of exposure related risk factors such as HIV status and partner status. With regard to HIV status, it is important to contextualize HIV risk behavior with the fact that HIV-positive MSM have sex and sometimes they engage in unprotected or condomless sex. Though some HIV-positive MSM protect their sex partners from infection by eliminating or reducing UAI (Marks, Crepaz, Senterfitt, \& Janssen, 2005; Weinhardt, 2005; Wolitski, Bailey, O'Leary, Gomez, \& Parsons, 2003; 
Wolitski, Flores, O’Leary, Bimbi, \& Gomez, 2007), some HIV-positive MSM engage in UAI increasing the risk of HIV transmission to their sex partners (Crepaz \& Marks, 2002; Kalichman, 2000; Van Kesteren, Hospers, \& Kok, 2006). To reduce the possibility of transmitting HIV to others, HIV-positive MSM may practice a 'harm reduction' approach called serosorting. Serosorting is the behavior in which partnerships are understood to be seroconcordant (HIV statuses match among partners) or serodiscordant. Thus HIV-positive MSM engaging serosorting behavior may have unprotected sex only with other HIV-positive partners. Additionally, they may be less likely to have engage in sexual activity or more likely to use condoms with partners they perceive to be HIV-negative or of unknown status (Jin et al., 2007; Kippax et al., 1997; Parsons et al., 2005). In the exemplar from the Brothers y Hermanos study, Ayala and colleagues (2012) focused on UAI with serodiscordant partners. This operationalization of HIV risk assesses risk based on condom use and the knowledge that one's HIV status does not match that of his sexual partner(s). This measure is often considered "high" risk given that the partners have different HIV statuses and are not using condoms.

Another harm reduction that HIV-positive MSM may practice is called strategic positioning. Strategic positioning is an behavior in which HIV-positive MSM selectively engage in unprotected receptive anal intercourse (URAI) rather than unprotected insertive anal intercourse (UIAI) in serodiscordant partnerships given the per contact risk of transmission is lower when the infected partner is in the receptive position (Van De Ven et al., 2002; Varghese et al., 2002; Vittinghoff et al., 1999). In the exemplar from the Brothers y Hermanos study present above, 
Mizuro and colleagues (2012) focused on UAI with regard to sexual positioning among main and casual partners.

Other studies examining the relationship of HIV status and HIV risk behavior among racial minority MSM have mixed findings. Being HIV-negative compared to be HIV-positive was associated with greater risk behavior among Black MSM in one study (Myers, Javanbakht, Martinez, \& Obediah, 2003), but HIV status was unrelated to unprotected anal intercourse or condom use among Black MSM in a other studies (Centers for Disease Control and Prevention, 2004; Peterson et al., 1992). Similarly among Latino MSM, HIV status was not found to be associated with unprotected anal intercourse (Calabrese, Reisen, Zea, Poppen, \& Bianchi, 2012). A study of HIVpositive Latino men, found the strongest predictor of unprotected anal intercourse with the most recent partner was that the most recent partners was also HIVpositive, suggesting serosorting behavior among that population (Poppen, Reisen, Zea, Bianchi, \& Echeverry, 2004). In a study comparing serosorting behavior between Latino and Black MSM, Marks and colleagues (2010) found that Latino MSM are more likely to partake in serosorting behavior (HIV-negative participants engaging in UAI with a partner who was identified as also being HIV-negative) than Black MSM. The data are too limited to examine this association among Asian and Native MSM.

Though the literature has mixed findings about the association between HIV status and HIV risk behavior, the one consistent theme throughout the literature is that the motivation for using condoms is different for HIV-positive MSM compared to HIV-negative MSM. That is to say that those who are HIV-negative use condoms to 
protect themselves as a means of personal responsibility, whereas those that are HIV-positive use condoms to protect others as a means of social or collective responsibility (Mansergh et al., 2010).

\section{The Role of Partner Status in HIV Risk Behavior}

In addition to HIV status and harm reduction approaches, HIV risk behavior also takes into account partner status. Partner status is understood as a relative risk that MSM may engage in UAI depending on whether their sex partner is a main partner (a person with which you share a strong emotional connection with) or a casual partner (often considered a sex partner that is not your main partner). MSM may have only a casual partner(s), only a main partner(s), or may have both casual and main partners. Having a main partner is associated with HIV-protective and risk behaviors among MSM.

With respect to protective behaviors, the literature highlights that MSM engage in UAI with their main partner as a way to strengthen their commitment and overall relationship satisfaction (Davidovich, de Wit, \& Stroebe, 2006; De Vroome, Stroebe, Sandfort, de Wit, \& Van Griensven, 2000; McLean, Boulton, \& Brookes, 1994; McNeal, 1997). Within the partnership, UAI can be part of a sexual agreement (Mitchell, 2014) and / or an act demonstrating their love, intimacy, and trust toward one another (Blais, 2006; Davidovich, de Wit, \& Stroebe, 2004; De Vroome et al., 2000; McLean et al., 1994; McNeal, 1997). However, the literature also provides some evidence that MSM engage in UAI with main partners for reasons that focus less on relationship commitment, love, and intimacy. Studies have reported that 
MSM engage in UAI with their main partners because "they perceive to know them 'well' and have previously had anal intercourse with them" (Mitchell, 2013:1558). Another type of protective behavior that MSM may engage is monogamy. Monogamous relationships are relationships in which partners agree to and only engage in sexual activities with each other and no one else. Monogamy is thought to influence sexual activity and reduce condom use among MSM. That is to say that men in monogamous relationships who have seroconcordant HIV statuses can engage in UAI with less risk (or no perceived risk) because they are not engaging in UAI with anyone other than their partner whose HIV status matches his own. This phenomenon is difficult to assess as monogamy status is mutually agreed upon, but may not be a reality in the event that one of the partners has agreed to be monogamous but in fact has more than one concurrent sex partner (Crepaz et al., 2000).

Given the difficulty in assessing perceived monogamy (based on an agreement of both partners) versus actual monogamy (based on behavior of both partners), HIV transmission can occur in monogamous relationships. HIV transmission can occur in perceived monogamy situations in which one partner acquires HIV from a sexual partner outside of the monogamous relationship. Additionally, HIV transmission can occur in actual monogamous relationships in which HIV seroconversion occurs in one partner (thought to be HIV negative) after monogamy was agreed upon and condom use becomes less consistent. Epidemiological studies indicate that engaging in UAI with a main partner is a significant source of HIV infection among MSM (Davidovich et al., 2001; Moreau- 
Gruet, Jeannin, Dubois-Arber, \& Spencer, 2001; Sullivan, Salazar, Buchbinder, \& Sanchez, 2009) as MSM engage in higher rates of UAI with their main partners and thereby increase their risk of transmitting or acquiring HIV (Ekstrand, Stall, Paul, Osmond, \& Coates, 1999; Elford, Bolding, Maguire, \& Sherr, 1999; Hoff et al., 2009), highlighting the importance of both perceived and actual monogamy in the transmission of HIV. One study indicated that approximately 68\% of MSM acquire HIV while in a same-sex relationship (Sullivan et al., 2009).

There is some evidence to suggest that racial minority MSM engage in protective behaviors and risk behaviors with main versus causal partners. For example, Lauby and colleagues (2008) found that HIV-positive Black MSM were less likely to engage in UAI with their HIV-negative or HIV-unknown main partners than with their HIV-negative or HIV-unknown casual partners, suggesting a protective behavior for main partners. However, other studies among racial minority MSM suggest risk behavior. For example, one study found that partnered Black MSM are more likely to engage in UAI with their main partner than their causal partner(s) (Hart \& Peterson, 2004). Additionally, Calabrese and colleagues (2012) found that among immigrant Latino MSM, those that were partnered were more likely to engage in unprotected receptive anal intercourse compared to those without a partner. However, these studies highlight the reasons for, and factors associated with, UAI among MSM are complex. UAI with a main partner may be contingent upon HIV status.

To date the literature focuses only on the experiences of Asian, Black, and Latino MSM as there have been no investigations examining the association between 
discrimination and HIV risk behavior among Native MSM. The literature overall and the examples presented above suggest that there is association between racial and heterosexist discrimination and HIV risk behavior across various methods of analyzing discrimination as well as varying measures of HIV risk behavior. The evidence suggests that racial and heterosexist discrimination will be associated with HIV risk behavior for Native MSM.

Though there is evidence to suggest that discrimination is associated with HIV risk behavior, the mechanisms that explain this association are unclear. The most prominent mechanism is depression. Specifically, the experience of racial discrimination may induce psychological distress that may adversely affect mental health status (Williams \& Collins, 1995). In particular, Williams and colleagues (2003) suggested that stress from discrimination produces a negative emotional reaction including "depression, which in turn can have direct effects on biological processes or patterns of behavior that affect disease risk" (p. 205). In addition to race, there is a growing body of research examining the link between heterosexist discrimination and depression (Aneshensel, 1992; Turner \& Lloyd, 1999).

\section{DISCRIMINATION AND MENTAL HEALTH}

A growing body of research on mental health outcomes suggests that certain social statuses, such as race and sexuality, influence the likelihood of exposure to deleterious experiences (Aneshensel, 1992; Turner \& Lloyd, 1999). The literature documents the experiences of discrimination and the disparities in mental health outcomes among racial minority MSM. Studies assess the effects of racial 
discrimination (Choi, Paul, Ayala, Boylan, \& Gregorich, 2013; Díaz et al., 2001; Hirokazu Yoshikawa et al., 2004), heterosexist discrimination (Choi et al., 2013; Díaz et al., 2001; Meyer, 1995; Yoshikawa et al., 2004), immigrant based discrimination (Chae \& Yoshikawa, 2008), and in some cases combinations of various types of discrimination on mental health outcomes (Díaz et al., 2001). Furthermore, this literature contains a wide range of mental health outcomes, which includes depression (Chae \& Yoshikawa, 2008; Choi et al., 2013; Herek, Gillis, \& Cogan, 1999; Mays \& Cochran, 2001; Hirokazu Yoshikawa et al., 2004), anxiety (Choi et al., 2013; Mays \& Cochran, 2001), panic disorder (Mays \& Cochran, 2001), and psychological distress (Chen \& Tryon, 2012; Díaz et al., 2001). However, this literature uses varying types and sources of measures to assess both discrimination and depression which may limit our understanding of the association between discrimination and mental health and more specifically the association between racial and heterosexist discrimination and depression among racial minority MSM.

Despite these inconsistencies in measures and outcomes, there are a few studies that indicate experiences of discrimination are associated with elevated rates of depression among racial minority MSM (Choi et al., 2013; Díaz et al., 2001; Hirokazu Yoshikawa et al., 2004). These studies show that racial minority MSM experience elevated rates of depression, even after controlling for age, employment status, educational attainment, HIV status, income and self-reported sexual orientation. Although many determinants are associated with elevated rates of depression among racial minority MSM are unknown, researchers have identified racial and heterosexist discrimination as major contributors. For example, Diaz and 
colleagues (2001) examined psychological distress and its association with heterosexist, racial, and immigrant-based discrimination among 912 urban dwelling Latino gay and bisexual men. Their findings indicate that experiences of heterosexist, racial, and immigrant-based discrimination significantly predicted symptoms of psychological distress. It is important to note that psychological distress in this study was assessed using measures for anxiety, depression, and suicidal ideation.

In another study, Yoshikawa and colleagues (2004) examined the influence of experiences of discrimination on depressive symptoms and HIV risk among a sample of 192 Asian and Pacific Islander gay men. Racial and heterosexist discrimination were assessed using scales adapted from the work of Rafael Diaz and colleagues (2001). Depressive symptoms were assessed using the 20-item Center for Epidemiological Studies Depression scale (CES-D). The findings indicate that only racial discrimination proved to be associated with depressive symptoms among this sample. Though similar to the findings of Diaz and colleagues work among Latino MSM, the findings also suggested for the first time that the experiences of discrimination matter differently for different racial minority MSM populations. Later studies suggest that Asian MSM experience high levels of depressive symptoms as a result of racial discrimination within the mainstream gay community (Chae \& Yoshikawa, 2008).

Lastly, Choi and colleagues (2013) set out to examine the association between experiences of discrimination on depressive symptoms among a sample consisting of 393 Asian MSM, 403 Black MSM, and 400 Latino MSM and how this 
association varied by racial group. Experiences of racial discrimination and heterosexist discrimination were assessed by source (general community, gay community, heterosexual friends, and family members). Depressive symptoms were assessed using the 20 -item CES-D. Findings from the multivariate regression analysis state that experiences of racial discrimination from the general community and heterosexist discrimination among heterosexual friends were associated with elevated depressive symptoms among the sample. This study also reported that heterosexist discrimination from heterosexual friends but not family is associated with depressive symptoms, regardless of racial group. Taken together, these findings indicate that discrimination type and source have differential impact on depressive symptoms depending on racial group membership.

These examples support the association between discrimination and depression among racial minority MSM. The discrimination measures in these studies were similar in that Yoshikawa and colleagues (2004) adapted their questions from the study conducted by Diaz and colleagues (2001). Using similar measures allowed for a comparison between racial minority groups. Despite using similar measures, the results between the groups were different, which suggests that discrimination may matter differentially for different racial minority groups. To address the methodological limitations of comparing different samples using similar measures, Choi and colleagues (2013), assessed this association between discrimination and depression across multiple racial minority MSM comprised in one overall sample. The findings implicate the types and sources of discrimination matter differently for each racial minority group. 
In addition to race and ethnicity among MSM, evidence found in the literature supports the relationship between socioeconomic status and depressive symptoms among MSM. Among Latino MSM, Diaz and colleagues (2001) found a positive associations between poverty and increased psychiatric distress. Using the CES-D to assess depressive symptoms, De Santis and colleagues (2008) also found that both lower income and lover levels of education were associated with depressive symptoms among Latino MSM. However, this association also suggests that socioeconomic status differentially influences depressive symptoms by racial group. Yoshikawa and colleagues (2004) found no association between income and depressive symptoms among Asian MSM. Furthermore, though Choi and colleagues (2013) found that having less than a high school degree was associated with increased levels of depressive symptoms in bivariate analysis across groups of racial minority MSM, the association was explained away in multivariate analysis.

Despite, various conceptualizations of depression, the literature overall suggests that there is association between racial and heterosexist discrimination and depressive symptoms among racial minority MSM. The study conducted by Diaz and colleagues (2001) used an adapted measure of 5 items to assess psychological distress in which depression is only one of several factors and both Yoshikawa and colleagues (2004) and Choi and colleagues (2013) used the CES-D scale to assess the severity of depressive symptoms, suggest some consistent use in measures regarding depressive symptoms. Additionally, the research indicates that the association between discrimination and depression plays out differentially based on racial minority group membership. The most recent research has found 
that the sources and types of discrimination differentially impact depression among different racial groups.

\section{DEPRESSION AND HIV RISK BEHAVIOR}

Empirical studies examining the association between depression and HIV risk behavior have yielded inconsistent results (Crepaz \& Marks, 2001). Some of the initial studies conclude that a negative association exists between depression and HIV risk behaviors suggesting that depressed people would use greater caution and ultimately take less risk (Frijida, 1988). However, other studies argued in favor of a positive association between depression and sexual risk taking (Marks, Bingham, \& Duval, 1998; Strathdee et al., 1998). In 2003, Rogers and colleagues suggested that varying types of depressive disorders (such as major depression, clinical depression, and depressive symptoms) needed to be accounted for as a means of addressing the inconsistencies in previous studies. Since then, a growing number of studies indicate that depression is associated with HIV-risk behavior among MSM (Rogers et al., 2003; Stall et al., 2003). However, the more recent literature is inconsistent with studies showing support of a positive relationship among Black MSM (Crawford, Allison, Zamboni, \& Soto, 2002; Myers et al., 2003; Reisner et al., 2009), despite some evidence to suggest that there is simply no association between depression and HIV risk behaviors among Asian and Pacific Islander MSM (Yoshikawa et al., 2004). 


\section{Depression and HIV Risk among Black MSM}

Among Black MSM, the literature indicates a positive association between depressive symptoms and HIV risk behaviors (Crawford et al., 2002; Myers et al., 2003; Reisner et al., 2009). For example, Reisner and colleagues (2009) examined the associations of depressive symptoms and behavioral HIV risk factors among a sample of 197 Black MSM. Depressive symptoms were assessed using the 20-item CES-D. The HIV risk factors assessed with respect to anal intercourse were condom use, serodiscordancy, and partner type. Multivariate logistic regression modeling indicated that depressive symptoms were positively associated with serodiscordant UAI with a causal partner, which supports previous studies claiming the positive association between depressive symptoms and HIV risk behavior.

Similarly, Myers and colleagues (2003) examined the associations of psychosocial factors and HIV risk behavior among a sample of 502 mixed HIV serostatus Black men, half of whom identified as MSM or MSMW (men who have sex with men and women). The study assessed depression using the SCL 90-R, despite the fact that the CES-D was also used. Given that both scales are highly correlated with each other, Myers and colleagues selected to use the SCL 90-R for the analysis. Risky sexual behavior was assessed using a sexual risk index designed specifically for this study comprised of a series of questions about respondents' sexual history and current lifestyle, especially sexual behaviors that placed them at high-risk for sexually transmitted infections and HIV. The index included the number of sexual partners, sex while incarcerated, condom use in monogamous relationships versus condom use with multiple partners, exchange of sex for money or drugs, and sex 
under the influence of alcohol or drugs. Poisson regression analysis indicated that both Black MSM and MSMW participate in more unsafe or risky sexual behavior than their heterosexual counterparts and more specifically:

"African American men who were most likely to engage in more high-risk sexual behaviors were those who had a bisexual lifestyle, were HIV-negative, experienced greater psychological distress, had limited resources for coping (i.e., were less educated and unemployed), had established sexual high-risk habits and preferences (i.e., older age), and those who were more socially isolated (i.e., had less social support)" (p. 76).

\section{Depression and HIV Risk among Asian and Pacific Islander MSM}

There is a dearth of information regarding the association between depression and HIV risk behavior among Asian and Pacific Islander MSM. Of the recent literature, the focus is devoted to ethnic and sexual identity development among Asian (and where appropriate Pacific Islander) populations (Chae \& Yoshikawa, 2008; Wilson \& Yoshikawa, 2004; Yoshikawa et al., 2004). Yoshikawa and colleagues (2004) measured depression using the CES-D among 192 Asian and Pacific Islander MSM and measured HIV risk behavior using self-reported UAI during the past three months. Forty-five percent of the sample reported risk of clinical depression, which is consistent with other samples of Asian MSM (Chae \& Yoshikawa, 2008). UAI with main and causal partners (19\% and $17 \%$ respectively) is also consistent with other Asian MSM samples (Chae \& Yoshikawa). However, the logistic regression analysis indicated no association between depression and UAI within the sample. Given this was not the primary question of this study, no explanation was provided for the lack of the association. 
The inconsistent findings in the literature could indicate that the association between depression and HIV risk behaviors is dependent on other factors that are not assessed in these studies. The additional factors could be related to the individual characteristics of participants in the research and their partner, such as socioeconomic status, as well as the dynamics of the dyad and other individual characteristics that were not captured in these studies (Poundstone et al., 2004). Furthermore, the inconsistent results could be due to other social and cultural determinants that are not measured in these studies such as other forms of discrimination or stigma, sexual network dynamics, public health policy, and access to prevention and health services. The literature overall and the examples presented above suggest inconsistent findings in the association between depressive symptoms and HIV risk behavior among racial minority MSM. However, there is some evidence to suggest a positive association among Black MSM, which further demonstrates that different racial minority groups respond to and behave differently than others.

\section{The Role of Alcohol and Substance Use}

Two of the main factors that are suggested to influence the association between depression and HIV risk behaviors are alcohol and substance use. A growing number of studies that describe the associations between depression, alcohol use, substance use, and HIV risk behavior among MSM (Bruce et al., 2008; Choi et al., 2005; Hirshfield, Remien, Humberstone, Walavalkar, \& Chiasson, 2004; Parsons \& Kutnick, 2005; Purcell, Moss, Remien, Woods, \& Parsons, 2006; Reisner et 
al., 2010; Stall et al., 2001). The most prominent studies that investigate all of these associations stem from the Urban Men's Health Study, a household-based sample of MSM who reside in some of America's largest cities who were interviewed in the late 1990s (Stall et al., 2001). The Urban Men's Health Study investigated a wide set of health conditions including depression, substance use, and HIV risk among others. The various research findings from this study indicate these conditions are interconnected among MSM. For example, Stall and colleagues (2003) reported that many of these conditions were independently associated with each other in multivariate models. Their findings indicate that depression is independently associated with polysubstance use (the use of multiple drugs over a period of time) and partner violence; that polysubstance use is independently associated with depression and partner violence; and that partner violence is independently associated with depression and polysubstance use. Furthermore each of these associations are positive and men who had experienced elevated measures in any one of these conditions had also reported engaging in HIV risk behaviors. Unfortunately, causal conclusions about these associations cannot be made given that the data are cross-sectional. All of this is to say that the associations between depression, substance use, and HIV risk behaviors are not well understood among MSM and more specifically among racial minority MSM.

However, there are a few studies that attempt to examine the association between depression and alcohol among racial minority MSM (Reisner et al., 2010; Stall et al., 2001). Among a sample of 197 of Black MSM, Reisner and colleagues (2010) examined the association of depression and problematic alcohol use. 
Depression was assessed using the CES-D. The CAGE questionnaire, a four-item validated clinical screening instrument for alcoholism, was used to assess problematic alcohol use. The logistic regression analysis indicated that participants with depressive symptoms had $40 \%$ higher odds of having problematic alcohol use compared to those without depressive symptoms.

There is a growing body of literature assessing the associations of alcohol and substance use with HIV risk behavior among MSM (Bruce et al., 2008; Hirshfield et al., 2004; Mizuno et al., 2012; J. Parsons \& Kutnick, 2005; Purcell et al., 2006). For example, a national sample of $3000 \mathrm{MSM}$ found that both alcohol and substance use were independently associated with UAI (Hirshfield et al., 2004). Studies also show that racial minority MSM have high rates of alcohol and substance use and that use is associated with UAI (Bruce et al., 2008; Mizuno et al., 2012; Reisner et al., 2010). In a study of 643 Latino MSM from Chicago and San Francisco, Bruce and colleagues (2008) examined the association of discrimination and unprotected receptive anal intercourse and the extent to which alcohol and substance use impacted the association. Alcohol use was assessed using a frequency-quantity index (AQFI) creating 4 categories of drinkers (abstainers, light drinkers, moderate drinkers, and heavy drinkers). Participants were also asked whether they had used the following substances in the past six months: marijuana, cocaine, amphetamines, crack, heroin, ecstasy, GHB, special K, tranquilizers, or sedatives. Based on sample distributions, participants were categorized into three groups: no drug used, one drug used, or multiple drugs used. Lastly, sex under the influence of alcohol and sex under the influence of drugs were assessed separately using a frequency scale of 
never, once or twice, and more than twice. All of the substance use variables served as intermediary variables in the analysis. The results of the structural equation modeling indicate strong associations between substance use and unprotected receptive anal intercourse. Furthermore alcohol and multiple substance use were associated with sex under the influence, which was associated with URAI. All this is to say that there are associations between alcohol use, substance use, and HIV risk behavior. However, it is important to note that like many other studies, the data in this study are cross-sectional and thereby restrict the ability to discuss causality.

Data from the Brother y Hermanos project also suggest that alcohol use, specifically binge drinking, is associated with HIV risk behavior. Mizuno and colleagues (2012) assessed binge drinking as a dichotomized variable on whether binge drinking occurred at least once in the past 3 months. Participants also reported whether they had used any of the following drugs in the past 3 months: methamphetamine, cocaine, crack, ecstasy, GHB, ketamine, or amyl nitrite (poppers). Unlike previous studies, drug use was not associated with HIV risk behavior.

The literature, despite its small size and mixed findings, indicates that alcohol and substance use are important factors when examining the association between depression and HIV risk behaviors among racial minority MSM. Furthermore, the literature implicates substance use as both a maladaptive coping strategy in and of itself, and as a mediating variable in the association between depressive symptoms and HIV risk behavior among racial minority MSM. However, it is important to note that these studies use cross sectional data limiting findings to 
associations and not allowing causal inferences to the direction of the associations.

\section{INFLUENCES OF CULTURAL DETERMINANTS}

The previous sections addressed the associations between discrimination, depression, and HIV risk behavior. This section reviews the research around cultural determinants of health, specifically social support, cultural identity, and community participation, and how they act as protective factors against depression and HIV risk behavior among racial minority MSM that are experiencing discrimination.

\section{Overview of Cultural Determinants}

Though small in scope, the cultural determinants literature has focused on factors that have been shown to improve health and are often discussed as being "protective" factors. For example, specific conversations with family and friends about discrimination among Asian and Pacific Islander MSM, were found to protect against UAI (Yoshikawa et al., 2004). Among a sample of gay and bisexual identified men, family acceptance, social and sexual satisfaction, and participation in social activism were found to protect against psychological distress (Diaz et al., 2001). All of these factors predicted better connections within either their racial or sexual communities resulting in elevated self-esteem. Furthermore, elevated self-esteem was found to be associated with decreased levels of depression and lower HIV risk behavior. As such the following is a review of the cultural determinants (social 
support, racial and sexual identity, and racial and sexual community participation) that protect racial minority MSM against the harms of discrimination.

\section{Social Support}

The importance of social support on health and heath behaviors has become increasingly clear. Both seeking and receiving help from other people is a form of coping that may protect people from negative consequences of stressful situations like discrimination (Sherbourne \& Stewart, 1991). The literature on social support indicates that increased levels of social support buffers the impact of depression related to discrimination (Meyer, 2003; Peterson, Folkman, \& Bakeman, 1996; Strathdee et al., 1998; Szymanski, 2009) and reduces HIV risk behaviors among racial minority MSM (Ayala et al., 2012; Vu, Choi, \& Do, 2011). Findings from the Brothers Y Hermanos project indicate that social support diminished the impact of discrimination on depression and HIV risk behaviors (Ayala et al., 2012).

\section{Racial and Sexual Identity}

Racial and sexual group identity may protect against the influence of discrimination on health. Research has shown that greater racial group identification and racial centrality diminish the effect of discrimination on depression among Black (Branscombe, Schmitt, \& Harvey, 1999; Sellers, Caldwell, Schmeelk-Cone, \& Zimmerman, 2003; Sellers \& Shelton, 2003) Latino (Romero \& Roberts, 2003), Asian (Chae \& Yoshikawa, 2008; Han, 2008), and Native populations (Walters, 1999). Additionally, research has shown that greater sexual group 
identification and centrality diminish the effect of discrimination on depression among sexual minorities (Meyer, 1995; Raymond et al., 2011). Furthermore, research has shown that identity of sexual minorities that belong to racial minority groups diminish the effect of discrimination on depression. Specifically, a study of Asian gay men found that endorsing positive evaluations of their own group (Asian gay men) protected against depression and diminished the influence of perceived devaluation by others on depression and HIV risk behavior (Chae \& Yoshikawa, 2008).

However, there is debate in the literature regarding the effects of developing both strong identities with one's racial group and sexual group as they these identities may have opposing interests. Using qualitative methods with a sample of 76 Black gay and bisexual men, Stokes and Peterson (1998) found that belonging to both racial and sexual minority groups comes with increased stress and participants "mentioned that being gay and Black was difficult because of the double minority status" (289). Participants were "told that they can't be a strong black man and be gay" and that "adopting an identity as gay or bisexual necessarily involved some degree of abdicating their identity as African Americans" (Stokes \& Peterson, 1998:289). Stokes and Peterson (1998) also indicated that persons belonging to multiple minority groups are also thought to have fewer resources to cope with negative life events, placing them at risk for depression and HIV risk. Specifically,

"Respondents mentioned that lack of acceptance from either the Black or gay communities leads to anger, frustration, and hopelessness - feelings that are not likely to increase the probability of protecting oneself against HIV and AIDS. The lack of a sense of community among Black MSM also decreases the chances that norms of safer sex will be communicated and established" (Stokes 
\& Peterson, 1998:289).

The quantitative research suggests that having strong racial and sexual group identities serve as protective factors reducing depression and HIV risk behaviors. Yet, the findings from qualitative research suggest that racial minority MSM may have to negotiate both a racial and sexual group identity formation (Chae \& Yoshikawa, 2008). This negotiation is also seen within the literature on acculturation and community participation.

\section{Racial and Sexual Community Participation}

The literature on the association of community participation and HIV risk behavior conclude that racial minority MSM who were more integrated into the gay community are less likely to engage in unprotected sex. For example, Ratti, Bakeman and Peterson (2000) show that Asian MSM who were less engaged with the gay community were more likely to report high risk sexual behaviors. Similarly, in another study that used subscription to gay publications as a proxy for community participation within the LGBT community, Seibt and colleagues (1995), found that racial minority MSM with more subscriptions to gay publications were less likely to engage in high risk sexual behavior. Lastly, Chng and Geliga-Vargas (2000) argued that racial minority MSM who isolate themselves from the gay community and gayidentified social networks also isolated themselves away from environment and networks that expose them to safe sex messages and discussions of HIV resulting in increased HIV risk behaviors. This research provides some evidence that community participation, particularly in the gay community, has been 
conceptualized as having a protective or buffering effect regarding HIV risk behavior.

However, the literature on the association of community participation of racial minority MSM into the mainstream white community and HIV risk behavior indicate that those who select to engage with their own racial minority group are more likely to engage in HIV risk behaviors. Chng and Geliga-Vargas (2000) point out that not only are racial minority MSM not engaging with the gay community they are simultaneously engaging in their respective racial or ethnic communities and therefore not engaging in the mainstream white community. As a result Chng and Geliga-Vargas conclude that increased participation in unprotected sex is due to not engaging with the mainstream white community. The authors also speak about racial minority phenomena of the down low, machismo, and model minority. However, Han (2007) argues the racial minority MSM need to maintain connection to their racial and ethnic communities as a means of maintain strong racial and ethnic identities and improved self-esteem.

The debate in the literature makes reference to the racial and sexual identity of two-spirit men. Of the small amount of literature that exists, it is posited that twospirit men do not need to acculturate into mainstream white communities but rather enculturate in native communities. Enculturation is understood as a process of community engagement that does not force two-spirit men to 'fit' into white definitions of 'gay' or 'Native'. Enculturation reclaims a long cultural practice of valuing a third gender that is not rigidly linked to the European definitions of 'man' or 'woman' nor 'gay' or 'straight' (Brown, 1997). The process of enculturation 
bolsters the self-esteem among two-spirit men and is thought to serve as a protective factor against depression and HIV risk behaviors. In fact, Walters (1999) demonstrates that enculturation into Native communities is critical for decreased depression and adverse health behaviors among Natives.

Despite being one of the larger gaps, the literature regarding the cultural determinants of racial minority MSM indicates that social support, racial and sexual identity, and racial and sexual community participation protect against the impact of discrimination on depression and HIV risk behaviors. There is a dearth of studies that specifically focuses on these effects within racial minority MSM and fewer that focus on these effects among Native MSM. There is a need for more investigations regarding cultural determinants that explore different conceptualizations, types, frequency and place of social support, identity, and community participation.

\section{OTHER ASSOCIATIONS AND HIV RISK BEHAVIOR}

It is important to address the demographic factors and their associations with HIV risk behavior at the individual and encounter level before discussing the associations of discrimination, depression, and HIV risk behavior at the structural level. Variables that describe characteristics of individuals are demographic factors. Demographic factors include age, HIV status, partner status, and socioeconomic status (education, employment, and income). HIV status and partner status have been discussed previous. The following is a review of the literature regarding the associations of age and HIV risk behavior as well as socioeconomic status and HIV risk behavior. 
Age

Considerable attention has been given to the association between age and HIV risk behavior, with specific attention to the "young age effect" or the fact that youth tend to take more risk and have higher rates of HIV infection than their older counterparts (Mansergh \& Marks, 1998). According to Mansergh and Marks (1998:1119), this research regarding this association yields inconsistent results due to varying "research designs, outcome measures, operationalizations of the age variable, and other methodological dimensions." Furthermore, in their review of the literature, Mansergh and Marks (1998) found that 13 of the 15 studies conducted in the United States provided evidence of increased HIV risk behavior among younger MSM compared to older MSM.

With respect to the young age effect among racial minority MSM, the results vary by racial group. Studies examining Black MSM consistently report no young age effect, whereas studies regarding Latino MSM consistently report a young age effect. Myers and colleagues (2003) found that older Black MSM engaged in more sexual risk than younger Black MSM, but other studies found no association between age and HIV risk behavior among Black MSM (Hart \& Peterson, 2004; Millett, Peterson, Wolitski, \& Stall, 2006; Peterson et al., 1992). Studies consistently report that younger Latino MSM engage in HIV risk behaviors, even among immigrants (Calabrese et al., 2012) and those that are HIV-positive (Poppen et al., 2004). Calabrese and colleagues (2012) attribute increased HIV risk behavior among young Latino MSM is in part due to emotional aspects of pleasure in sexual decision- 
making as there is a greater desire for physical sensation and partner intimacy among young Latino MSM. Additionally, the young age effect among Latino MSM is attributed to engaging in difficult sexual situations (i.e. sexual activity aimed to alleviate mental distress, sexual activity within relationships of unequal power, and sexual activity while under the influence of drugs and alcohol)(Diaz et al., 2004; Wilson, Díaz, Yoshikawa, \& Shrout, 2009).

Despite the lack of studies examining the young age effect among Asian MSM, the few studies of young Asian MSM indicate comparable rates of HIV risk behavior compared to samples of young Latino and White MSM. For example, Ruiz and colleagues (1998) found in a sample of 17-25 year old MSM from four major cities that Asian MSM had comparable rates of UAI compared to White MSM of the same age (39\% vs. $36 \%)$, but had higher rates of UAI compared to Black MSM of the same age (39\% vs. 30\%). In a study conducted in 13 cities across the U.S., Peterson and colleagues (2001) found similar findings in that Asian MSM ages 15-25 had a higher rate of UAI compared to White and Black MSM of the same age. Lastly, using data from the Young Men's Study, Bingham and colleagues (2003) found that Asian MSM had higher rates of UAI and URAI compared to Black MSM but lower rates compared to Latino MSM.

The limited data available regarding young Natives and young Native MSM demonstrates inconsistent condom use among youth and additional studies are needed to examine the young age effect with respect to HIV risk behavior among these populations. However, in a study of over 13,000 Native youth, only $49 \%$ of males reported using condoms during sex (Blum, Harmon, Harris, Bergeisen, \& 
Resnick, 1992). With respect to Native MSM, Simoni and colleagues (2006) reported no significant difference in condom use between Native MSM to Native men that only have sex with women. This finding was attributed to the high amounts of condomless sex reported by both groups. Among sexually active respondents overall, $72 \%$ reported that they had engaged in sex without a condom at least once and $68 \%$ reported that they did not use a condom during their most recent sexual encounter (Simoni et al., 2006).

Despite having only a few studies examining the effect of age on HIV risk behavior and the variations in designs between studies; the findings among racial minority MSM indicate that HIV risk behavior is associated with age for Latino MSM (and possibly Asian MSM), but not for Black MSM. The limited data regarding young Native MSM indicate inconsistent condom use overall and high rates of unprotected sex, which suggesting the possibility of a young age effect, though further investigations are necessary.

\section{Socioeconomic Status}

Socioeconomic status (SES) is an "individual's position in a system of social stratification that differentially allocates the major resources enabling people to achieve desired goals and health" (House \& Williams, 2003:91). Education, employment, and income are the traditional measures used to assess SES. Though the associations between these measures and HIV infection have been well studied (Farmer 1999, 2005, Zierler and Krieger 1998), the associations between these measures and HIV risk behavior among MSM are less well known. The literature 
below highlights exemplars of this line of research by each measure and the various ways in which they are operationalized to better understand the realities of risk among MSM.

\section{Educational Attainment}

The literature regarding the association between educational attainment and HIV risk behavior among MSM has mixed findings. The literature states that among samples of HIV-negative gay and bisexual men (Appleby et al., 2005; Strathdee et al., 1998), young gay and bisexual men (Janssen, De Wit, Stroebe, \& Griensven, 2000), a national online sample of MSM (Hirshfield et al., 2004), and a national sample for an intervention trial (Colfax et al., 2004), there is an association between educational attainment and HIV risk behavior. Conversely, among samples of Black MSM (Hart \& Peterson, 2004), Latino MSM (Nakamura \& Zea, 2010), and gay and bisexual men of color (Chng \& Geliga-Vargas, 2000), the literature states that there is not an association between educational attainment and HIV risk behavior.

Despite the differences in the operationalization of educational attainment, the inconsistent findings are attributed to the fact that most same-sex sexual attraction and sexual minority identity begin to form long after one's education is under way. As such, the association between educational attainment and HIV risk behavior will naturally be less strong (Wolitski et al., 2008). Furthermore, some sexual minority men are able to hide their sexual attractions, behaviors, and identities, and as such they are able to start on equal ground with their counterparts (Wolitski et al., 2008). Lastly, it is important to note that among the literature cited 
above, the samples indicating an association included MSM from all racial backgrounds and as such the association of educational attainment and HIV risk maybe influenced by the effects of race on educational attainment, which further explains why there is no association in samples consisting of only racial minority MSM.

\section{Employment}

The literature among the association between employment and HIV risk among racial minority MSM, has mixed findings (Allegretto \& Arthur, 2001; Black, Gates, Sanders, \& Taylor, 2000; Hart \& Peterson, 2004; Myers et al., 2003; Tebaldi \& Elmslie, 2006). Among a samples of Black MSM, Hart and Peterson (2004) found the employment status was not associated with HIV risk behavior, whereas Myers and colleagues (2003), found that those who were unemployed had increased HIV risk behavior. This literature is contextualized by the fact that that men living with a same-sex partner work fewer hours and are more likely to work part time compared to married and unmarried heterosexual men (Tebaldi \& Elmslie, 2006). Furthermore, MSM that live with a same-sex partner who become unemployed are also more likely to remain unemployed compared to their heterosexual peers.

\section{Income}

The only measure of SES that is consistently associated with HIV risk behavior is income. The literature on MSM indicates that as income increases the rates of UAI (Hirshfield et al., 2004), serodiscordant UAI (Colfax et al., 2004), and 
URAI (Appleby et al., 2005) decrease. The association remains consistent among racial minority MSM, despite variation in the operationalization of income. Studies have found that among Black MSM, those with lower income engaged in more HIV risk behavior than those at higher income levels (Myers et al., 2003; Peterson et al., 1992). Similarly among Latino MSM, Nakamura and Zea (2010) found those with higher income levels reported lower levels of UAI compared to those at lower income levels. Lastly among Asian and Pacific Islander gay men, Yoshikawa and colleagues (2004) found that as income increased the rates of UAI with a main partner decreased.

Despite the consistent association between income and HIV risk behavior among MSM, the mechanisms for understanding this association remain largely understudied. Though it is widely known that MSM have similar or sometimes higher reported levels of educational attainment, most studies of MSM indicate that they make less than their heterosexual counterparts (Allegretto \& Arthur, 2001; Black et al., 2000; Tebaldi \& Elmslie, 2006). This disparity is in part due to differences in wages and differences and workforce participation. However, this same trend in differential income is found among the MSM community within the context of HIV status. Myers and colleagues (2003) found that HIV-positive MSM had similar levels of education compared to HIV-negative MSM, but HIV-positive MSM were significantly more likely to be working part-time or unemployed and as a result they earned significantly lower incomes than those that were HIV-negative.

There are several studies that support an association between socioeconomic indicators and HIV risk among racial minority MSM (Appleby et al., 2005; Colfax et 
al., 2004; Hirshfield et al., 2004; Myers et al., 2003; Nakamura \& Zea, 2010; Peterson et al., 1992; Hirokazu Yoshikawa et al., 2004). Of all the SES indictors reviewed, only income was consistently found to be associated with HIV risk behavior, though the mechanisms of this relationship are unknown. Given that educational attainment is much more developed when sexual attractions, behaviors, and identities begin to take shape, it is reasonable to find little or no association between educational attainment and HIV risk factors. It is also reasonable that associations regarding educational attainment may in fact be a result of racial disparities in educational attainment and not the result of sexuality. Furthermore, the relationship of employment and HIV risk behaviors, influenced by educational attainment, could be complicated by racial disparities in employment among racial minority MSM.

Additional factors, such as partner status and overall workforce participation may explain some of the relationship between employment and HIV risk behaviors. Despite the higher levels of education, the literature shows that MSM overall, and particularly racial minority MSM, earn less money than their heterosexual counterparts further marginalizing this population. These three indicators when examined together demonstrate the realities of diminished SES among racial minority MSM and to a lesser extent how these realities influence HIV risk taking behavior. Furthermore, scholars suggest that additional research be conducted at the encounter level as opposed to the individual level to better contextualize and understand this association. 


\section{SUMMARY}

Experiences of discrimination, whether based on race or sexuality, have been shown to have negative implications for health, including health behaviors (Bennett, Wolin, Robinson, Fowler, \& Edwards, 2005; Gibbons, Gerrard, Cleveland, Wills, \& Brody, 2004; Martin et al., 2003; Yen \& Ragland, 1999). Studies of Asian, Black, and Latino MSM indicate that discrimination may be associated with higher levels of depression and HIV risk behavior (Díaz et al., 2001; Mays \& Cochran, 2001; Meyer, 2003; Stokes \& Peterson, 1998; Yoshikawa et al., 2004). Within this growing body of literature, research has examined the association of discrimination and HIV risk behaviors in which HIV risk is often assessed with measures of recent unprotected anal intercourse and when appropriate taking into account demographic factors, socioeconomic status, partner serostatus, sexual positioning, monogamy, number of sexual partners, and whether or not partners are considered to be main or causal partner.

The research reviewed above indicates that discrimination, most often assessed with a combination of perceived and overt experiences is associated with measures of psychological distress and depression, as measured using the SCL 90-R and the CES-D. However, the research with regard to depression and HIV risk behavior is inconsistent. There are a number of possible reasons those that are depressed may engage in behaviors that put them at risk for HIV.

"Feeling valued and valuable might be necessary preconditions to behaviors that protect one from HIV. People who feel depressed and hopeless might lack the motivation to decrease their risk for HIV. Individuals with low selfesteem may be especially likely to try to hide their same-sex attraction from 
others. For some, showing interest in and learning about HIV and AIDS may be inconsistent with trying to hide their same-sex attractions. Further, people who are psychologically distressed may have difficulty developing long-term intimate relationships, and or they may use causal encounters to help validate their attractiveness and their worth. Casual sex may also serve as a distracter from loneliness or other types of psychological distress" (Stokes \& Peterson, 1998:280).

To better understand this association, research has investigated the influence of alcohol and substance use. When accounting for alcohol use, the association of depression and HIV risk behavior suggests that those who are depressed consume more alcohol when drinking and those that consume higher quantities of alcohol are more likely to engage in UAI. Substance use, specifically polysubstance use, is also associated with depression.

As a means of understanding the resilience and sources of strength among racial minority MSM, the literature highlights a few studies demonstrating that cultural determinants serve as protective factors against depression and HIV risk behavior. The cultural determinants mentioned include social support, racial and sexual identity, and racial and sexual community participation. The literature shows inconsistent findings regarding the impact of some cultural determinants as racial minority MSM are thought to have to negotiate or choose between racial or sexual identities.

The studies have variations in operationalizing key variables, sample populations, and recruitment strategies. Most of these studies are cross-sectional in design limiting the analysis to associations and not allowing for causal inferences to be made regarding the direction of the association. Given these limitations, one consistent theme within the literature is that discrimination differentially impacts 
different racial minority MSM populations. This theme suggests that a one sized fits all approach is inappropriate for HIV prevention among racial minority MSM and acknowledges the need for more research to be conducted with Native MSM as the literature to date only highlights the experiences of Asian, Black, Latino, and Pacific Islander MSM.

Lastly, it is important to note that most large health studies conducted in the United States ignore Natives. Few studies specifically address Native health, and data on Native MSM are even scarcer (Baldwin, Maxwell, Fenaughty, \& Trotter, 2000; Chae \& Walters, 2009). Despite the fact that both public health and social science literature tend to ignore Native MSM overall, there is a growing and yet modest literature pointing out that Native MSM are at greater risk for adverse health outcomes than other Natives (Balsam, Huang, Fieland, Simoni, \& Walters, 2004; Walters, Horwath, \& Simoni, 2001; Walters, 1997). This literature states Native MSM men confront stressors associated with negotiating their multiple oppressed statuses and often must contend with heterosexism in Native communities as well as racism in gay communities (Simoni et al., 2006; Walters et al., 2001; Walters, 1997). To date there have been no previous studies investigating the experiences of discrimination, depression, alcohol and substance use, and HIV risk behaviors among Native MSM. 


\section{CHAPTER 3: THEORY}

The intent of this chapter is to frame HIV infection as biologic expression of social and cultural determinants. The idea is to frame a more complete understanding of how behavior is a result of social and cultural experiences. This chapter will construct a theoretical model that explains how and why discrimination is linked to HIV risk behavior. This chapter starts by locating Native MSM within a historical, cultural, and social context. Meyer's (1995, 2003, 2010) Minority Stress theory is used to explain the association between experiences of discrimination and depression, by explaining how the subordinate social statuses of race and sexuality predict exposure to undue stress from discrimination compared to dominant social statuses. Minority stress is also used in associating depression to maladaptive coping strategies; particularly how depression based on stress related to subordinate statuses leads to alcohol use, substance use, and HIV risk behavior. The Indigenist stress coping theory is used to explain how cultural buffers serve as protective factors against the negative effects of discrimination. This chapter concludes with a discussion of the research questions and how they will inform empirical and theoretical knowledge.

\section{CONTEXTUALIZING TWO- SPIRITS}

Working with indigenous communities requires knowledge and understanding of the relevant historical, cultural, and social context that have influenced these communities, as well as their relationships with the dominant culture, for more than half a millennium. Since first contact, Native people have been 
perceived and represented as a "problem" to be dealt with by way of colonizing projects of domination and subjugation. In the "New World," such forces grew out of religious oppression, forced assimilation, systems of government developed in colonized lands, and implementation of laws that served to further subdue and nearly annihilate Natives and dispossess them of land and other material resources.

Centuries of colonization have had devastating effects on Natives, and specifically two-spirits, that can be assessed today not only in terms of the loss of language, customs, rituals, sacred knowledge, and culture (Duran \& Duran, 1995; Duran, Firehammer, \& Gonzalez, 2008; Gone, 2008; Smith, 1999), but also in the overwhelming disparities in physical and mental health of Natives when compared to the general population. As a result of colonization, Natives also suffer from what is known as historical trauma (Yellow Horse Brave Heart, 2003), also described as a "soul wound" (Duran, Duran, Yellow Horse Brave Heart, \& Yellow Horse-Davis, 1998; Duran et al., 2008). Researchers define these synonymous concepts as "cumulative emotional and psychological wounding, over the lifespan and across generations, emanating from massive group trauma" (Yellow Horse Brave Heart, 2003:7) and the experiences of "some form of historical trauma that continues to cause confusion and suffering in the present" (Duran et al., 2008:288). This inherited legacy of trauma and unresolved grief, through generations of systematic oppression, has engendered residual psychological, physiological, and social harm (Belcourt-Dittloff \& Stewart, 2000). Of the many forms of historical trauma, discrimination is one that continues to traumatize Natives in general and two-spirits specifically. It is with this indigenist (O'Neil, 1986) perspective that places the 
historical and political context of two-spirits at the forefront that I now turn to contemporary explanation of colonizing projects of racialization and heterosexism.

Colonizing projects are best understood as processes of racialization and heterosexism. Racialization is the process by which racial attributes and meanings are projected onto previously nonracial situations (Omi \& Winant, 1986:64). The process of racialization began in the United States with the early contact of Europeans and the concepts of whiteness and purity. Europeans became "white" and the people they encountered became "Indians," thereby creating the social categorization of race. Over time other categories such as "Negros" were created as a process of depicting whites from "others" (Bonilla-Silva, 1997). The social categorization of race and the distinction of whites from others is not the distinct difference in skin coloration, because the difference in skin color is merely a difference. The distinction of whites from others is based on power that happens to fall in line with distinctions in skin color. The racialization process includes this distinction in power as racialization contains the element of hierarchy and social structure. In terms of power, racialization delineates the modes of social inclusion within an encompassing system of categorization. Social inclusion places whites in exclusive positions of power as the structure of racialization consists of a process by which the meaning and valuation of whiteness are derived from the demeaning and devaluation of others (Martinot, 2003:129). The process of "othering" extends beyond race and also applies to gender and sexuality.

Historically, Native societies incorporated gender roles beyond male and female (Tafoya, 1996; Walters, Evans-campbell, Simoni, Ronquillo, \& Bhuyan, 2006). 
Individuals embracing these genders may have dressed; assumed social, spiritual and cultural roles; or engaged in sexual and other behaviors not typically associated with members of their biological sex. From the community's perspective, the fulfillment of social or ceremonial roles and responsibilities was a more important defining feature of gender than sexual behavior or identity. Although there were exceptions, many of the individuals who embodied alternative gender roles or sexual identities were integrated within their community, often occupying highly respected social and ceremonial roles (Walters et al., 2006). Western colonization and Christianization of Native cultures, however, attacked traditional Native conceptions of gender and sexual identity. The colonizing project of heterosexism succeeded in undermining traditional ceremonial and social roles for two-spirits within many tribal communities, replacing traditional acceptance and inclusivity with shaming and condemnation, which are processes that reinforce heterosexism (Tinker, 1993). Heterosexism is the process that assumes all people are heterosexual and stigmatizes, denies, and or denigrates anyone or anything that is non-heterosexual. Since the introduction of Christianity and heterosexism, American society has stigmatized same-sex sexual contact (Simoni et al., 2006).

The concepts of power and hierarchy with respect to racialization, allow for the conceptualization of "racialized social systems," which are best thought of as "societies in which economic, political, social, and ideological levels are partially structured by the placement of actors in racialized categories or races" (BonillaSilva 1997:469). Similarly, concepts of power and hierarchy with respect to heterosexism, allow for conceptualization of "heteronormativity" which is a 
privilege that operates by naturalizing heterosexuality as well as by rendering heterosexuality as the original or the norm, while implicating all other sexualities as deviant or abnormal (Butler, 1993:125-126). As such heterosexism is socially imbedded and institutionalized in the law, military, family, religion, education, and politics. Heterosexism is a resilient system capable of absorbing and appropriating challenges on its edges in order to strengthen itself. Thus, sexual "deviance" from the heterosexual norm can provoke gender and sexual policing that strengthen and further naturalize particular forms of heterosexism.

This is to say that the placement of people into racial and sexual categories also places them into a social hierarchy that produces social relations between races and between sexualities. These social relations produce positions of superiority/inferiority, normality/abnormality, inclusion/exclusion and majority/minority. Those in majority positions within the social hierarchy tend to have access to more resources than those in inferior positions. Moreover, people in majority positions utilize their advantage to resources as a means of creating physical and social boundaries to distinguish themselves from others. These boundaries create racialized and sexualized social relations and practices that comprise the structure of society (Bonilla-Silva, 1997).

Of the social relations and practices, the phenomenon of stigma serves as a catalyst for maintaining the structure of society by separating the majorities from the minorities. Understood as a social marker, stigma identifies those that are socially discredited and discreditable (Goffman, 1963). As such, stigma marks individuals in a marginalizing way allowing for an ideology to explain how 
minorities are inferior to majorities and rationalize the animosity based on differences. Society is socialized to understand and maintain the distinction between majority and minority groups. For example, the use of specific stigmatizing terms in common discourse (i.e. savages, fagots, etc.) serves as a source of continued identification, social distancing, and relational ways of being (Goffman, 1963). The use of these words is one form of discrimination that effectively, perpetually, and often unconsciously limits the life chances of minorities.

Minority groups exist on the lower or outer limits of social desirability and consciousness. Whether racial, sexual, or racial/sexual minorities, these groups are perceived negatively and lesser in society, confining them to lower and outer limits, resulting in their exclusion from the mainstream. The relations and practices associated with maintaining physical and social boundaries are constant, continuing, and cumulative experiences of socially devalued groups (Sue et al., 2007).

Though U.S. history offers many examples of racial and sexual intersections, it is important to consider the multiple identities of two-spirits and their intersections. Over the past twenty years, the concept of "intersectionality" has emerged as an influential approach to understanding the complex facets of discrimination and marginalization in a society whose members can experience marginalization in multiple ways based on their complex identities. The feminist theory of intersectionality encompasses a set of foundational claims and organizing principles for understanding social inequality and its relationship to individuals' marginalized statuses (Choo \& Ferree, 2010; Collins, 1990; Crenshaw, 1991; McCall, 
2005). Intersectional approaches are based on the assumption that individual and group identities are complex and shaped not just by one dimension of identity (race, class, ethnicity, sexuality/sexual orientation, gender, physical disabilities, national origin, disease status, among many others) but rather by the convergence of multiple dimensions of one's identity. As a result, this perspective is useful for providing insights in the simultaneous production of these dimensions of inequality and also permits investigations into understanding how these dimensions are interrelated and how they shape and influence one another (Dill \& Zambrana, 2009; Weber, 2010). With respect to two-spirits, intersectionality challenges one to look at the points of cohesion and fracture within the "racial/sexual minority group", as well as allowing comparisons between racial/sexual minority groups and the dominant group culture. With a theoretical foundation of understanding of racialization and heterosexism, I can now assess explanations of racial and sexual discrimination as a means racial/sexual relations.

\section{THEORETICAL FRAMEWORK: LINKING DISCRIMINATION TO HIV RISK \\ BEHAVIOR}

How we explain the occurrence of HIV infection and its distribution among minority populations depends in part, on conceptual frameworks that guide the collection and interpretation of data, as well as the design and implementation of policies and programs. To date, much of the literature on HIV risk has relied upon theories that explain health disparities in HIV transmission using an individualistic paradigm or theories that explain HIV transmission only at the level of individual 
agency (Cockerham, 2005; Zierler \& Krieger, 1998). However, the HIV behavioral research literature is beginning to document important associations of structural dimensions, such as discrimination, that influence the health behaviors of people (Ayala et al., 2012; Poundstone et al., 2004).

Using minority stress and Indigenist stress coping theories, I explain how discrimination is associated with depression and how depression is associated with HIV risk behaviors. Beginning with the notions that race and sexuality are social constructions that serve as two social determinants of health and these social determinants are "the features of and pathways by which societal conditions affect health" (Krieger, 2001:697). Further, these social determinants explain patterns that enhance HIV/AIDS vulnerability by conceptualizing transmission at a structural level. Conceptualizing HIV transmission at the structural level, allows for an examination of HIV transmission beyond that of the individual level, focusing on social experiences (i.e. discrimination) that affect HIV transmission dynamics and the differential distribution of HIV among racial minority MSM.

Discrimination creates minority stress or a type of harm that is "structured' by historically given processes and forces that conspire- whether through routine, ritual, or, as is more commonly the case, the hard surfaces of life-to constrain agency" (Farmer 2005:40). The constraining of agency is a result of patterns of discrimination based on race/ethnicity, gender, sexual orientation, and other social divisions of difference, that situate minority groups in inferior positions. These inferior positions place members of minority groups at increased risk for depression. As a result of increased levels of depression due to minority stress, 
minorities will partake in stress coping activities and behaviors as a means of minimizing their depression. Maladaptive coping strategies are activities and behaviors that minimize depression while concurrently placing individuals at risk for other health conditions. Examples of maladaptive coping strategies of dealing with minority stress include alcohol use, substance use, and sexual endeavors. Protective factors are types of coping processes that build positive self-esteem and self-concept, thereby reducing minority stress and as such protect against the impacts of discrimination. Both maladaptive coping strategies and protective factors pertain specifically to being two-spirit by capturing the sociohistorical context of being both native and non-heterosexual, making these forms of coping essential to include when working with two-spirit populations.

The conceptual model presented below is an adaption that merges the social epidemiology of HIV model (Poundstone, 2004), minority stress model (Meyer 1995) and the Indigenist Stress Coping model (Walters et al., 2002) depicting how marginalization impacts levels of distress that influence health risk behaviors while accounting for protective factors. 
Figure 1: Conceptual Model

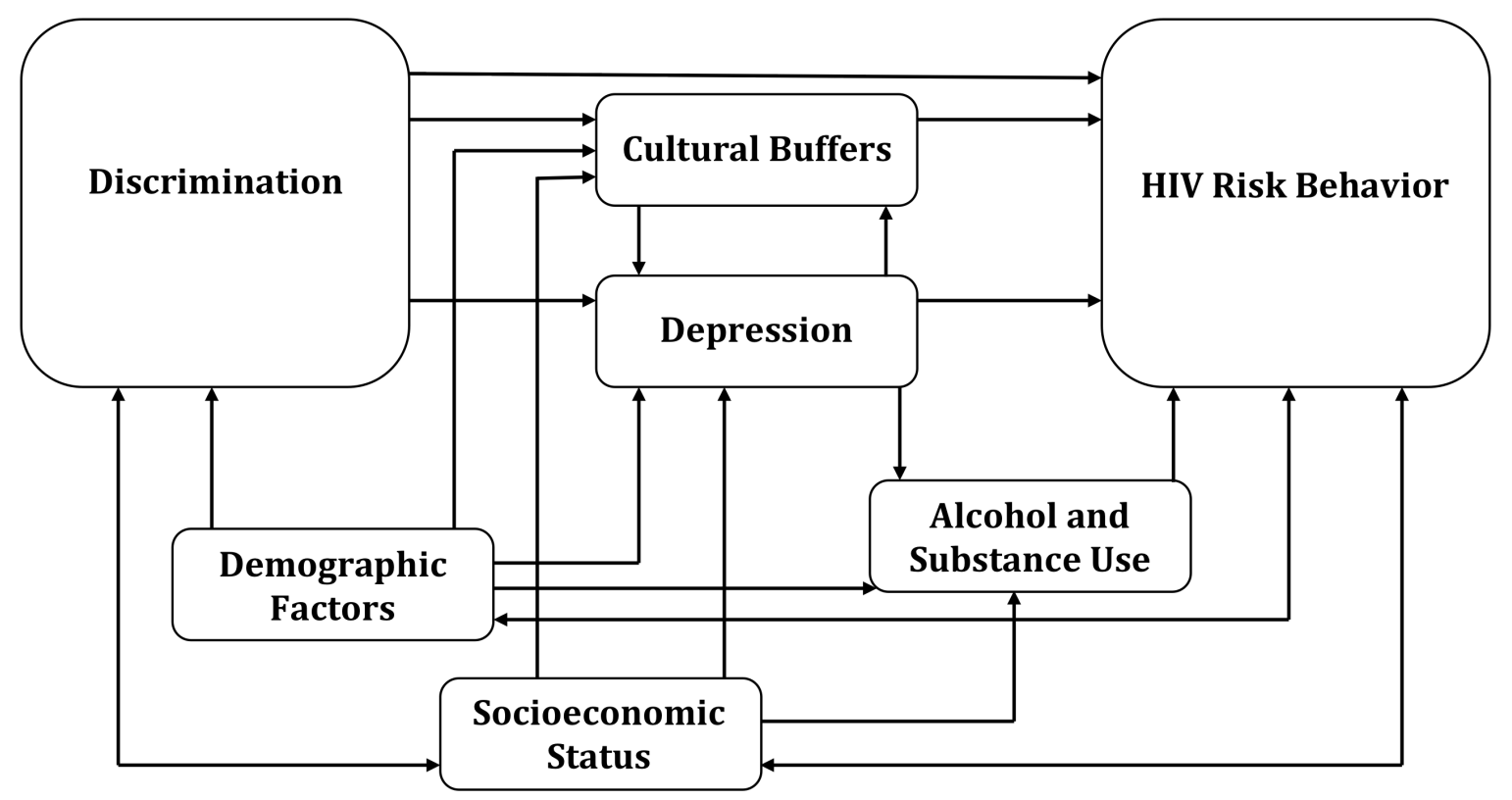

\section{LINKING DISCRIMIANTION TO DEPRESSION}

Prior to the Civil Rights movement discrimination based on race and sexuality were predominantly overt and direct. However, more contemporary forms of discrimination are considered more invisible, subtle, and covert (Dovidio \& Gaertner, 2000; Sue et al., 2007). Dovidio and Gaertner (2000) conceptualize these contemporary forms of discrimination as aversive or "the attitudes of many [nonminorities] who endorse egalitarian values, who regard themselves as nonprejudiced, but who discriminate in subtle, rationalizable ways" (p. 315). The aversive or subtle forms of discrimination have been documented to be responsible for workplace inequities called "micro-inequity" and have been applied to sexual 
minorities as well as racial minorities (Rowe, 1990). One mechanism by which aversive discrimination occurs is microaggressions.

Microaggressions are daily subtle indignations that people experience as a result of their minority status(es). Racial and heterosexist microaggressions serve as mechanisms for aversive racism and heterosexism and often occur below the level of awareness of the aggressor, who is often considered to be well intentioned. Microaggressions focus on the interplay of the aggressor and the recipient, the deconstruction of hidden messages, and the exploration of both internal (psychological health) and external (physical health) consequences (Sue et al., 2007:9). Microaggressions operate with the assumption that each message serving as a microaggression has two components that often contradict each other. The first component is the face value of the message in which a comment, question, or joke appears harmless. The second component is a hidden message, which is often demeaning and detrimental for the recipient. It is the hidden message that serves as the marginalizing mechanism that reaffirms the social hierarchy that locates people within racialized and sexualized categories, and the marginalization of minorities. Listed below are some example microaggressions taken from Sue et al., 2007:

Racial:

A white man checks his wallet as a Black or Latino man approaches or passes them. (Hidden Message: You and your group are criminals.)

An Asian American, born and raised in the United States, is complimented for speaking "good English." (Hidden Message: You are not a true American. You are a perpetual foreigner in your own country.)

Sexual Orientation:

Students use the term "gay" to describe a fellow student who is socially 
ostracized. (Hidden Message: People who are weird, strange, deviant, or different are "gay.")

Two men hold hands in public and are told not to flaunt their sexuality. (Hidden Message: Homosexual displays of affections are abnormal and offensive. Keep it private and to yourselves.)

With an understanding of microaggressions, I turn to minority stress theory to demonstrate how subordinate social statuses of race and sexuality, would predict exposure to undue stress from social discrimination compared to dominant social statuses. Minority stress helps explain the pathway in which discrimination leads toward depression by understanding how stress is associated with minority status. Minority stress theory (Meyer, 1995, 2003, 2010) posits that individuals from marginalized social groups experience excess stress due to their minority status(es), in addition to the general stressors experienced by all people. Moreover, various social structures, institutions, and processes beyond the individual contribute further to the experience of minority stress. Meyer (2003) outlines three assumptions in the conceptualization of minority stress. First, minority stress is unique to the stigmatized group and therefore, is added to general stressors that are experienced by all people. Second, minority stress is chronic given that it is built upon stable underlying social and cultural structures. Finally, minority stress is socially based, that is to say it stems from social processes, institutions, and structures beyond the individual.

Meyer built this theory from the work of Garnets, Herek, and Levy (1990) who suggest that processes of victimization interfere with the perception of the world and lead victims toward self-devaluation and ultimately psychological 
distress. As a result the most explicit sources of minority stress are rejection, discrimination, and violence (Meyer, 2003). The most important aspect of minority stress theory based on discrimination is the context of minority oppression. That is to say that discriminatory acts have a strong impact not because of the event itself but rather because of the cultural meaning that they evoke. For example, a slur directed toward a minority may activate feelings and emotions that are disproportionate to the event itself (Meyer, 1995). Exposure to minority stress, particularly stress resulting from discrimination, might underlie the greater risk for psychological distress (Mays \& Cochran, 2001; Meyer, 1995) and thus depression. Minority stress was originally developed by Brooks (1981) for lesbians. Meyer (1995) expanded it to include gay men and bisexuals (Meyer, 2003) and the work of Nemoto and colleagues $(2003,2006)$ have garnered evidence demonstrating that model is appropriate for transgender individuals as well. Minority stress theory has been used to examine racial discrimination as a source of social stress that is associated with poor mental health outcomes (Chae \& Yoshikawa, 2008; Noh \& Kaspar, 2003). Here I use the minority stress theory to explain how discrimination based on racial and sexuality creates excess stress that is unique to racial and sexual minority groups. Thus racial minority MSM would have the stress of being a sexual minority as well as the added stress of being a racial minority compared to white heterosexual men that would not experience racial and sexual discrimination. As such I argue that the combination of multiple minority statuses and the chronic strains associated with multiple forms of discrimination have particularly devastating effects of the psychological health of 
racial minority MSM.

\section{LINKING DEPRESSION TO MALADAPTIVE COPING BEHAVIORS}

The experience of depression may lead to a variety of coping mechanisms among individuals (Bruce et al., 2008). From a stress and coping perspective, alcohol use, substance use, and sexual endeavors are often conceptualized as maladaptive coping strategies for dealing with depression (Bruce et al., 2008). The explanations for the positive association between depression and maladaptive coping strategies include reducing motivation for self-protective behavior (Baum \& Polsluszny, 1999), stimulating sensation seeking (Kalichman, Tannenbaum, \& Nachimson, 1998), and serving as "cognitive escape" for those experiencing elevated levels of stress (McKirnan, Ostrow, \& Hope, 1996). Regardless of the explanation(s), it is thought that engaging in maladaptive coping strategies is in response to depression created by stigmatization towards one's minority status(es).

The minority stress model helps demonstrate how depression leads to maladaptive coping behaviors. The premise of stress coping theory is that any stress coping strategies that stems from groups with depression are destined to increase maladaptive health behaviors and ultimately increased HIV risk behavior and adverse health outcomes. Though not yet investigated among racial minority MSM, the association between depression and the maladaptive coping strategy of unprotect sex has been documented among adolescent males (Lehrer, Shrier, Gortmaker, \& Buka, 2006; Shrier, Harris, Sternberg, \& Beardsless, 2001), injection drug users (Perdue, Hagan, Thiede, \& Valleroy, 2003), and MSM (Perdue et al., 
2003). Thus it is plausible that engaging in high-risk sexual behavior, as well as alcohol and substance use, may function as maladaptive coping strategies amongst racial minority MSM, including Native MSM in response to stress created by discrimination regarding race and sexuality.

\section{LINKING CULTURAL DETERMINANTS TO DEPRESSION \& MALADATIVE COPING BEHAVIORS}

The notion of cultural determinants stems from the theory of resiliency, which conceptualizes the construct of stress mediators, also known as coping resources (Lazarus \& Folkman, 1984) or cultural buffers in the literature specifically pertaining to Native health (Walters et al., 2002). Stress mediators are resources that individuals can use on their behalf in the presence of stress. The availability of a stress mediator makes an individual more resilient to the adverse effects of stress. On the other hand, the absence of a stress mediator can make an individual more vulnerable to stress (Beresford, 1994). The larger literature of stress coping suggests that stress mediators have two theoretical perspectives, the direct hypothesis and the buffering hypothesis, to explain the association between stress mediators and well being (Lazarus \& Folkman, 1984). The direct hypothesis posits that having stress mediators has a beneficial effect on well being regardless of whether or not individuals are experiencing stress. The buffering hypothesis posits the stress mediators protect individuals from the harmful effects of stress. At least two junctures have been identified where stress mediators can have a buffering 
effect: between stressors and distress, and between stress and health outcome Lazarus \& Folkman, 1984).

The direct hypothesis is criticized for placing too much emphasis on individual agency and is thought to be "irrelevant" to indigenous communities as this line of thinking contains Eurocentric values, methodologies, and conceptualizations of stress and stress processes (Walters et al., 2002). The stress coping theory posited by Walters, Simoni and Evans-Campbell (2002) stems from an Indigenist perspective, conceptualizing Native health within an indigenous framework. Their theory emphasizes more of the buffering hypothesis, suggesting that cultural buffers minimize the relationship between stressors and health outcomes by mitigating the effects of the traumatic stressors, such as ongoing processes of colonization, thereby decreasing high-risk behaviors and strengthening psychological and emotional health (Walters et al., 2002:S106). Walters and colleagues (2002) hypothesize that family and community social support, enculturation, identity attitudes, spiritual coping, and participation in traditional ceremonies and health practices, all serve as cultural buffers.

The Indigenist perspective places cultural buffers in a hierarchy based on an individuals commitment to the group or networks of social relationships (Serpe \& Stryker, 1987; Stryker, 1987). Cultural buffers then can be central to Native's commitment to their racial group as well as their sexual group. As such, cultural buffers may be resources to cope with stress, meaning racial and sexual culture is beneficial to an individual's health by providing a sense of belonging that buffers against the detrimental impact of discrimination (Anderson, 1991). However, it 
should be noted that two-spirits in general, or Native MSM specifically, may not have access to all the above mentioned cultural buffers based on their sexuality, thereby reducing their exposure to protective factors and increasing their risk of adverse health outcomes. The work of Walters, Simoni, and Horwath (2001) highlights that some two-spirits encounter marginalization from other natives based on their sexuality, which diminishes, and in some cases eliminates, the protective effect of social support.

Within the broader LGBT literature, protective factors are thought to also exist (Meyer, 1995; Raymond et al., 2011). These factors include social support, identity attitudes, and acculturation. Similar to those proposed by Walters and colleagues (2002), these LGBT protective factors minimize the relationship between stressors and health outcomes, thereby strengthening psychological and emotional health, decreasing high-risk behaviors, and mitigating the effects of stressors. Specifically, this occurs as information and support are provided within one's social network. This information and support are thought to provide sexual risk reduction strategies, positive feedback and validation for safe sex practices, and an environment that comes well equipped with information and safer sex supplies (Chng \& Geliga-Vargas, 2000; Ratti et al., 2000; Seibt et al., 1995).

For Native MSM, having valued identities disparaged by discriminatory experiences may be especially stressful (Noh et al. 1999 from Mossakowski 2003). The Indigenist stress coping model provides a framework for understanding how Natives cope with stressors stemming from colonization and Christianization (i.e. the processes that have created minority stress). Lastly, the Indigenist stress coping 
model uses a buffer hypothesis to explain how cultural buffers may mediate the impact of discrimination on depression and maladaptive coping behaviors.

\section{DEMOGRAPHIC FACTORS}

Demographic factors that are also known to influence depression, alcohol use, substance use, and HIV risk behaviors among racial minority MSM. It is widely known that rates of substance use and risky sexual behavior vary by age with individuals between 18 and 25 years of age partaking at the highest rates. Epidemiological data also show that young men under the age of 25 that have sex with men are disproportionately infected with HIV (Centers for Disease Control and Prevention, 2009). As stated in the literature review, HIV status and whether or not a person has a partner or significant other influences the level of and frequency of HIV risk behaviors as well as depression and alcohol and substance use. Socioeconomic status indicators are also important to consider when examining these phenomena. Despite the fact that depression, alcohol use, substance use, and risky sexual behaviors appear at all levels of social strata, HIV infection is known to concentrate among the poor, unemployed, and least educated (Farmer, 1999, 2005; Zierler \& Krieger, 1998).

\section{THEORETICAL SUMMARY}

According to the theoretical framework presented above, discrimination (based on race and sexual orientation) is associated with depression, which influences maladaptive coping behaviors (alcohol use, substance use, and HIV risk 
behaviors). It is then further hypothesized that the independent effects of protective factors (such as social support, racial and sexual identity, and racial and sexual community participation) would mitigate the detrimental effects of minority stress by creating positive self-esteem and self-concept thereby diminishing the level of depression and resulting in a reduction of engaging in maladaptive coping behaviors.

\section{RESEARCH QUESTIONS FOR THIS STUDY}

The research questions proposed for the current study take as their starting point the assertion that historically and at present Native MSM are exposed to unique stressors that elevate the risk of psychological distress. Moreover, there is a reason to expect that, for some, the stressors associated with disadvantaged minority statuses create pressures toward maladaptive behavior, including HIV risk behaviors. Informed by the preceding research findings and theoretical framework, the proposed dissertation will seek to address four specific aims by answering the research questions outlined below.

The first aim is designed to determine the existence of a direct relationship between discrimination and HIV risk behavior. I hypothesize that Native MSM will have increased levels of HIV risk behavior associated with racial and heterosexist discrimination. Though there has been research indicating that both forms of discrimination impact HIV risk behavior, no studies have examined these phenomena among Native MSM. 
Specific Aim 1: Examine the extent to which discrimination is associated with HIV risk behaviors.

1. Research Question 1.1: Is racial discrimination associated with HIV risk behavior, and if so, to what extent?

a. Hypothesis 1.1: As racial discrimination increases, the rate of HIV risk behaviors will increase among Native MSM.

2. Research Question 1.2: Is heterosexist discrimination associated with HIV risk behavior, and if so, to what extent?

a. Hypothesis 1.2: As heterosexist discrimination increases, the rate of reported HIV risk behaviors will increase among Native MSM.

The second aim is designed to determine the existence of minority stress among Native MSM. That is to say, assessing the relationship between discrimination and depressive symptoms will establish the level of stress associated with being a racial and sexual minority that goes above and beyond the general stress experienced by the general population. I hypothesize that Native MSM will have increased levels of depressive symptoms associated with racial and heterosexist discrimination.

Specific Aim 2: Examine the extent to which discrimination is associated with depressive symptoms. 
3. Research Question 2.1: Is racial discrimination associated with depressive symptoms, and if so, to what extent?

a. Hypothesis 2.1: As exposures of racial discrimination increase, the rate of reported depressive symptoms will increase among Native MSM.

4. Research Question 2.2: Is heterosexist discrimination associated with depressive symptoms, and if so, to what extent?

a. Hypothesis 2.2: As exposures of heterosexist discrimination increase, the rate of reported depressive symptoms will increase among Native MSM.

The third aim is designed to determine the existence of maladaptive health behaviors as a stress coping mechanism among Native MSM. That is to say, assessing the relationship between depressive symptoms and HIV risk behaviors will establish the stress coping strategies associated with the depressive symptoms as a result of being a racial minority MSM. I hypothesize that Native MSM will have increased levels of HIV risk behaviors associated with depressive symptoms.

Specific Aim 3: Examine the extent to which depressive symptoms are associated with HIV risk behavior.

5. Research Question 3.1: Are depressive symptoms associated with HIV risk behavior, and if so, to what extent? 
a. Hypothesis 3.1: As depressive symptoms increase, the rate of reported HIV risk behaviors will increase among Native MSM.

The fourth aim is designed for the examination of protective factors that minimize the associations between discrimination, depressive symptoms, and HIV risk behavior. I hypothesize that an increase in exposure to protective factors will mediate the associations among discrimination, depressive symptoms, and HIV risk behaviors.

Specific Aim 4: Examine the extent to which the effects of cultural buffers including social support, racial and sexual identity, and racial and sexual community participation mediate the associations of discrimination, depressive symptoms, and HIV risk behaviors.

6. Research Question 4.1: Do social support, racial and sexual identity, and racial and sexual community participation mediate the effect of discrimination on HIV risk behaviors, and if so, to what extent?

a. Hypothesis 4.1.1: As social support increases the association of discrimination on HIV risk behaviors will decrease among Native MSM.

b. Hypothesis 4.1.2: As racial and sexual identity increase the association of discrimination on HIV risk behaviors will decrease among Native MSM. 
c. Hypothesis 4.1.3: As racial and sexual community participation increase the association of discrimination on HIV risk behaviors will decrease among Native MSM.

7. Research Question 4.2: Do social support, racial and sexual identity, and racial and sexual community participation mediate the effect of discrimination on depressive symptoms, and if so, to what extent?

d. Hypothesis 4.2.1: As social support increases the association of discrimination on depressive symptoms will decrease among Native MSM.

e. Hypothesis 4.2.2: As racial and sexual identity increase the association of discrimination on depressive symptoms will decrease among Native MSM.

f. Hypothesis 4.2.3: As racial and sexual community participation increase the association of discrimination on depressive symptoms will decrease among Native MSM.

8. Research Question 4.3: Do social support, racial and sexual identity, and racial and sexual community participation mediate the effect of depressive symptoms on HIV risk behaviors, and if so, to what extent?

a. Hypothesis 4.3.1: As social support increases the association of depressive symptoms and HIV risk behaviors will decrease among Native MSM. 
b. Hypothesis 4.3.1: As racial and sexual identity increases the association of depressive symptoms and HIV risk behaviors will decrease among Native MSM.

c. Hypothesis 4.3.1: As racial and sexual community participation increase the association of depressive symptoms and HIV risk behaviors will decrease among Native MSM.

\section{SUMMARY}

The empirical literature and the theoretical review informed the development of the research questions and hypotheses proposed in this study. Little is known about the influences of these determinants among Native MSM, however it is plausible that Native MSM experience greater exposure to depressive symptoms and maladaptive coping strategies as seen among other racial minority MSM. It is also plausible that cultural buffers serve as protective factors for Native MSM. The proposed study has the potential to make an important contribution to the existing literature, as it will help understand the influence of social and cultural determinants on HIV risk behavior among Native MSM. Additionally, this will add to the compendium of research regarding racial minority MSM and their relationship with HIV transmission. Furthermore, this study can inform and support the notion that HIV prevention needs to begin using a determinants of health perspective that takes into account the relevant historical, cultural, and social context of the populations of interest. 


\section{CHAPTER 4: METHODOLOGY}

\section{The HONOR Project}

The HONOR Project, the first national study of two-spirit individuals (Cassels et al., 2011), was a multisite study funded by the National Institutes of Mental Health to examine the relationships among trauma, coping, and health in exploring Native culture, spirituality, and traditions as a means of decreasing poor health behaviors and outcomes. The Indigenous Wellness Research Institute at the University of Washington conducted this study. The data were collected in collaboration with community-based agencies serving American Indians and Alaska Natives in seven urban sites across the United States: New York City, Los Angeles, San Francisco/Oakland, Tulsa/Oklahoma City, Denver, Minneapolis-St. Paul, and Seattle/Tacoma (Cassels et al., 2011; Chae \& Walters, 2009; Lehavot, Walters, \& Simoni, 2009). Data were collected on discrimination, HIV risk behavior, depressive symptoms, and cultural buffers, making it suitable to explore the research questions.

The HONOR project used semi-structured computer assisted self-interviews to capture the experiences of two-spirits. Participation was open to individuals (a) self-identifying as American Indian, Alaskan Native, or First Nations and either being enrolled in one's tribe or having at least one-quarter in total American Indian blood quantum; (b) self-identifying as gay, lesbian, bisexual, transgender, or twospirit or having had sexual relations with someone of the same sex during the past 12 months; (c) residing, working, or socializing in the study area; (d) being 18 years of age or older; and (e) speaking English (Chae \& Walters, 2009; Lehavot et al., 2009). Given that urban dwelling two-spirits are a small and difficult-to-access 
population, generating a random sample was not ideal to recruit this population. As such the research team utilized a multiple sampling strategy consisting of target sampling within a respondent-driven sampling (RDS) framework to minimized noncoverage, overrepresentation, and other selection biases (Chae \& Walters, 2009; Lehavot et al., 2009).

All participants provided information about their social networks to identify other potential participants. Seattle served as the census site and participation was open to the entire two-spirit community. Individuals were recruited with newsletters, posters, brochures, booths at relevant events, and word-of-mouth. Seattle-participants were given printed coupons and asked to give one to each social network member they identified who met the study criteria. At all of the remaining sites, the study coordinator developed a list of 6 to 8 first wave "seeds" using targeted sampling techniques for the initial wave of RDS. A total of 36 first wave seeds were recruited, of which 33 participated (Lehavot et al., 2009). A second wave of RDS generated 58 "nominees", of which 50 participated (Cassels et al., 2011). Nominees were individuals randomly selected from the participant's network list to be recruited for the study by the participant. There were no significant differences between RDS (seeds and nominees) and volunteer respondents for the cohort overall or by site on key socio-demographic variables (i.e., gender, education, employment, income, or housing)(Cassels et al., 2011; Lehavot et al., 2009). A total of 451 respondents were interviewed between July 2005 and March 2007. Of these, 4 respondents were later excluded due to ineligibility, leaving a total of 447 participants a total response rate of $80.1 \%$ (Cassels et al., 2011). 


\section{PARTICIPANTS}

The analytic sample contains data from a subset of the HONOR Project data. Given that this study is about Native MSM, the analytic sample is comprised of participants that (a) identify as male, (b) have a penis, and (c) have had sex with a man at least once in their lifetime. Participants completed the computer-assisted self-interview containing a battery of demographic, psychosocial, and behavioral measures. The analytic sample contains 221 individuals (49\% of the total HONOR Project sample).

\section{MEASURES}

\section{Demographic Controls}

The HONOR Project collected demographic variables including age (years old at time of interview), HIV status (positive, negative, unknown), and whether participants had a current romantic or sexual partner. Socioeconomic status variables included education (less than high school, high school or GED, some college or vocational training, and college degree or more), household monthly income $(<\$ 1,000, \$ 1,000-\$ 2,000,>\$ 2,000)$, and employment status (working or not working).

\section{Discrimination}

The HONOR project collected information on stress attributable to routine experiences of discrimination using microaggression scales (Cassels et al., 2011; 
Chae \& Walters, 2009; Lehavot et al., 2009). Microaggressions are conceptualized as the everyday verbal, nonverbal, and environmental slights, snubs, or insults, whether intentional or unintentional, that communicate hostile, derogatory, or negative messages to target persons based solely upon their marginalized group membership (Sue et al., 2007). The HONOR project utilized two microaggression scales to assess discrimination. The first is the Microaggressions Distress Scale, developed by Walters (Walters, 1999). This 33-item scale was developed from community-based work with Natives to measure instances of overt and subtle forms of racial discrimination specifically for American Indians and Alaska Natives. Additionally, a Two-Spirit Microaggression Scale, consisting of 32 items, was developed and incorporated to assess discrimination based on sexual orientation. Consistent with stress theories, participants were asked how "distressed" or "bothered" they were over their lifetime with experiences of "unfair treatment by bosses and supervisors because you are Native"; "by being told to 'lighten up' or 'get a sense of humor' over Indian mascots or logos"; "being excluded or asked to leave ceremonies because you are two-spirit"; "seeing stereotyped imagery of two-spirit people such as the Village People." Participants were asked to respond to each item using a Likert scale ranging from 0 (not at all distressed) to 4 (extremely distressed) (Microaggressions Distress Scale $\alpha=0.972$ and Two-Spirit Microaggression Scale $\alpha$ $=0.955)$. Using the 'rowmean' function in STATA, both discrimination scales were operationalized using the mean score, meaning the items from each scale where averaged together to create a composite score known as the mean score. Higher 
mean scores in both variables are associated with increased distress to

discrimination. Both scales can be found in appendix A.

\section{HIV Risk Behavior}

The HONOR project collected information on sexual HIV risk. The sexual risk measures include sexual behaviors over the last 12 months. Respondents indicated whether they had sex with men in the past 12 months, the HIV status of their partners within the past 12 months (positive, negative, or unknown), the number of times they engaged in insertive and receptive anal intercourse, and the frequency of condom use during anal intercourse (never, less than half the time, about half the time, more than half the time, and every single time). Respondents reported their partners' HIV status and were prompted with the following directive:

"The following items ask about your sexual behaviors with partners in the last 12 months who were HIV-positive, HIV-negative, or of unknown HIV status. This refers to your knowledge of their HIV status at the time you had sex with them. For example, if you had sex with someone whose HIV status you did not know but then later you found out to be HIV-positive, this would be considered a partner with HIV-unknown status. Also, we want you to call a sex partner HIV-positive or HIV-negative only if you were quite certain of this information at the time you had sex. For example, they told you their HIV status and you had no reason to doubt them."

From these data, HIV risk behavior was measured with two items, unprotected anal intercourse (UAI) and unprotected receptive anal intercourse (URAI). Both variables are binary indicating whether or not a participant engaged in these activities at least once within the past 12 months. 


\section{Depressive Symptoms}

The HONOR project included the Center for Epidemiologic Studies Short Depression Scale (CES-D 10)(see appendix B). This 10-item scale assesses depression symptoms by asking respondents to recall how they have felt over the past week. Respondents were asked to respond to each item using a Likert scale ranging from 0 (rarely or none of the time) to 3 (all of the time, 5-7 days) ( $\alpha=$ 0.875). This scale has been shown to have high internal reliability with American Indian populations (Manson et al., 1990; Somervell et al., 1993; Curyto et al., 1998; Whitbeck et al., 2002). As consistent with the original design of this scale (Radloff, 1977) the items were combined into one measure of total depressive symptoms, whereby scale items were summed to create a continuous variable with a range of 0 to 30 . Higher scores are associated with more depressive symptoms.

\section{Alcohol and Substance Use}

The HONOR project also collected information on alcohol and substance use. These variables influence sexual behavior and unprotected sex and as such are included as mediating variables. Alcohol use was measured assessing frequency of alcohol consumption over the past 12 months. Participants were asked if they "had any kind of drink containing alcohol within the past 12 months"; "...past 30 days"; and “...past 7 days". These questions were combined into one measure of alcohol use categorizing participants into 4 categories regarding frequency of alcohol use over the past 12 months (not had a drink in the past 12 months; having drank in the 
past 12 month, but not within the past 30 days; drank within the past 30 days, but not in the past 7 days; and drank within the past 7 days).

Substance use was measured assessing the number of different types of drugs participants used within the past 12 months. The HONOR project asked about 9 different types of drugs including any form of methamphetamine, stimulants, 'club' or 'designer' drugs, any form of cocaine, opiates, hallucinogens, inhalants, any form of marijuana, tranquilizers, and erectile dysfunction medication. With the exception of erectile dysfunction medication, participants were asked if they had taken the above substances more than once in the past 12 months to get high, feel better, or change their mood. Erectile dysfunction use was assessed on whether the participant used these medications in the past 12 months without a prescription. These measures were combined into a single variable called substance use. The substance use variable is associated with the number of different types of substances used within the past 12 months.

\section{Social Support}

The HONOR project utilized the Medical Outcomes Study - Social Support Survey (MOS-SS)(see appendix C) originally developed by Sherbourne and Stewart (1991). This 19-item scale assesses the level of participant's perceived social support. Respondents where asked how often someone would have been available to provide various types of support over the past week including "someone to help you with daily chores if you where sick" and "someone to share your private worries and fears with." Respondents were asked to respond to each item using a Likert 
scale ranging from 0 (rarely or none of the time) to 3 (all of the time, 5-7 days) ( $\alpha=$ 0.980). Consistent with the original design of this scale (Sherbourne \& Stewart, 1991) the items were combined into one measure of total perceived social support, whereby items within the scale were summed creating a continuous variable with a range of 0 to 57. Higher scores are associated with more support.

\section{LGBT and Native Identities}

The HONOR project assessed racial and sexual identity using multiple scales as a means of addressing both LGBT and Native perspectives. Both scales can be found in appendix D. With regard to sexual identity attitudes, the HONOR project utilized a scale of LGBT Identity and Acceptance. This scale consists of 31 items (e.g. "I'm glad to be LGBTT-S" and "I'm proud to be part of the LGBT community") in which respondents answered using a Likert scale ranging from 0 (strongly disagree) to 6 (strongly agree) ( $\alpha=0.894$ ). The items from this scale were combined into one measure of LGBT identity, whereby items within the scale were averaged creating a mean score. Higher mean scores are associated with stronger LGBT identity.

Racial identity was assessed using the 17 items from the Actualization subscale from the Urban American Indian Identity Scale (UAIIS). The Actualization subscale assesses the degree to which respondents had a positive integration between self- and group identity with regard to political, ethnic, racial, cultural, and spiritual dimensions of being Native (Chae \& Walters, 2009; Walters, 1995, 1999). The Actualization subscale consists of items such as "I feel at peace with my Indian identity" and "I want to learn more about my cultural heritage." Respondents 
answered each item using a Likert scale ranging from 0 (strongly disagree) to 3 (strongly agree) ( $\alpha=0.885)$. The items from this scale were combined into one measure of Native identity, whereby all items within the scale were averaged creating a mean score. Higher mean scores are associated with stronger Native identity.

\section{LGBT and Native Community Participation}

Community participation, like identity attitudes, assessed LGBT and Native perspectives separately. LGBT community participation was assessed in 3 items (see appendix E). Respondents were asked how often they participated or attended businesses, events, organizations, and groups that specifically cater to the LGBT community in the past twelve months. Respondents were asked to respond to each item using a Likert scale ranging from 0 (never) to 6 (once a day or more) ( $\alpha=$ 0.544). The items from this scale were combined into one measure of LGBT community participation, whereby items within the scale were averaged creating a mean score. Higher mean scores are associated with increased levels of LGBT community participation.

Native community participation was assessed in 8 items (see appendix E). Respondents were asked how often they participated in traditional ceremonies, prayer, and traditional activities (singing and drumming) in the past 12 months. Respondents were asked to respond to each item using a Likert scale ranging from 0 (never) to 5 (once a day or more) ( $\alpha=0.630$ ). The items from this scale were combined into one measure of Native community participation, whereby items 
within the scale were averaged creating a mean score. Higher mean scores are associated with increased levels of Native community participation.

\section{ANALYSIS}

All data preparation and analyses were conducted using STATA (version 12.0). It is important to note that not all of the respondents answered all of the survey questions and as a result, there are some variable with missing values. Of the 18 variables of interest for this study, heterosexist discrimination had 12 missing cases (5.4\%), monthly household income had 3 missing cases (1.4\%), age had 2 missing cases (0.9\%), and alcohol use had 2 missing cases (0.9\%). Additionally, 33 cases had missing values for LGBT identity, LGBT community participation and Native community participation. These 33 cases included the 12 cases missing on heterosexist discrimination, 1 case missing on alcohol use, and one case missing on monthly household income, resulting in a total of 38 (or 17.2\%) cases with data missing in at least one variable.

Statistical testing was conducted to compare the 38 cases with missing data to the 183 cases with no missing data on demographic and behavioral variables (age, HIV status, current partner status, educational attainment, employment status, income and unprotect anal intercourse and unprotected receptive anal intercourse). T-tests were conducted comparing the groups on the continuous variables, and chisquare analyses for the categorical variables. Those with missing data reported significantly less unprotected anal intercourse $\left(\chi^{2}(1)=10.69, \mathrm{p}<.01\right)$, less 
unprotected receptive anal intercourse $\left(\chi^{2}(1)=5.89, \mathrm{p}<.05\right)$, lower levels of education $\left(\chi^{2}(3)=15.99, \mathrm{p}<.01\right)$, lower levels of social support $(\mathrm{t}(219)=-3.21, \mathrm{p}<$ $.01)$, and were significantly more likely to report their HIV serostatus as unknown. Given these differences between groups, those with missing data appear to be a distinct subpopulation within the sample. As such, it was important to retain them as part of the analytic sample as to not bias the findings by excluding this distinctive group of Native MSM.

Multiple imputation was used to generate 5 imputations to address the missing values. This method assumes an arbitrary missing data pattern (Schafer \& Graham, 2002; Schafer, 1997). Multiple imputation has been shown to lead to valid statistical inferences by properly taking into account the uncertainty inherent in missing data (Rubin, 1987) and specifically using the HONOR Project data (Chae \& Walters, 2009). The set of regression analyses, outlined below, were conducted initially among only those with completed data and then again with the imputed data. Overall, the comparison of the analyses between the completed data and the imputed data indicated that the imputed data are likely to produce more conservative estimates, meaning the odds ratios and regression coefficients were smaller among analyses of the imputed data. Despite the difference in the magnitudes of the estimates, the findings were similar between both sets of analyses. Given these similarities among findings and the fact that those with missing data are a distinct subpopulation, the imputed data set was selected for analysis. 


\section{Analysis of Specific Aims and Research Questions}

The goal of this study is to examine the relationship between discrimination and HIV risk behaviors, both directly and indirectly by way of depressive symptoms, among a national sample of Native MSM. Specifically, this study investigates the extent to which racial and heterosexist discrimination are associated with differential levels of UAI and URAI. Furthermore, this study examines whether the relationship between discrimination and HIV risk behavior is mediated by experiences of depressive symptoms. Lastly, this study investigates the effects of social support, racial and sexual identity, and racial and sexual community participation on the associations of interest. A series of regression analyses was conducted to investigate these associations. Listed below are descriptions of the analysis plan, by specific aim.

\section{Specific Aims 1 and 3:}

As a means of addressing research questions 1.1,1.2, and 3.1, a series of logistic regression analyses were conducted. The initial regression modeled the total effects of racial discrimination and heterosexist discrimination on UAI independently and then together. This allowed for an unadjusted estimate of the magnitude of discrimination on UAI. Then the total effect of depressive symptoms on UAI was modeled to determine the unadjusted estimate of the magnitude of depressive symptoms on UAI. Model 5 includes racial discrimination, heterosexist discrimination, and depressive symptoms. Model 6 adjusts for respondents' age, HIV 
status, and whether or not they had a current partner. The following model adjusted for socioeconomic status indicators (education, monthly household income and employment status). The final model adjusts for alcohol and substance use. The same modeling sequence was then performed with URAI (see tables 4 and 5).

\section{Specific Aim 2:}

As a means of addressing research questions 2.1 and 2.2, series of linear regression analyses were conducted. The initial regression modeled the total effect of racial discrimination and heterosexist discrimination on depressive symptoms independently and then together. This allowed for unadjusted estimates of the magnitude of discrimination on depressive symptoms. The next model adjusted for respondents' age, HIV status, and current partner status. Model 5 adjusts for socioeconomic status indicators (education, monthly household income and employment status). The final model adjusted for alcohol and substance use (see Table 6).

\section{Specific Aim 4:}

As a means of addressing research questions 4.1 and 4.3 , a series of logistic regression analyses were conducted. The initial model for this series of regressions is the last model from table 2 that includes racial and heterosexist discrimination, depressive symptoms, age, HIV status, partner status, socioeconomic status indicators, and alcohol and substance use. The subsequent models adjust for social support, racial and sexual identity, and racial and sexual group participation 
independently and then together, models (see models 9 through 12 in table 7). The same modeling sequence was then performed with URAI.

As a means of addressing research questions 4.2, a series of linear regression analyses were conducted. The initial model for this series of regressions is the last model from table 4 that includes racial and heterosexist discrimination, age, HIV status, partner status, socioeconomic status indicators, and alcohol and substance use. The next model adjusted for social support, racial and sexual identity, and racial and sexual group participation independently and then together (see models 7 through 10 in table 9). 


\section{CHAPTER 5: RESULTS DESCRIPTIVE INFORMATION}

Table 3 shows the mean age of the sample was 39.4 years (range: 20-67 years). Thirty-three percent of the individuals reported being HIV-positive. Thirtysix percent reported having a partner at the time of interview. Twenty-eight percent had a high school diploma, while $23 \%$ had a college degree. The majority (54\%) reported they were currently not working. The majority (54\%) reported a monthly household income of less than $\$ 1,000$ (i.e., $<\$ 12,000$ per year). Significance testing was conducted to compare those that had engaged in unprotected anal intercourse (UAI) within the past year and those that had not. Those that engaged in UAI within the past year had significantly higher levels of distress attributed to heterosexist discrimination ( $\mathrm{t}(207)=-2.45, \mathrm{p}<.05)$, higher level of having consumed alcohol within the past week $\left(\chi^{2}(3)=8.51, p<.05\right)$, higher levels of perceived social support $(\mathrm{t}(219)=-2.10, \mathrm{p}<.05)$, and stronger LGBT identity $(\mathrm{t}(219)=-2.49, \mathrm{p}<.05)$ compared to those that had not engaged in UAI within the past year (see Tables 3 \& 4). Similar tests were conducted to compare those that had engaged in URAI within the past year and those that had not. Those that engaged in URAI within the past year were significantly more likely to be HIV-positive $\left(\chi^{2}(2)=10.44, \mathrm{p}<.01\right)$, have a current partner $\left(\chi^{2}(1)=4.29, \mathrm{p}<.05\right)$, have consumed alcohol within the past week ( $\left.\chi^{2}(3)=8.78, p<.05\right)$, and reported higher levels of substance use $(\mathrm{t}(219)=2.90, \mathrm{p}<$ .01), compared to those that had not engaged in URAI within the past year (See Tables 3 \& 4). 
Table 3. Distribution of Demographic Characteristics of Native Men who have Sex with Men, by Unprotected Anal Intercourse and Unprotected Receptive Anal Intercourse: The HONOR Project, 2005-2007

\begin{tabular}{|c|c|c|c|c|c|}
\hline & & \multicolumn{2}{|c|}{ UAI } & \multicolumn{2}{|c|}{ URAI } \\
\hline & Total & No & Yes & No & Yes \\
\hline UAI, no. (\%) & & $\begin{array}{c}134 \\
(60.63)\end{array}$ & $\begin{array}{c}87 \\
(39.37)\end{array}$ & & \\
\hline URAI, no. $(\%)$ & & & & $\begin{array}{c}149 \\
(67.42)\end{array}$ & $\begin{array}{c}72 \\
(32.58)\end{array}$ \\
\hline Racial Discrim. ${ }^{a}$ mean (SD) & $\begin{array}{l}1.40 \\
(1.04)\end{array}$ & $\begin{array}{c}1.39 \\
(1.12)\end{array}$ & $\begin{array}{c}1.42 \\
(0.91)\end{array}$ & $\begin{array}{c}1.42 \\
(1.01)\end{array}$ & $\begin{array}{l}1.36 \\
(0.90)\end{array}$ \\
\hline Heterosexist Discrim. ${ }^{\mathrm{b}}$ mean (SD) & $\begin{array}{c}0.81 \\
(0.84)\end{array}$ & $\begin{array}{c}0.70 \\
(0.79)^{*}\end{array}$ & $\begin{array}{c}0.98 \\
(0.89)^{*}\end{array}$ & $\begin{array}{c}0.76 \\
(0.81)\end{array}$ & $\begin{array}{c}0.92 \\
(0.90)\end{array}$ \\
\hline Age, mean (SD) & $\begin{array}{r}39.42 \\
(10.7)\end{array}$ & $\begin{array}{r}40.27 \\
(11.0)\end{array}$ & $\begin{array}{c}38.14 \\
(10.1)\end{array}$ & $\begin{array}{l}40.38 \\
(10.8)\end{array}$ & $\begin{array}{r}37.47 \\
(10.3)\end{array}$ \\
\hline HIV Status, no. (\%) & & & & & \\
\hline Negative & $\begin{array}{c}134 \\
(60.63)\end{array}$ & $\begin{array}{c}87 \\
(64.93)\end{array}$ & $\begin{array}{c}47 \\
(54.02)\end{array}$ & $\begin{array}{c}100 \\
(67.1)^{* *}\end{array}$ & $\begin{array}{c}34 \\
(47.22)^{* *}\end{array}$ \\
\hline Positive & $\begin{array}{c}72 \\
(32.58)\end{array}$ & $\begin{array}{c}36 \\
(26.87)\end{array}$ & $\begin{array}{c}36 \\
(41.38)\end{array}$ & $\begin{array}{c}38 \\
(25.50)^{* *}\end{array}$ & $\begin{array}{c}34 \\
(47.22)^{* *}\end{array}$ \\
\hline Unknown & $\begin{array}{c}15 \\
(6.79)\end{array}$ & $\begin{array}{c}11 \\
(8.21)\end{array}$ & $\begin{array}{c}4 \\
(4.60)\end{array}$ & $\begin{array}{c}11 \\
(7.38)^{* *}\end{array}$ & $\begin{array}{c}4 \\
(5.56)^{* *}\end{array}$ \\
\hline Has a Current Partner, no. (\%) & & & & & \\
\hline No & $\begin{array}{c}141 \\
(63.80)\end{array}$ & $\begin{array}{c}92 \\
(68.66)\end{array}$ & $\begin{array}{c}49 \\
(56.32)\end{array}$ & $\begin{array}{c}102 \\
(68.46)^{*}\end{array}$ & $\begin{array}{c}39 \\
(54.17)^{*}\end{array}$ \\
\hline 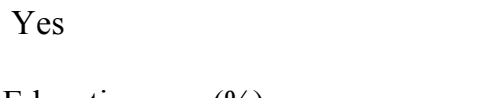 & $\begin{array}{c}80 \\
(36.20)\end{array}$ & $\begin{array}{c}42 \\
(31.34)\end{array}$ & $\begin{array}{c}38 \\
(43.68)\end{array}$ & $\begin{array}{c}47 \\
(31.54)^{*}\end{array}$ & $\begin{array}{c}33 \\
(36.20)^{*}\end{array}$ \\
\hline $\begin{array}{l}\text { Education, no. }(\%) \\
<\text { High School }(<12 \text { yrs })\end{array}$ & 31 & 22 & 9 & 23 & 8 \\
\hline 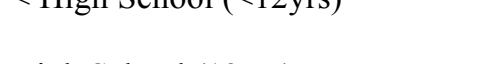 & $(14.03)$ & $(16.42)$ & $(10.34)$ & $(15.44)$ & $(11.11)$ \\
\hline High School (12yrs) & $\begin{array}{c}61 \\
(27.60)\end{array}$ & $\begin{array}{c}35 \\
(26.12)\end{array}$ & $\begin{array}{c}26 \\
(29.89)\end{array}$ & $\begin{array}{c}38 \\
(25.50)\end{array}$ & $\begin{array}{c}23 \\
(31.94)\end{array}$ \\
\hline Some College (13-15yrs) & $\begin{array}{c}79 \\
(35.75)\end{array}$ & $\begin{array}{c}48 \\
(35.82)\end{array}$ & $\begin{array}{c}31 \\
(35.63)\end{array}$ & $\begin{array}{c}52 \\
(34.90)\end{array}$ & $\begin{array}{c}27 \\
(37.50)\end{array}$ \\
\hline College Grad (16+yrs) & $\begin{array}{c}50 \\
(22.62)\end{array}$ & $\begin{array}{c}29 \\
(21.64)\end{array}$ & $\begin{array}{c}21 \\
(24.14)\end{array}$ & $\begin{array}{c}36 \\
(24.16)\end{array}$ & $\begin{array}{c}14 \\
(19.44)\end{array}$ \\
\hline Employment Status, no. (\%) & & & & & \\
\hline Not Working & $\begin{array}{c}120 \\
(54.30)\end{array}$ & $\begin{array}{c}75 \\
(55.97)\end{array}$ & $\begin{array}{c}45 \\
(51.72)\end{array}$ & $\begin{array}{c}81 \\
(54.36)\end{array}$ & $\begin{array}{c}39 \\
(54.17)\end{array}$ \\
\hline Working & $\begin{array}{c}101 \\
(45.70)\end{array}$ & $\begin{array}{c}59 \\
(44.03)\end{array}$ & $\begin{array}{c}42 \\
(48.28)\end{array}$ & $\begin{array}{c}68 \\
(45.64)\end{array}$ & $\begin{array}{c}33 \\
(45.83)\end{array}$ \\
\hline Monthly Household Income \$ (\%) & & & & & \\
\hline$<1000$ & $\begin{array}{c}117 \\
(53.67)\end{array}$ & $\begin{array}{c}66 \\
(50.38)\end{array}$ & $\begin{array}{c}51 \\
(58.62)\end{array}$ & $\begin{array}{c}75 \\
(51.37)\end{array}$ & $\begin{array}{c}42 \\
(58.33)\end{array}$ \\
\hline $1001-2000$ & $\begin{array}{c}48 \\
(22.02)\end{array}$ & $\begin{array}{c}31 \\
(23.66)\end{array}$ & $\begin{array}{c}17 \\
(19.54)\end{array}$ & $\begin{array}{c}31 \\
(21.23)\end{array}$ & $\begin{array}{c}17 \\
(23.61)\end{array}$ \\
\hline $2000+$ & $\begin{array}{c}53 \\
(24.31)\end{array}$ & $\begin{array}{c}34 \\
(25.95)\end{array}$ & $\begin{array}{c}19 \\
(21.84)\end{array}$ & $\begin{array}{c}40 \\
(27.40)\end{array}$ & $\begin{array}{c}13 \\
(18.06)\end{array}$ \\
\hline
\end{tabular}


Table 4. Distribution of Psychosocial and Behavioral Characteristics of Native Men who have Sex with Men, by Unprotected Anal Intercourse and Unprotected Receptive Anal Intercourse: The HONOR Project, 2005-2007

\begin{tabular}{|l|c|cc|cc|}
\hline \multicolumn{2}{|c}{} & \multicolumn{3}{c|}{ UAI } & \multicolumn{2}{c|}{ URAI } \\
\hline & Total & No & Yes & No & Yes \\
\hline Depressive Symptoms mean (SD) & 10.53 & 10.61 & 10.40 & 10.45 & 10.69 \\
& $(6.64)$ & $(7.10)$ & $(5.91)$ & $(6.94)$ & $(6.01)$ \\
Alcohol Use, no. (\%) & & & & & \\
Not within past 12 months & 41 & 28 & 13 & 33 & 8 \\
& $(18.72)$ & $(21.21)^{*}$ & $(14.94)^{*}$ & $(22.45)^{*}$ & $(11.11)^{*}$ \\
Within past 12 months & 27 & 22 & 5 & 22 & 5 \\
& $(12.33)$ & $(16.67)^{*}$ & $(5.75)^{*}$ & $(14.97)^{*}$ & $(6.94)^{*}$ \\
Within past 30 days & 30 & 17 & 13 & 17 & 13 \\
& $(13.70)$ & $(12.88)^{*}$ & $(14.94)^{*}$ & $(11.56)^{*}$ & $(18.06)^{*}$ \\
Within past 7 days & 121 & 65 & 56 & 75 & 46( \\
& $(55.25)$ & $(49.24)^{*}$ & $(55.25)^{*}$ & $(51.02)^{*}$ & $63.89)^{*}$ \\
Substance Use ${ }^{\text {d mean }(S D)}$ & 1.75 & 1.54 & 2.07 & 1.48 & 2.29 \\
& $(1.98)$ & $(1.81)$ & $(2.18)$ & $(1.78)^{* *}$ & $(2.24)^{* *}$ \\
Cultural Buffers, mean (SD) & & & & & \\
Social Support & 48.27 & 45.87 & 51.98 & 45.89 & 53.21 \\
& $(21.3)$ & $(23.8)^{*}$ & $(16.1)^{*}$ & $(23.2)^{*}$ & $(15.5)^{*}$ \\
LGBT Identity & 4.27 & 4.27 & 4.27 & 4.26 & 4.30 \\
& $(0.90)$ & $(0.99)$ & $(0.78)$ & $(0.96)$ & $(0.80)$ \\
Native Identity & 3.46 & 3.45 & 3.48 & 3.46 & 3.46 \\
& $(0.46)$ & $(0.52)$ & $(0.37)$ & $(0.49)$ & $(0.39)$ \\
LGBT Participation & 3.96 & 4.11 & 3.76 & 4.06 & 3.77 \\
& $(1.65)$ & $(1.65)$ & $(1.63)$ & $(1.67)$ & $(1.60)$ \\
Native Participation & 4.28 & 4.51 & 3.98 & 4.42 & 4.02 \\
& $(1.58)$ & $(1.55)^{*}$ & $(1.58)^{*}$ & $(1.58)$ & $(1.56)$ \\
\hline
\end{tabular}




\section{Specific Aim 1: Examine the extent to which discrimination is associated with HIV risk behaviors.}

Research Question 1.1: Is racial discrimination associated with HIV risk behavior, and if so, to what extent?

Research Question 1.2: Is heterosexist discrimination associated with HIV risk behavior, and if so, to what extent?

The results of model 2 shown in table 5, indicate that the unadjusted odds ratio for racial discrimination is not significantly associated with unprotected anal intercourse, (odds ratio[OR]=1.03, standard error $[\mathrm{SE}]=0.14$, ns). After adjusting for heterosexist discrimination the odds ratio depicting UAI based on level of reported racial discrimination becomes less than 1.00 , which would indicate a reduced odds of UAI. However, these results are not significant and could be based purely on chance. The odds ratios for racial discrimination remain less than 1.0 and not significant in models 5 through 8 that adjust for depressive symptoms, age, HIV, partner status, socioeconomic status indicators, and alcohol and substance use. Though racial discrimination might be predicting UAI, it is not statistically significant in this sample as the size of this sample is small and there was very little variation in racial discrimination between those that engaged in UAI in the past 12 months and those that did not.

The results, shown in table 6, indicate that the unadjusted odds ratio for racial discrimination is not significantly associated with unprotected receptive anal intercourse, $(\mathrm{OR}=0.95, \mathrm{SE}=0.13, \mathrm{~ns})$. The unadjusted odds ratio is less than 1.00 and contrary to what was hypothesized. After adjusting for heterosexist discrimination the odds ratio decreases from 0.95 to 0.77 , which again suggests that 
racial discrimination is conditioned by heterosexist discrimination and maybe protective against engaging in UAI. However, these results should be interpreted with caution, as they are not statistically significant. The odds ratios for racial discrimination remain less than 1.0 and not significant in models 5 through 8 that adjust for depressive symptoms, age, HIV, partner status, socioeconomic status indicators, and alcohol and substance use. Given the small sample size and the very little amount of variation between those that reporting engaging in URAI over the past 12 months and those that had not, it is possible that racial discrimination is predicting URAI but the these analyses do not indicate a statistically significant association.

The results of model 1 in table 5 indicate that the unadjusted odds ratio for heterosexist discrimination is associated with unprotected anal intercourse, $(\mathrm{OR}=$ $1.46, \mathrm{SE}=0.24, \mathrm{p}<.05$ ), indicating that distress from heterosexist discrimination is associated with higher odds of engaging in unprotected anal intercourse. Model 3 indicates that distress from heterosexist discrimination remains significantly associated with unprotected anal intercourse even after adjusting for racial discrimination ( $\mathrm{OR}=1.85, \mathrm{SE}=0.45, \mathrm{p} \leq .05)$. In fact, the association is stronger when adjusting for racial discrimination suggesting a suppression effect. The suppression effect in model 3 suggests that the association between heterosexist discrimination and UAI is conditioned by racial discrimination. That is to say that even though racial discrimination is not associated with UAI, adjusting for it in model 3 accounts for some of the variance, which allows for a more true and robust association between heterosexist discrimination and UAI to be shown. The results 
from model 5 in table 2 show that adjusting for depressive symptoms does not impact the association between heterosexist discrimination and $\mathrm{UAI}(\mathrm{OR}=1.85, \mathrm{SE}$ $=0.45, \mathrm{p} \leq .05)$.

Results of model 6 indicate that the association between heterosexist discrimination and UAI becomes more robust (OR $=2.04, \mathrm{SE}=0.52, \mathrm{p} \leq .01)$, suggesting another suppressive effect when adjusting for age, HIV status, and current partner status. That is to say that when adjusting for age, HIV status, and current partner status accounts for some of the variance of the association between heterosexist discrimination and UAI allowing for a more robust relationship to be shown. Model 6 also indicates that HIV status is associated with UAI $(\mathrm{OR}=1.48, \mathrm{SE}=$ $0.24, p \leq .05$ ), meaning those living with HIV have a higher odds of engaging in UAI than those that are HIV negative. Model 7 adjusts for education, employment status, and monthly household income and indicates a suppression effect for heterosexist discrimination $(\mathrm{OR}=2.06, \mathrm{SE}=0.54, \mathrm{p} \leq .01)$, though smaller than what is shown in previous models. Model 7 also indicates a small suppression effect for HIV status $(\mathrm{OR}=1.52, \mathrm{SE}=0.25, \mathrm{p} \leq .05)$ when adjusting for education, employment status, and monthly household income.

The final model in table 5, model 8, adjusts for alcohol and substance use and indicates a suppression effect for heterosexist discrimination $(\mathrm{OR}=2.08, \mathrm{SE}=0.54$, $\mathrm{p} \leq .01$ ). The suppression effect of alcohol and drug use is small in this model but does suggest that alcohol and substance use account for some of the variance of the association between heterosexist discrimination and UAI allowing for a more robust relationship to be shown. Adjusting for alcohol and substance use mediates the 
association between HIV status and UAI (OR $=1.42, \mathrm{SE}=0.24, \mathrm{p} \leq .05)$, suggesting that the addition of alcohol and substance use explains away some of the relationship between HIV status and UAI. Lastly, this model indicates that alcohol use is associated with $\mathrm{UAI}(\mathrm{OR}=1.32, \mathrm{SE}=0.19, \mathrm{p} \leq .05)$. Overall the final model in table 2 suggests that heterosexist discrimination ( $\mathrm{OR}=2.08, \mathrm{SE}=0.54, \mathrm{p} \leq .01)$, HIV status $(\mathrm{OR}=1.42, \mathrm{SE}=0.24, \mathrm{p} \leq .05)$, partner status $(\mathrm{OR}=1.85, \mathrm{SE}=0.58, \mathrm{p} \leq .05)$, and alcohol use (OR $=1.32, \mathrm{SE}=0.19, \mathrm{p} \leq .05)$ are associated with unprotected anal intercourse. This is to say, those reporting higher levels of distress from heterosexist discrimination, HIV-positive serostatus, those with a current partner, and those that drank alcohol within the past 7 days are more likely to engage in unprotected anal intercourse than those reporting lower levels of distress from heterosexist discrimination, HIV-negative serostatus, those without a current partner, and those that have not drank alcohol within the past 12 months.

The results of model 1 in table 6 indicate that the unadjusted odds ratio for heterosexist discrimination is not statistically associated with unprotected receptive anal intercourse, $(\mathrm{OR}=1.23, \mathrm{SE}=0.21, \mathrm{~ns})$. However, model 3 indicates that heterosexist discrimination is approaching significance after adjusting for racial discrimination $(\mathrm{OR}=1.51, \mathrm{SE}=0.36, \mathrm{p} \leq .10)$, suggesting that the association between heterosexist discrimination and URAI is conditioned by racial discrimination. The results from model 5 show that adjusting for depressive symptoms do not impact the association between heterosexist discrimination and URAI (OR $=1.51, \mathrm{SE}=0.36, \mathrm{p} \leq .10)$ as the level of depressive symptoms have no association with URAI $(\mathrm{OR}=1.01, \mathrm{SE}=0.02, \mathrm{~ns})$. 
Model 6 indicates that the association between heterosexist discrimination and URAI is suppressed by age, HIV status, and current partner status $(\mathrm{OR}=1.68, \mathrm{SE}$ $=0.42, \mathrm{p} \leq .05)$. That is to say that adjusting for individual characteristics accounts for some of the variance of the association between heterosexist discrimination and URAI allowing for a more robust relationship to be shown. Model 6 also indicates that age (OR $=0.97, \mathrm{SE}=0.01, \mathrm{p} \leq .05)$, HIV status $(\mathrm{OR}=1.75, \mathrm{SE}=0.30, \mathrm{p} \leq .01)$, and current partner status $(\mathrm{OR}=1.91, \mathrm{SE}=0.60, \mathrm{p} \leq .05)$ are associated with URAI. This means that for every year a person ages they are 3\% less likely to engage in URAI. With respect to HIV status, those living with HIV have a higher odds of engaging in URAI than those that are HIV negative. Lastly, this means that those with a current partner have a 91\% higher odds of engaging in URAI. Model 7 adjusts for socioeconomic status indicators. The results of model 7 indicate a suppression effect for heterosexist discrimination (OR $=1.69, \mathrm{SE}=0.44, \mathrm{p} \leq .05)$, though smaller than what is shown in previous models. Model 7 also indicates a small suppression effect for HIV status (OR $=1.77, \mathrm{SE}=0.31, \mathrm{p} \leq .01)$ and current partner status $(\mathrm{OR}=1.92$, $\mathrm{SE}=0.61, \mathrm{p} \leq .05)$ when adjusting for socioeconomic status indicators. The adjustment for socioeconomic status indicators had no effect on the association between age and URAI as the odds ratio remains unchanged $(\mathrm{OR}=0.97, \mathrm{SE}=0.02, \mathrm{p}$ $\leq .05)$.

The final model in table 6, model 8, adjusts for alcohol and substance use and indicates a small mediating effect for heterosexist discrimination $\mathrm{COR}=1.68, \mathrm{SE}=$ $0.43, \mathrm{p} \leq .05)$. Though small, the mediating effect suggests that alcohol and substance use explains away some of the relationship between heterosexist 
discrimination and URAI. Adjusting for alcohol and substance use mediates the association between HIV status and URAI (OR $=1.42, \mathrm{SE}=0.24, \mathrm{p} \leq .05)$, suggesting that the addition of alcohol and substance use explains away some of the relationship between HIV status and URAI. This model also indicates that the association between current partner status and URAI is suppressed by alcohol and substance use (OR $=2.05, \mathrm{SE}=0.67, \mathrm{p} \leq .05)$. That is to say that adjusting for alcohol and substance use accounts for some of the variance of the association between current partner status and URAI allowing for a more robust relationship to be shown. Lastly, this model indicates that substance use is associated with URAI (OR = $1.18, \mathrm{SE}=0.10, \mathrm{p} \leq .05)$. Overall the final model in table 3 suggests that heterosexist discrimination (OR $=1.68, \mathrm{SE}=0.43, \mathrm{p} \leq .05), \mathrm{HIV}$ status $(\mathrm{OR}=1.67, \mathrm{SE}=0.29, \mathrm{p} \leq$ $.01)$, current partner status (OR $=2.05, \mathrm{SE}=0.67, \mathrm{p} \leq .05)$, and substance use (OR $=$ $1.18, \mathrm{SE}=0.10, \mathrm{p} \leq .05)$ are associated with unprotected receptive anal intercourse. This is to say, those reporting higher levels of heterosexist discrimination, HIVpositive serostatus, those with a current partner, and those that engage in polysubstance use are more likely to engage in unprotected anal intercourse than those reporting lower levels of heterosexist discrimination, HIV-negative serostatus, those without a current partner, and those that do not use substances. 
Table 5. Logistic Regression Models of Native Men who have Sex with Men, Predicting Effects of Heterosexist and Racial Discrimination on Unprotected Anal Intercourse: The HONOR Project, 2005-2007 ( $=221)$

\begin{tabular}{|c|c|c|c|c|c|c|c|c|}
\hline Variable & M1 & M2 & M3 & M4 & M5 & M6 & M7 & M8 \\
\hline $\begin{array}{l}\text { Heterosexist } \\
\text { Discrimination }\end{array}$ & $\begin{array}{l}1.46^{*} \\
(.25)\end{array}$ & & $\begin{array}{l}1.85^{*} \\
(0.45)\end{array}$ & & $\begin{array}{l}1.85^{*} \\
(0.45)\end{array}$ & $\begin{array}{l}2.04 * * \\
(0.52)\end{array}$ & $\begin{array}{l}2.06^{* *} \\
(0.54)\end{array}$ & $\begin{array}{l}2.08^{* *} \\
(0.54)\end{array}$ \\
\hline $\begin{array}{l}\text { Racial } \\
\text { Discrimination }\end{array}$ & & $\begin{array}{c}1.03 \\
(0.14)\end{array}$ & $\begin{array}{c}0.74 \\
(0.14)\end{array}$ & & $\begin{array}{c}0.74 \\
(0.14)\end{array}$ & $\begin{array}{c}0.77 \\
(0.15)\end{array}$ & $\begin{array}{c}0.72 \\
(0.15)\end{array}$ & $\begin{array}{c}0.71 \\
(0.15)\end{array}$ \\
\hline $\begin{array}{l}\text { Depressive } \\
\text { Symptoms }\end{array}$ & & & & $\begin{array}{r}1.00 \\
(0.02)\end{array}$ & $\begin{array}{r}1.00 \\
(0.02)\end{array}$ & $\begin{array}{r}0.99 \\
(0.02)\end{array}$ & $\begin{array}{r}1.00 \\
(0.02)\end{array}$ & $\begin{array}{c}0.98 \\
(0.03)\end{array}$ \\
\hline Age & & & & & & $\begin{array}{c}0.98 \\
(0.01)\end{array}$ & $\begin{array}{c}0.98 \\
(0.01)\end{array}$ & $\begin{array}{r}0.98 \\
(0.02)\end{array}$ \\
\hline HIV Status & & & & & & $\begin{array}{l}1.48^{*} \\
(0.24)\end{array}$ & $\begin{array}{l}1.52 * \\
(0.25)\end{array}$ & $\begin{array}{l}1.42 * \\
(0.24)\end{array}$ \\
\hline $\begin{array}{l}\text { Current Partner } \\
\text { Status }\end{array}$ & & & & & & $\begin{array}{l}1.77^{\dagger} \\
(0.54)\end{array}$ & $\begin{array}{l}1.78^{\dagger} \\
(0.55)\end{array}$ & $\begin{array}{l}1.85^{*} \\
(0.58)\end{array}$ \\
\hline Education & & & & & & & $\begin{array}{c}1.31 \\
(0.24)\end{array}$ & $\begin{array}{c}1.31 \\
(0.25)\end{array}$ \\
\hline $\begin{array}{l}\text { Employment } \\
\text { Status }\end{array}$ & & & & & & & $\begin{array}{r}1.42 \\
(0.49)\end{array}$ & $\begin{array}{c}1.56 \\
(0.55)\end{array}$ \\
\hline $\begin{array}{l}\text { Monthly } \\
\text { Household Income }\end{array}$ & & & & & & & $\begin{array}{r}0.66^{\dagger} \\
(0.15)\end{array}$ & $\begin{array}{r}0.66^{\dagger} \\
(0.15)\end{array}$ \\
\hline Alcohol Use & & & & & & & & $\begin{array}{l}1.32 * \\
(0.19)\end{array}$ \\
\hline Substance Use & & & & & & & & $\begin{array}{r}1.09 \\
(0.09) \\
\end{array}$ \\
\hline Constant & 0.47 & 0.63 & 0.59 & 0.68 & 0.61 & 0.76 & 0.59 & 0.24 \\
\hline $\mathrm{F}$ & $4.97 *$ & 0.04 & $3.45 *$ & 0.05 & $2.36^{\dagger}$ & $2.82 * *$ & $2.25 *$ & $2.24 *$ \\
\hline
\end{tabular}

Note: Logistic regression odds ratios with standard errors in parentheses.

${ }^{\dagger} \mathrm{p} \leq .1 * \mathrm{p} \leq .05 * * \mathrm{p} \leq .01 * * * \mathrm{p} \leq .001$ (Two-Sided Test) 
Table 6. Logistic Regression Models of Native Men who have Sex with Men, Predicting Effects of Heterosexist and Racial Discrimination on Unprotected Receptive Anal Intercourse: The HONOR Project, 2005-2007

\begin{tabular}{|c|c|c|c|c|c|c|c|c|}
\hline Variable & M1 & M2 & M3 & M4 & M5 & M6 & M7 & M8 \\
\hline $\begin{array}{l}\text { Heterosexist } \\
\text { Discrimination }\end{array}$ & $\begin{array}{l}1.23 \\
(.21)\end{array}$ & & $\begin{array}{l}1.51^{\dagger} \\
(0.36)\end{array}$ & & $\begin{array}{l}1.51^{\dagger} \\
(0.36)\end{array}$ & $\begin{array}{l}1.68^{*} \\
(0.42)\end{array}$ & $\begin{array}{l}1.69 * \\
(0.44)\end{array}$ & $\begin{array}{l}1.68^{*} \\
(0.43)\end{array}$ \\
\hline $\begin{array}{l}\text { Racial } \\
\text { Discrimination }\end{array}$ & & $\begin{array}{l}0.95 \\
(0.13) \\
\end{array}$ & $\begin{array}{l}0.77 \\
(0.15)\end{array}$ & & $\begin{array}{l}0.76 \\
(0.15) \\
\end{array}$ & $\begin{array}{l}0.80 \\
(0.16)\end{array}$ & $\begin{array}{l}0.77 \\
(0.17)\end{array}$ & $\begin{array}{l}0.75 \\
(0.16)\end{array}$ \\
\hline $\begin{array}{l}\text { Depressive } \\
\text { Symptoms }\end{array}$ & & & & $\begin{array}{c}1.01 \\
(0.02)\end{array}$ & $\begin{array}{l}1.01 \\
(0.02)\end{array}$ & $\begin{array}{c}1.00 \\
(0.02)\end{array}$ & $\begin{array}{l}1.00 \\
(0.03)\end{array}$ & $\begin{array}{c}0.99 \\
(0.03)\end{array}$ \\
\hline Age & & & & & & $\begin{array}{r}0.97^{*} \\
(0.01)\end{array}$ & $\begin{array}{r}0.97^{*} \\
(0.02)\end{array}$ & $\begin{array}{c}0.98 \\
(0.02)\end{array}$ \\
\hline HIV Status & & & & & & $\begin{array}{r}1.75^{* *} \\
(0.30)\end{array}$ & $\begin{array}{r}1.77 * * \\
(0.31)\end{array}$ & $\begin{array}{r}1.67 * * \\
(0.29)\end{array}$ \\
\hline $\begin{array}{l}\text { Current Partner } \\
\text { Status }\end{array}$ & & & & & & $\begin{array}{l}1.91^{*} \\
(0.60)\end{array}$ & $\begin{array}{l}1.92 * \\
(0.61)\end{array}$ & $\begin{array}{l}2.05^{*} \\
(0.67)\end{array}$ \\
\hline Education & & & & & & & $\begin{array}{c}1.19 \\
(0.23)\end{array}$ & $\begin{array}{c}1.21 \\
(0.24)\end{array}$ \\
\hline $\begin{array}{l}\text { Employment } \\
\text { Status }\end{array}$ & & & & & & & $\begin{array}{c}1.26 \\
(0.45)\end{array}$ & $\begin{array}{c}1.43 \\
(0.53)\end{array}$ \\
\hline $\begin{array}{l}\text { Monthly } \\
\text { Household Income }\end{array}$ & & & & & & & $\begin{array}{c}0.70 \\
(0.17)\end{array}$ & $\begin{array}{c}0.69 \\
(0.17)\end{array}$ \\
\hline Alcohol Use & & & & & & & & $\begin{array}{r}1.31^{\dagger} \\
(0.20)\end{array}$ \\
\hline Substance Use & & & & & & & & $\begin{array}{r}1.18^{*} \\
(0.10)\end{array}$ \\
\hline Constant & 0.40 & 0.52 & 0.50 & 0.46 & 0.46 & 0.70 & 0.62 & 0.22 \\
\hline $\mathrm{F}$ & 1.42 & 0.13 & 1.60 & 0.07 & 1.14 & $3.32 * *$ & $2.41^{* *}$ & $2.44 * *$ \\
\hline
\end{tabular}

Note: Logistic regression odds ratios with standard errors in parentheses.

${ }^{\dagger} \mathrm{p} \leq .1 * \mathrm{p} \leq .05 * * \mathrm{p} \leq .01 * * * \mathrm{p} \leq .001$ (Two-Sided Test) 


\section{Specific Aim 2: Examine the extent to which discrimination is associated with depressive symptoms.}

Research Question 2.1: Is racial discrimination associated with depressive symptoms, and if so, to what extent?

Research Question 2.2: Is heterosexist discrimination associated with depressive symptoms, and if so, to what extent?

The results of model 1 in table 7 indicate that heterosexist discrimination (beta $[\mathrm{b}]=1.14, \mathrm{SE}=0.54, \mathrm{~ns}$ ) is not statistically associated with depressive symptoms. The results of model 2 indicate that racial discrimination $(b=0.49, \mathrm{SE}=$ $0.43, \mathrm{~ns}$ ) is not statistically associated with depressive symptoms. Model 3 includes when adding both heterosexist $(b=-0.37, \mathrm{SE}=0.69, \mathrm{~ns})$ and racial $(b=0.67, \mathrm{SE}=$ $0.55, \mathrm{~ns}$ ) discrimination to the model, neither are statistically associated with depressive symptoms. However model 3 suggests that the associations of heterosexist and racial discrimination are conditioned by each other. Though neither is statistically significant the coefficients for heterosexist discrimination changed from 0.14 to -.37 and changed direction from positive to negative, when adjusting for racial discrimination. The coefficients for racial discrimination change from 0.49 to 0.67 , when adjusting for heterosexist discrimination.

Model 4 indicates that the association between heterosexist discrimination and depressive symptoms is unchanged when adjusting for age, HIV status, and current partner status $(b=-0.37, \mathrm{SE}=0.67, \mathrm{~ns})$. Additionally, the association between racial discrimination and depressive symptoms approaches significance when adjusting for age, HIV status, and current partner status $(b=0.92, S E=0.55, p$ $\leq .10)$. Model 6 indicates that HIV status $(\mathrm{b}=1.34, \mathrm{SE}=0.49, \mathrm{p} \leq .01)$ is associated with increased reporting of depressive symptoms, which means those with HIV- 
positive serostatus reported more instances of depressive symptoms within the past week than those reporting an HIV-negative serostatus. Lastly, model 6 indicates that current partner status $(\mathrm{b}=-2.09, \mathrm{SE}=0.92, \mathrm{p} \leq .05)$ is associated with depressive symptoms, meaning those with a current partner are less likely to report depressive symptoms compared to those that reported not having a current partner. Model 5 indicates that the association between heterosexist discrimination and depressive symptoms is mediated when adjusting for socioeconomic status indicators $(b=-$ $0.23, \mathrm{SE}=0.65, \mathrm{~ns}$ ), though this finding should be interpreted with caution, as the coefficient is not statistically significant. Model 5 indicates that racial discrimination is associated with depressive symptoms after adjusting for socioeconomic status indicators. This result suggests that education, employment status, and monthly household income suppress the relationship between racial discrimination and depressive symptoms. That is to say that adjusting for socioeconomic status indicators accounts for some of the variance of the association between racial discrimination and depressive symptoms allowing for a more robust relationship to be shown. Adjusting for socioeconomic status mediates the associations between HIV status and depressive symptoms $(b=1.05, \mathrm{SE}=0.46, \mathrm{p} \leq .05)$ and current partner status and depressive symptoms $(b=-1.68, \mathrm{SE}=0.86, \mathrm{p} \leq .05)$ suggesting that the addition of socioeconomic status indicators explains away some of the relationship that HIV status and current partner status have with depressive symptoms. Model 6 also indicates that education $(\mathrm{b}=-1.19, \mathrm{SE}=0.51, \mathrm{p} \leq .05)$ and employment status $(\mathrm{b}=-2.18, \mathrm{SE}=0.94, \mathrm{p} \leq .05)$ are associated with depressive symptoms, meaning those with more education and those with current employment 
reported fewer depressive symptoms over the past week. Monthly household income is approaching significance $(\mathrm{b}=-1.04, \mathrm{SE}=0.61, \mathrm{p} \leq .10)$, suggesting that those with higher incomes report fewer depressive symptoms within the past week. The final model, model 6, adjusts for alcohol and substance use and indicates no effect for the relationship between heterosexist discrimination and depressive symptoms $(b=-0.26, \mathrm{SE}=0.64, \mathrm{~ns})$. Model 6 indicates a mediating effect for racial discrimination suggesting that alcohol and substance use explain away some of the relationship between racial discrimination and depressive symptoms. Adjusting for alcohol and substance use also mediates the associations that HIV status $(b=0.47$, $\mathrm{SE}=0.46, \mathrm{~ns})$, current partner status $(\mathrm{b}=-1.56, \mathrm{SE}=0.85, \mathrm{p} \leq .10)$, education $(\mathrm{b}=-$ 1.15, $\mathrm{SE}=0.50, \mathrm{p} \leq .05)$, employment status $(\mathrm{b}=-1.85, \mathrm{SE}=0.93, \mathrm{p} \leq .05)$, and monthly household income $(b=-0.90, \mathrm{SE}=0.60, \mathrm{~ns})$ have with depressive symptoms, suggesting that alcohol and substance use explains away some of the relationship between these associations. This model also indicates an association between alcohol use and depressive symptoms $(b=0.84, \mathrm{SE}=0.36, \mathrm{p} \leq .05)$, meaning that those who reported drinking more often also reported a higher frequency of depressive symptoms over the past week. Additionally, model 6 indicates that substance use is approaching a statistically significant association with depressive symptoms $(b=0.40, \mathrm{SE}=0.22, \mathrm{p} \leq .10)$, meaning that those who engage in polysubstance use were also more likely to report a higher frequency of depressive symptoms in the past week. Overall the final model in table 4 suggests that racial discrimination $(\mathrm{b}=1.13, \mathrm{SE}=0.51, \mathrm{p} \leq .05)$, education $(\mathrm{b}=-1.15, \mathrm{SE}=$ $0.50, \mathrm{p} \leq .05)$, employment $(\mathrm{b}=-1.85, \mathrm{SE}=0.93, \mathrm{p} \leq .05)$, and alcohol use $(\mathrm{b}=0.84$, 
$\mathrm{SE}=0.36, \mathrm{p} \leq .05)$ are associated with depressive symptoms. This is to say, those reporting higher levels of racial discrimination, lower levels of education, not currently employed, and those that drank within the past 7 days are more likely to report a higher frequency of depressive symptoms than those reporting lower levels of racial discrimination, higher levels of education, being currently employed, and those that have not consumed alcohol within the past 12 months. 
Table 7. Linear Regression Models of Native Men who have Sex with Men, Predicting Effects of Heterosexist and Racial Discrimination on Depressive Symptoms: The HONOR Project, 2005-2007

\begin{tabular}{|c|c|c|c|c|c|c|}
\hline Variable & M1 & M2 & M3 & M4 & M5 & M6 \\
\hline $\begin{array}{l}\text { Heterosexist } \\
\text { Discrimination }\end{array}$ & $\begin{array}{l}0.14 \\
(0.54)\end{array}$ & & $\begin{array}{l}-0.37 \\
(0.69)\end{array}$ & $\begin{array}{l}-0.37 \\
(0.67)\end{array}$ & $\begin{array}{l}-0.23 \\
(0.65)\end{array}$ & $\begin{array}{l}-0.26 \\
(0.64)\end{array}$ \\
\hline $\begin{array}{l}\text { Racial } \\
\text { Discrimination }\end{array}$ & & $\begin{array}{l}0.49 \\
(0.43)\end{array}$ & $\begin{array}{l}0.67 \\
(0.55)\end{array}$ & $\begin{array}{l}0.92^{\dagger} \\
(0.55)\end{array}$ & $\begin{array}{l}1.22^{*} \\
(0.52)\end{array}$ & $\begin{array}{l}1.13^{*} \\
(0.51)\end{array}$ \\
\hline Age & & & & $\begin{array}{c}0.01 \\
(0.04)\end{array}$ & $\begin{array}{c}0.04 \\
(0.04)\end{array}$ & $\begin{array}{c}0.06 \\
(0.04)\end{array}$ \\
\hline HIV Status & & & & $\begin{array}{l}1.34 * * \\
(0.49)\end{array}$ & $\begin{array}{l}1.05^{*} \\
(0.46)\end{array}$ & $\begin{array}{c}0.74 \\
(0.46)\end{array}$ \\
\hline $\begin{array}{l}\text { Current Partner } \\
\text { Status }\end{array}$ & & & & $\begin{array}{l}-2.09 * \\
(0.92)\end{array}$ & $\begin{array}{l}-1.68 * \\
(0.86)\end{array}$ & $\begin{array}{l}-1.56^{\dagger} \\
(0.85)\end{array}$ \\
\hline Education & & & & & $\begin{array}{l}-1.19 * \\
(0.51)\end{array}$ & $\begin{array}{l}-1.15^{*} \\
(0.50)\end{array}$ \\
\hline $\begin{array}{l}\text { Employment } \\
\text { Status }\end{array}$ & & & & & $\begin{array}{l}-2.18^{*} \\
(0.94)\end{array}$ & $\begin{array}{l}-1.85^{*} \\
(0.93)\end{array}$ \\
\hline $\begin{array}{l}\text { Monthly } \\
\text { Household Income }\end{array}$ & & & & & $\begin{array}{l}-1.04^{\dagger} \\
(0.61)\end{array}$ & $\begin{array}{l}-0.90 \\
(0.60)\end{array}$ \\
\hline Alcohol Use & & & & & & $\begin{array}{l}0.84^{*} \\
(0.36)\end{array}$ \\
\hline Substance Use & & & & & & $\begin{array}{r}0.40^{\dagger} \\
(0.22) \\
\end{array}$ \\
\hline Constant & 10.41 & 9.84 & 9.88 & 8.75 & 11.01 & 7.60 \\
\hline $\mathrm{F}$ & 0.07 & 1.29 & 0.77 & $2.92 *$ & $6.15 * * *$ & $6.27 * * *$ \\
\hline
\end{tabular}

Note: Linear regression coefficients with standard errors in parentheses.

${ }^{\dagger} \mathrm{p} \leq .1 * \mathrm{p} \leq .05 * * \mathrm{p} \leq .01 * * * \mathrm{p} \leq .001$ (Two-Sided Test) 


\section{Specific Aim 3: Examine the extent to which depressive symptoms are}

associated with HIV risk behavior.

Research Question 3.1: Are depressive symptoms associated with HIV risk behavior, and if so, to what extent?

The results of model 4 shown in table 5 indicate that the unadjusted odds ratio for depressive symptoms is not significantly associated with unprotected anal intercourse, $(\mathrm{OR}=1.00, \mathrm{SE}=0.02, \mathrm{~ns})$. After adjusting for heterosexist and racial discrimination the odds ratio for depressive symptoms remained unchanged (OR = $1.00, \mathrm{SE}=0.02, \mathrm{~ns})$. The odds ratios for depressive symptoms remain not significant in models 6 through 8 that adjust for age, HIV status, partner status, socioeconomic status indicators, and alcohol and substance use.

The results of model 4 in table 6 indicate that the unadjusted odds ratio for depressive symptoms is not significantly associated with unprotected receptive anal intercourse $(\mathrm{OR}=1.01, \mathrm{SE}=0.02, \mathrm{~ns})$. After adjusting for heterosexist and racial discrimination the odds ratio for depressive symptoms remained unchanged $(\mathrm{OR}=$ $1.01, \mathrm{SE}=0.02, \mathrm{~ns})$. The odds ratios for depressive symptoms remain not significant in models 6 through 8 that adjust for age, HIV status, partner status, socioeconomic status indicators, and alcohol and substance use.

\section{Specific Aim 4: Examine the extent to which the effects of cultural determinants including social support, racial and sexual identity, and racial and sexual community participation mediate the associations of discrimination, depressive symptoms, and HIV risk behaviors.}

Research Question 4.1: Do social support, racial and sexual identity, and racial and sexual community participation mediate the effect of discrimination on HIV risk behaviors, and if so, to what extent? 
The first model in table 8 is model 8 from table 5 . The results from model 9 in table 8 indicate that social support is approaching a statistically significant association with unprotected anal intercourse, $(\mathrm{OR}=1.01, \mathrm{SE}=0.01, \mathrm{p} \leq .10)$. However, social support is not a mediator as hypothesized, but rather a predictor of unprotected anal intercourse. This result means that higher levels of social support are associated with higher odds of UAI. When adjusting for social support, the association between heterosexist discrimination and UAI becomes more robust suggesting the social support has a suppression effect on this relationship (OR = $2.19, \mathrm{SE}=0.60, \mathrm{p} \leq .01)$. That is to say that adjusting for social support accounts for some of the variance that allows for a more robust association between heterosexist discrimination and UAI to be shown. Model 9 also indicates the association between racial discrimination and $\mathrm{UAI}$ is approaching significance $(\mathrm{OR}=0.69, \mathrm{SE}=0.15, \mathrm{p} \leq$ $.10)$, which also suggests that social support mediates this association. However, racial discrimination is approaching significance and this finding should be interpreted with caution. Social support mediates the association between HIV status and UAI (OR $=1.41, \mathrm{SE}=0.25, \mathrm{p} \leq .05)$ as well as the association between current partner status and $\mathrm{UAI}(\mathrm{OR}=1.67, \mathrm{SE}=0.54, \mathrm{~ns})$, meaning that adjusting for social support explains away a small proportion of the relationship between HIV status and UAI and explains away the relationship between current partner status and UAI. This model indicates that monthly household income is negatively associated with UAI (OR $=0.63, \mathrm{SE}=0.15, \mathrm{p} \leq .05)$, meaning that for every higher level of income one obtains, the odds of engaging in UAI decreases by $37 \%$. Additionally, social support mediates the association between monthly household 
income and UAI, meaning that adjusting for social support explains away some of the relationship between monthly household income and UAI.

The results from model 10 in table 8 indicate that neither LGBT identity (OR $=1.01, \mathrm{SE}=0.21, \mathrm{~ns})$ nor Native identity $(\mathrm{OR}=1.24, \mathrm{SE}=0.44, \mathrm{~ns})$ are associated with UAI. However, both LGBT and Native identity appear to be predictors of unprotected anal intercourse as opposed to mediators as hypothesized. These findings should be interpreted with caution, as neither association is statistically significant. Compared to model 8, model 10 adjusts for LGBT and Native identity and indicates that the association between heterosexist discrimination and UAI becomes more robust suggesting the LGBT and Native identity have a suppression effect on this relationship ( $\mathrm{OR}=2.12, \mathrm{SE}=0.57, \mathrm{p} \leq .01)$. That is to say that adjusting for LGBT and Native identity accounts for some of the variance that allows for a more true and robust association between heterosexist discrimination and UAI to be shown. Model 10 also indicates a small suppression effect for the association between HIV status and UAI (OR $=1.43, \mathrm{SE}=0.24, \mathrm{p} \leq .05)$. LGBT and Native identity do not impact the associations of current partner status $(\mathrm{OR}=1.85, \mathrm{SE}=$ $0.58, \mathrm{p} \leq .05)$, monthly household income (OR $=0.66, \mathrm{SE}=0.15, \mathrm{p} \leq .10)$, and alcohol use $(\mathrm{OR}=1.32, \mathrm{SE}=0.19, \mathrm{p} \leq .05)$ with $\mathrm{UAI}$ as the odds ration remain unchanged.

The results from model 11 in table 8 indicate that LGBT participation $(\mathrm{OR}=$ $0.82, \mathrm{SE}=0.08, \mathrm{p} \leq .10)$ is approaching significance in its association with UAI. Native participation is not associated with UAI (OR $=0.96, \mathrm{SE}=0.11, \mathrm{~ns})$. Compared to model 8, model 11 adjusts for LGBT and Native participation, the association 
between heterosexist discrimination is mediated ( $\mathrm{OR}=1.90, \mathrm{SE}=0.52, \mathrm{p} \leq .05)$ when adjusting for LGBT and Native participation. Additionally model 11 indicates the association between racial discrimination and UAI is approaching significance $(\mathrm{OR}=0.68, \mathrm{SE}=0.15, \mathrm{p} \leq .10)$, which also suggests that LGBT and Native identity mediate this association. However, racial discrimination is approaching significance and this finding should be interpreted with caution. Model 11, compared to model 8, indicates a small suppression effect for the association between HIV status and UAI $(\mathrm{OR}=1.45, \mathrm{SE}=0.26, \mathrm{p} \leq .05)$, a moderate suppression effect for the association between alcohol use and UAI (OR $=1.37, \mathrm{SE}=0.20, \mathrm{p} \leq .05)$, and a larger suppression effect for the association between current partner status and UAI (OR = $2.18, \mathrm{SE}=0.73, \mathrm{p} \leq .05$ ). This model indicates that monthly household income is negatively associated with UAI (OR $=0.63, \mathrm{SE}=0.15, \mathrm{p} \leq .05)$, meaning higher levels of income are associated with lower odds of engaging in UAI. Additionally, LGBT and Native participation mediate this association.

Model 12 in table 8 adjusts for all 5 cultural buffers, the results indicate that LGBT participation $(\mathrm{OR}=0.83, \mathrm{SE}=0.09, \mathrm{p} \leq .10)$ is approaching significance, suggesting that higher levels of participation within the LGBT community could be associated with lower odds of UAI. All other cultural buffers were not statistically significant in this model. Compared to model 8, the association between heterosexist discrimination and UAI, in model 12, becomes more robust suggesting that the collective of cultural buffers has a small suppression effect on this relationship $(\mathrm{OR}=2.09, \mathrm{SE}=0.61, \mathrm{p} \leq .05)$. That is to say that adjusting for cultural buffer accounts for some of the variance that allows for a more true and robust 
association between heterosexist discrimination and UAI to be shown. Additionally model 12 indicates the association between racial discrimination and UAI is approaching significance $(\mathrm{OR}=0.64, \mathrm{SE}=0.15, \mathrm{p} \leq .10)$, which also suggests that LGBT and Native identity mediate this association. However, racial discrimination is approaching significance and this finding should be interpreted with caution. Model 12, compared to model 8, indicates a small suppression effect for the association between HIV status and UAI (OR $=1.43, \mathrm{SE}=0.26, \mathrm{p} \leq .05)$, a small suppression effect for alcohol use $(\mathrm{OR}=1.35, \mathrm{SE}=0.20, \mathrm{p} \leq .05)$, and a moderate suppression effect for current partner status ( $\mathrm{OR}=1.98, \mathrm{SE}=0.67, \mathrm{p} \leq .05)$. This model indicates that monthly household income is associated with UAI $(\mathrm{OR}=0.60, \mathrm{SE}=0.15, \mathrm{p} \leq$ .05), meaning higher levels of income are associated with lower odds of engaging in UAI. This association is mediated when adjusting for all cultural buffers. Overall, when adjusting for all of the cultural buffers, those most likely to engage in UAI are those reporting higher levels of heterosexist discrimination, HIV-positive serostatus, having a current partner, earning less than $\$ 1000.00$ per month, having drank in that past week, and those not engaging with the LGBT community. 
Table 8. Logistic Regression Models of Native Men who have Sex with Men, Mediating Effects of Cultural Buffers on the Association between Heterosexist and Racial Discrimination on Unprotected Anal Intercourse: The HONOR Project, 2005$2007(\mathrm{~N}=221)$

\begin{tabular}{|c|c|c|c|c|c|}
\hline Variable & M8 & M9 & M10 & M11 & M12 \\
\hline $\begin{array}{l}\text { Heterosexist } \\
\text { Discrimination }\end{array}$ & $\begin{array}{l}2.08 * * \\
(0.54)\end{array}$ & $\begin{array}{l}2.19 * * \\
(0.60)\end{array}$ & $\begin{array}{l}2.12 * * \\
(0.57)\end{array}$ & $\begin{array}{l}1.90^{*} \\
(0.52)\end{array}$ & $\begin{array}{l}2.09 * \\
(0.61)\end{array}$ \\
\hline $\begin{array}{l}\text { Racial } \\
\text { Discrimination }\end{array}$ & $\begin{array}{c}0.71 \\
(0.15)\end{array}$ & $\begin{array}{l}0.69^{\dagger} \\
(0.15)\end{array}$ & $\begin{array}{l}0.69 \\
(0.15)\end{array}$ & $\begin{array}{l}0.68^{\dagger} \\
(0.15)\end{array}$ & $\begin{array}{l}0.64^{\dagger} \\
(0.15)\end{array}$ \\
\hline $\begin{array}{l}\text { Depressive } \\
\text { Symptoms }\end{array}$ & $\begin{array}{c}0.98 \\
(0.03)\end{array}$ & $\begin{array}{l}1.00 \\
(0.03)\end{array}$ & $\begin{array}{c}0.99 \\
(0.03)\end{array}$ & $\begin{array}{c}0.99 \\
(0.03)\end{array}$ & $\begin{array}{c}1.01 \\
(0.03)\end{array}$ \\
\hline Age & $\begin{array}{c}0.98 \\
(0.02)\end{array}$ & $\begin{array}{c}0.99 \\
(0.01)\end{array}$ & $\begin{array}{c}0.98 \\
(0.01)\end{array}$ & $\begin{array}{c}0.99 \\
(0.02)\end{array}$ & $\begin{array}{c}0.99 \\
(0.02)\end{array}$ \\
\hline HIV Status & $\begin{array}{l}1.42 * \\
(0.24)\end{array}$ & $\begin{array}{l}1.41^{*} \\
(0.25)\end{array}$ & $\begin{array}{l}1.43^{*} \\
(0.24)\end{array}$ & $\begin{array}{l}1.45^{*} \\
(0.26)\end{array}$ & $\begin{array}{l}1.43^{*} \\
(0.26)\end{array}$ \\
\hline Current Partner & $\begin{array}{l}1.85^{*} \\
(0.58)\end{array}$ & $\begin{array}{l}1.67 \\
(0.54)\end{array}$ & $\begin{array}{l}1.85^{*} \\
(0.58)\end{array}$ & $\begin{array}{l}2.18^{*} \\
(0.73)\end{array}$ & $\begin{array}{l}1.98^{*} \\
(0.67)\end{array}$ \\
\hline Education & $\begin{array}{c}1.31 \\
(0.25)\end{array}$ & $\begin{array}{c}1.24 \\
(0.24)\end{array}$ & $\begin{array}{c}1.30 \\
(0.25)\end{array}$ & $\begin{array}{c}1.29 \\
(0.26)\end{array}$ & $\begin{array}{c}1.22 \\
(0.25)\end{array}$ \\
\hline $\begin{array}{l}\text { Currently } \\
\text { Employed }\end{array}$ & $\begin{array}{c}1.56 \\
(0.55)\end{array}$ & $\begin{array}{c}1.63 \\
(0.58)\end{array}$ & $\begin{array}{c}1.58 \\
(0.56)\end{array}$ & $\begin{array}{c}1.55 \\
(0.56)\end{array}$ & $\begin{array}{c}1.67 \\
(0.62)\end{array}$ \\
\hline Current Income & $\begin{array}{c}0.66^{\dagger} \\
(0.15)\end{array}$ & $\begin{array}{l}0.63^{*} \\
(0.15)\end{array}$ & $\begin{array}{c}0.66^{\dagger} \\
(0.15)\end{array}$ & $\begin{array}{l}0.63^{*} \\
(0.15)\end{array}$ & $\begin{array}{l}0.60^{*} \\
(0.15)\end{array}$ \\
\hline Alcohol Use & $\begin{array}{l}1.32 * \\
(0.19)\end{array}$ & $\begin{array}{r}1.32^{\dagger} \\
(0.19)\end{array}$ & $\begin{array}{l}1.32 * \\
(0.19)\end{array}$ & $\begin{array}{l}1.37^{*} \\
(0.20)\end{array}$ & $\begin{array}{l}1.35^{*} \\
(0.20)\end{array}$ \\
\hline Substance Use & $\begin{array}{r}1.09 \\
(0.09)\end{array}$ & $\begin{array}{r}1.09 \\
(0.09)\end{array}$ & $\begin{array}{c}1.26 \\
(0.25)\end{array}$ & $\begin{array}{c}1.12 \\
(0.10) \\
\end{array}$ & $\begin{array}{c}1.12 \\
(0.10) \\
\end{array}$ \\
\hline Social Support & & $\begin{array}{r}1.01^{\dagger} \\
(0.01)\end{array}$ & & & $\begin{array}{c}1.01 \\
(0.01)\end{array}$ \\
\hline LGBT Identity & & & $\begin{array}{c}1.01 \\
(0.21)\end{array}$ & & $\begin{array}{c}0.90 \\
(0.20)\end{array}$ \\
\hline $\begin{array}{l}\text { Native Identity } \\
\text { (Actualization) }\end{array}$ & & & $\begin{array}{c}1.24 \\
(0.44)\end{array}$ & & $\begin{array}{c}1.12 \\
(0.43)\end{array}$ \\
\hline $\begin{array}{l}\text { LGBT } \\
\text { Participation }\end{array}$ & & & & $\begin{array}{c}0.82^{\dagger} \\
(0.08)\end{array}$ & $\begin{array}{c}0.83 \\
(0.09)\end{array}$ \\
\hline $\begin{array}{l}\text { Native } \\
\text { Participation }\end{array}$ & & & & $\begin{array}{c}0.96 \\
(0.11)\end{array}$ & $\begin{array}{c}0.96 \\
(0.12)\end{array}$ \\
\hline Constant & 0.24 & 0.11 & 0.11 & 0.76 & 0.35 \\
\hline $\mathrm{F}$ & $2.24 *$ & $2.20 * *$ & $1.87 *$ & $2.04^{* *}$ & $1.72 *$ \\
\hline
\end{tabular}

Note: Logistic regression odds ratios with standard errors in parentheses.

${ }^{\dagger} \mathrm{p} \leq .1 * \mathrm{p} \leq .05 * * \mathrm{p} \leq .01 * * * \mathrm{p} \leq .001$ (Two-Sided Test) 
The results from model 9 in table 9 indicate that social support is associated with unprotected receptive anal intercourse, $(\mathrm{OR}=1.02, \mathrm{SE}=0.01, \mathrm{p} \leq .05)$. However, social support is not a mediator as hypothesized, but rather a predictor of unprotected receptive anal intercourse. This result means that higher levels of social support are associated with higher odds of engaging in unprotected receptive anal intercourse. When adjusting for social support, the association between heterosexist discrimination and URAI becomes more robust suggesting the social support has a suppression effect on this relationship $(\mathrm{OR}=1.81, \mathrm{SE}=0.49, \mathrm{p} \leq .05)$. That is to say that adjusting for social support accounts for some of the variance that allows for a more true and robust association between heterosexist discrimination and URAI to be shown. Model 9 also indicates the association between racial discrimination and URAI is slightly mediated. However, the association is not significant and this finding should be interpreted with caution. Social support has a mediating effect on the association between HIV status and URAI (OR $=1.65, \mathrm{SE}=0.29, \mathrm{p} \leq .01)$ as well as the association between current partner status and URAI $(\mathrm{OR}=1.83, \mathrm{SE}=0.61, \mathrm{p} \leq$ .10), meaning that adjusting for social support explains away a small proportion of the relationship between HIV status and URAI and explains away the relationship between current partner status and URAI. This model indicates that monthly household income $(\mathrm{OR}=0.65, \mathrm{SE}=0.16, \mathrm{p} \leq .10)$ and alcohol use $(\mathrm{OR}=1.30, \mathrm{SE}=$ $0.20, \mathrm{p} \leq .10$ ) are approaching a statistically significant association with URAI. Furthermore, adjusting for social support mediates the associations between monthly household income and alcohol use with URAI, meaning that adjusting for social support explains away some of the relationship between these variables and 
UAI. Lastly, substance use is associated with URAI (OR $=1.17, \mathrm{SE}=0.10, \mathrm{p} \leq .05)$, meaning that for each additional substance used, the odds of engaging in URAI increases by $17 \%$.

The results from model 10 in table 9 indicate that neither LGBT identity (OR $=1.09, \mathrm{SE}=0.24, \mathrm{~ns})$ nor Native identity $(\mathrm{OR}=1.05, \mathrm{SE}=0.38, \mathrm{~ns})$ are associated with URAI. LGBT identity appears to be predicting unprotected receptive anal intercourse, whereas Native identity appears to have a protective effect against URAI. However, these findings should be interpreted with caution, as neither association is statistically significant. Compared to model 8, model 10 adjusts for LGBT and Native identity and indicates that these variables have no effect on the association between heterosexist discrimination and URAI (OR $=1.68, \mathrm{SE}=0.44, \mathrm{p} \leq$ .05). Model 10 also indicates that adjusting for LGBT and Native identity does not effect the associations of URAI with HIV status (OR $=1.67, \mathrm{SE}=0.30, \mathrm{p} \leq .01)$ and current partner status $(\mathrm{OR}=1.67, \mathrm{SE}=0.30, \mathrm{p} \leq .05)$, and the odds ratios remain unchanged between models 8 and 10. This model also suggests a small suppression effect for alcohol use $(\mathrm{OR}=1.17, \mathrm{SE}=0.10, \mathrm{p} \leq .10)$, though this should be interpreted with caution as this association is approaching statistical significance. Lastly, substance use is associated with URAI in this model suggesting that for every additional substance used in the past 12 months, the odds of engaging in URAI increases by $17 \%$.

The results from model 11 in table 9 indicate that LGBT participation is significantly associated with URAI (OR $=0.80, \mathrm{SE}=0.09, \mathrm{p} \leq .05)$, whereas Native participation (OR $=0.99, \mathrm{SE}=0.12, \mathrm{~ns})$ is not associated with URAI. Compared to 
model 8, model 11 adjusts for LGBT and Native participation, the association between heterosexist discrimination is mediated $(\mathrm{OR}=1.51, \mathrm{SE}=0.40, \mathrm{~ns})$ when adjusting for LGBT and Native participation. Model 11, compared to model 8, indicates a small suppression effect for the association between HIV status and URAI $(\mathrm{OR}=1.73, \mathrm{SE}=0.32, \mathrm{p} \leq .01)$, a moderate suppression effect for the association between current partner status and URAI (OR $=2.34, \mathrm{SE}=0.81, \mathrm{p} \leq .05)$, and a small suppression effect for the association between substance use and URAI (OR $=1.20$, $\mathrm{SE}=0.11, \mathrm{p} \leq .05$ ). Though only approaching statistical significance, the association between alcohol use and URAI is slightly suppressed when adjusting for LGBT and Native participation.

Model 12 in table 9 adjusts for all 5 cultural buffers, the results indicate that social support $(\mathrm{OR}=1.02, \mathrm{SE}=0.01, \mathrm{p} \leq .05)$ is associated with URAI, meaning higher levels of social support are associated with higher odds of engaging in URAI. Additionally, LGBT participation $(\mathrm{OR}=0.82, \mathrm{SE}=0.09, \mathrm{p} \leq .10)$ is approaching significance, suggesting that higher levels of participation within the LGBT community could be associated with lower odds of URAI. All other cultural buffers were not statistically significant in this model. Compared to model 8 , the association between heterosexist discrimination and URAI, in model 12, is explained away suggesting that the collective of cultural buffers has a mediating effect on this relationship $(\mathrm{OR}=1.64, \mathrm{SE}=0.47, \mathrm{p} \leq .10)$. Additionally comparing model 8 to model 12, the association between racial discrimination and URAI remains not significant though the collective of cultural buffers does have a slight mediating effect $(\mathrm{OR}=0.72, \mathrm{SE}=0.18, \mathrm{~ns})$, though this findings should be interpreted with 
caution. Model 12 also indicates a small suppression effects for the associations between HIV status and URAI (OR $=1.71, \mathrm{SE}=0.32, \mathrm{p} \leq .01)$, current partner status and URAI (OR $=2.08, \mathrm{SE}=0.74, \mathrm{p} \leq .05)$, and substance use and URAI (OR $=1.20, \mathrm{SE}$ $=0.11, \mathrm{p} \leq .05)$. This model indicates that alcohol is approaching a statistically significant association with URAI $(\mathrm{OR}=1.32, \mathrm{SE}=0.21, \mathrm{p} \leq .10)$. This association is mediated when adjusting for all cultural buffers. Overall, when adjusting for all of the cultural buffers, the association between heterosexist discrimination and URAI is explained away and those most likely to engage in URAI are those reporting HIVpositive serostatus, having a current partner, those who engage in polysubstance use, and those with higher levels of reported social support. 
Table 9. Logistic Regression Models of Native Men who have Sex with Men, Mediating Effects of Cultural Buffers on the Association between Heterosexist and Racial Discrimination on Unprotected Receptive Anal Intercourse: The HONOR Project, 2005-2007 (N=221)

\begin{tabular}{|c|c|c|c|c|c|}
\hline Variable & M8 & M9 & M10 & M11 & M12 \\
\hline $\begin{array}{l}\text { Heterosexist } \\
\text { Discrimination }\end{array}$ & $\begin{array}{l}1.68^{*} \\
(0.43) \\
\end{array}$ & $\begin{array}{l}1.81^{*} \\
(0.49) \\
\end{array}$ & $\begin{array}{l}1.68^{*} \\
(0.44) \\
\end{array}$ & $\begin{array}{l}1.51 \\
(0.40)\end{array}$ & $\begin{array}{l}1.64^{\dagger} \\
(0.47)\end{array}$ \\
\hline $\begin{array}{l}\text { Racial } \\
\text { Discrimination }\end{array}$ & $\begin{array}{l}0.75 \\
(0.16)\end{array}$ & $\begin{array}{l}0.72 \\
(0.16)\end{array}$ & $\begin{array}{l}0.76 \\
(0.17)\end{array}$ & $\begin{array}{l}0.75 \\
(0.17)\end{array}$ & $\begin{array}{l}0.72 \\
(0.18)\end{array}$ \\
\hline $\begin{array}{l}\text { Depressive } \\
\text { Symptoms }\end{array}$ & $\begin{array}{c}0.99 \\
(0.03)\end{array}$ & $\begin{array}{c}1.01 \\
(0.03)\end{array}$ & $\begin{array}{c}0.99 \\
(0.03)\end{array}$ & $\begin{array}{c}0.99 \\
(0.03)\end{array}$ & $\begin{array}{c}1.01 \\
(0.03)\end{array}$ \\
\hline Age & $\begin{array}{c}0.98 \\
(0.02) \\
\end{array}$ & $\begin{array}{c}0.98 \\
(0.02) \\
\end{array}$ & $\begin{array}{c}0.98 \\
(0.02) \\
\end{array}$ & $\begin{array}{c}0.98 \\
(0.02) \\
\end{array}$ & $\begin{array}{r}0.98 \\
(0.02) \\
\end{array}$ \\
\hline HIV Status & $\begin{array}{r}1.67 * * \\
(0.29)\end{array}$ & $\begin{array}{r}1.65 * * \\
(0.29)\end{array}$ & $\begin{array}{c}1.67 * * \\
(0.30)\end{array}$ & $\begin{array}{r}1.73 * * \\
(0.32)\end{array}$ & $\begin{array}{r}1.71 * * \\
(0.32)\end{array}$ \\
\hline Current Partner & $\begin{array}{l}2.05^{*} \\
(0.67) \\
\end{array}$ & $\begin{array}{r}1.83^{\dagger} \\
(0.61) \\
\end{array}$ & $\begin{array}{l}2.05^{*} \\
(0.68) \\
\end{array}$ & $\begin{array}{l}2.34^{*} \\
(0.81) \\
\end{array}$ & $\begin{array}{l}2.08 * \\
(0.74) \\
\end{array}$ \\
\hline Education & $\begin{array}{c}1.21 \\
(0.24)\end{array}$ & $\begin{array}{c}1.12 \\
(0.23)\end{array}$ & $\begin{array}{c}1.20 \\
(0.24)\end{array}$ & $\begin{array}{c}1.15 \\
(0.24)\end{array}$ & $\begin{array}{r}1.07 \\
(0.23) \\
\end{array}$ \\
\hline $\begin{array}{l}\text { Currently } \\
\text { Employed }\end{array}$ & $\begin{array}{c}1.43 \\
(0.53) \\
\end{array}$ & $\begin{array}{c}1.53 \\
(0.58) \\
\end{array}$ & $\begin{array}{c}1.44 \\
(0.54) \\
\end{array}$ & $\begin{array}{c}1.45 \\
(0.55) \\
\end{array}$ & $\begin{array}{r}1.55 \\
(0.61) \\
\end{array}$ \\
\hline Current Income & $\begin{array}{c}0.69 \\
(0.17) \\
\end{array}$ & $\begin{array}{r}0.65^{\dagger} \\
(0.16) \\
\end{array}$ & $\begin{array}{c}0.68 \\
(0.17) \\
\end{array}$ & $\begin{array}{c}0.66 \\
(0.17) \\
\end{array}$ & $\begin{array}{r}0.63 \\
(0.16) \\
\end{array}$ \\
\hline Alcohol Use & $\begin{array}{r}1.31^{\dagger} \\
(0.20) \\
\end{array}$ & $\begin{array}{r}1.30^{\dagger} \\
(0.20) \\
\end{array}$ & $\begin{array}{r}1.32^{\dagger} \\
(0.65) \\
\end{array}$ & $\begin{array}{r}1.34^{\dagger} \\
(0.21) \\
\end{array}$ & $\begin{array}{r}1.32^{\dagger} \\
(0.21) \\
\end{array}$ \\
\hline Substance Use & $\begin{array}{l}1.18^{*} \\
(0.10)\end{array}$ & $\begin{array}{l}1.17^{*} \\
(0.10)\end{array}$ & $\begin{array}{l}1.17^{*} \\
(0.10) \\
\end{array}$ & $\begin{array}{l}1.20^{*} \\
(0.11) \\
\end{array}$ & $\begin{array}{l}1.20 * \\
(0.11)\end{array}$ \\
\hline Social Support & & $\begin{array}{l}1.02 * \\
(0.01)\end{array}$ & & & $\begin{array}{l}1.02 * \\
(0.01)\end{array}$ \\
\hline LGBT Identity & & & $\begin{array}{c}1.09 \\
(0.24) \\
\end{array}$ & & $\begin{array}{c}0.93 \\
(0.23) \\
\end{array}$ \\
\hline $\begin{array}{l}\text { Native Identity } \\
\text { (Actualization) }\end{array}$ & & & $\begin{array}{c}1.05 \\
(0.38) \\
\end{array}$ & & $\begin{array}{c}0.90 \\
(0.37) \\
\end{array}$ \\
\hline $\begin{array}{l}\text { LGBT } \\
\text { Participation }\end{array}$ & & & & $\begin{array}{l}0.80^{*} \\
(0.09) \\
\end{array}$ & $\begin{array}{r}0.82^{\dagger} \\
(0.09) \\
\end{array}$ \\
\hline $\begin{array}{l}\text { Native } \\
\text { Participation }\end{array}$ & & & & $\begin{array}{c}0.99 \\
(0.12) \\
\end{array}$ & $\begin{array}{c}0.96 \\
(0.12) \\
\end{array}$ \\
\hline Constant & 0.22 & 0.08 & 0.13 & 0.73 & 0.41 \\
\hline $\mathrm{F}$ & $2.44 * *$ & $2.48 * *$ & $2.03 *$ & $2.26 * *$ & $1.96^{*}$ \\
\hline
\end{tabular}

Note: Logistic regression odds ratios with standard errors in parentheses.

${ }^{\dagger} \mathrm{p} \leq .1 * \mathrm{p} \leq .05 * * \mathrm{p} \leq .01 * * * \mathrm{p} \leq .001$ (Two-Sided Test) 
Research Question 4.2: Do social support, racial and sexual identity, and racial and sexual community participation mediate the effect of discrimination on depressive symptoms, and if so, to what extent?

The results from model 7 in table 10 indicate that social support is negatively associated with depressive symptoms $(b=-0.07, \mathrm{SE}=0.02, \mathrm{p} \leq .01)$. This result means that those reporting higher levels of social support also report fewer depressive symptoms. Even though the association between heterosexist discrimination and depressive symptoms is not significant $(b=-0.33, \mathrm{SE}=0.63, \mathrm{~ns})$ after adjusting for social support, the findings indicate that higher levels of reported heterosexist discrimination are associated with fewer depressive symptoms. This finding should be interpreted with caution. Model 7 indicates that after adjusting for social support, the association between racial discrimination and depressive symptoms remains significant $(b=1.04, \mathrm{SE}=0.50, \mathrm{p} \leq .05)$, though slightly mediated when compared to model 6. Adjusting for social support has a mediating effect on the association between education and depressive symptoms $(b=0.97, \mathrm{SE}$ $=-0.77, \mathrm{~ns}$ ) meaning that adjusting for social support explains away the relationship between education and depressive symptoms. Model 6 also indicates that the associations between HIV status and depressive symptoms $(b=0.82, \mathrm{SE}=0.45, \mathrm{p} \leq$ $.10)$, employment status and depressive symptoms $(\mathrm{b}=-1.90, \mathrm{SE}=0.91, \mathrm{p} \leq .05)$, and alcohol use and depressive symptoms $(\mathrm{b}=0.86, \mathrm{SE}=0.35, \mathrm{p} \leq .05)$ are all more robust after adjusting for social support, indicating that social support is a suppressor. Though only approaching significance, the association between substance use and depressive symptoms suggests that those who engage in polysubstance use may report more depressive symptoms. 
The results from model 8 in table 10 indicate that LGBT identity is negatively associated with depressive symptoms $(b=-0.03, \mathrm{SE}=0.50, \mathrm{~ns})$. This result indicates higher levels of LGBT identity are associated with fewer depressive symptoms reported in the past week. However this association is not statistically significant and should be interpreted with caution. This model also indicates that Native identity is negative associated with depressive symptoms $(b=-2.11, \mathrm{SE}=0.92, \mathrm{p} \leq$ $.05)$, meaning that higher levels of Native identity are associated with fewer depressive symptoms reported in the past week. Even though the association between heterosexist discrimination and depressive symptoms is not significant (b $=-0.37, \mathrm{SE}=0.64, \mathrm{~ns}$ ) after adjusting for LGBT and Native identity, the findings continue to indicate a negative association as represented in models 6 and 7 , suggesting that higher levels of heterosexist discrimination are associated with fewer depressive symptoms reported in the past week. Model 8 indicates that after adjusting for LGBT and Native identity, the association between racial discrimination and depressive symptoms remains significant $(b=1.36, S E=0.53, p$ $\leq .05)$ compared to model 6 , the adjustment for LGBT and Native identity has a suppression effect on the association between racial discrimination and depressive symptoms indicating that LGBT and Native identity account for some of the variance that allows for a more robust relationship to be shown. Model 8 indicates that adjusting for LGBT and Native identity mediates some of the association between education and depressive symptoms $(b=-1.06, \mathrm{SE}=0.50, \mathrm{p} \leq .05)$ when compared to model 6. This means that adjusting for LGBT and Native explains away the relationship between education and depressive symptoms. Model 8 also indicates 
that the associations between employment status and depressive symptoms $(b=-$ $1.93, \mathrm{SE}=0.92, \mathrm{p} \leq .05)$ and alcohol use and depressive symptoms $(\mathrm{b}=0.86, \mathrm{SE}=$ $0.36, \mathrm{p} \leq .05$ ) are both more robust after adjusting for LGBT and Native identity, indicating that these cultural buffers are suppressors for these associations. Though only approaching significance, the association between substance use and depressive symptoms $(b=0.39, \mathrm{SE}=0.22, \mathrm{p} \leq .10)$ suggests that those who engage in polysubstance use may report higher levels of depressive symptoms.

The results from model 9 in table 10 indicate that LGBT participation $(b=$ $0.10, \mathrm{SE}=0.28, \mathrm{~ns})$ and Native participation $(\mathrm{b}=0.13, \mathrm{SE}=0.31, \mathrm{~ns})$ are not significantly associated with depressive symptoms. Even though the association between heterosexist discrimination and depressive symptoms is not significant $(b$ $=-0.08, \mathrm{SE}=0.65, \mathrm{~ns}$ ) after adjusting for LGBT and Native participation, the finding shows some mediating effect compared to model 6 and a negative association as represented in previous models. Model 9 indicates that after adjusting for LGBT and Native participation, the association between racial discrimination and depressive symptoms remains significant $(\mathrm{b}=1.17, \mathrm{SE}=0.53, \mathrm{p} \leq .05)$. Though compared to model 6, the adjustment for LGBT and Native participation has a suppression effect on the association between racial discrimination and depressive symptoms indicating that these cultural buffers account for some of the variance that allows for a more robust relationship to be shown. This model also indicates a negative association between current partner status and depressive symptoms $(b=-1.72$, SE $=0.85, \mathrm{p} \leq .05)$, meaning that those that reported having a partner indicated that experienced on average 1.72 fewer depressive symptoms than those that reported 
being single. Model 9 indicates that adjusting for LGBT and Native participation mediates the association between education and depressive symptoms $(b=-1.78$, $\mathrm{SE}=0.93, \mathrm{p} \leq .10$ ) when compared to model 6 , as well as some of the association between alcohol use and depressive symptoms $(b=0.80, S E=0.37, p \leq .05)$. This means that adjusting for LGBT and Native explains away the relationship between education and depressive symptoms and some of the association between alcohol use and depressive symptoms. Though only approaching significance, the association between substance use and depressive symptoms $(b=0.38, S E=0.22, p$ $\leq .10)$ suggests that those who engage in polysubstance use may report more depressive symptoms.

Model 10 in table 10 adjusts for all 5 cultural buffers, the results indicate that social support $(\mathrm{OR}=-0.07, \mathrm{SE}=0.02, \mathrm{p} \leq .01)$ is negatively associated with depressive symptoms, meaning higher levels of social support are associated with fewer depressive symptoms reported in the past week. All other cultural buffers are not associated with depressive symptoms. The association between heterosexist discrimination and depressive symptoms is not significant $(b=-0.31, \mathrm{SE}=0.64, \mathrm{~ns})$ after adjusting for all cultural buffers, the finding shows some suppression effect compared to model 6 and a negative association as represented in previous models. Model 10 indicates that after adjusting for cultural buffers, the association between racial discrimination and depressive symptoms remains significant $(\mathrm{b}=1.33, \mathrm{SE}=$ $0.53, \mathrm{p} \leq .05)$. Though compared to model 6 , the adjustment for all cultural buffers has a suppression effect on the association between racial discrimination and depressive symptoms indicating that these cultural buffers account for some of the 
variance that allows for a more robust relationship to be shown. Though only approaching significance, HIV status may be associated with depressive symptoms $(b=0.83, S E=0.46, p \leq .10)$, though this finding should be interpreted with caution. The final model also indicates a mediating effect for the association between education and depressive symptoms $(b=-0.76, \mathrm{SE}=0.51, \mathrm{~ns})$ when compared to model 6 , suggesting that the cultural buffers explain away that relationship. Alcohol use remains significantly associated with depressive symptoms in this model $(b=0.85, \mathrm{SE}=0.36, \mathrm{p} \leq .05)$. Though only approaching significance, the association between substance use and depressive symptoms $(\mathrm{b}=$ $0.36, \mathrm{SE}=0.21, \mathrm{p} \leq .10$ ) suggests that those who engage in polysubstance use may report more depressive symptoms. Overall, when adjusting for all of the cultural buffers, the association between heterosexist discrimination and depressive symptoms is not significant, however the association between racial discrimination and depressive symptoms is more robust. As a result, those reporting higher levels of depressive symptoms after adjusting for the collective of cultural buffers are those who report higher levels of racial discrimination, are not currently employed, drank within the past week, and those who report lower levels of social support. 
Table 10. Linear Regression Models of Native Men who have Sex with Men, Mediating Effects of Cultural Buffers on the Association between Heterosexist and Racial Discrimination on Depressive Symptoms: The HONOR Project, 2005-2007 $(\mathrm{N}=221)$

\begin{tabular}{|c|c|c|c|c|c|}
\hline Variable & M6 & M7 & M8 & M9 & M10 \\
\hline $\begin{array}{l}\text { Heterosexist } \\
\text { Discrimination }\end{array}$ & $\begin{array}{l}-0.26 \\
(0.64)\end{array}$ & $\begin{array}{l}-0.33 \\
(0.63)\end{array}$ & $\begin{array}{l}-0.37 \\
(0.64)\end{array}$ & $\begin{array}{l}-0.08 \\
(0.65)\end{array}$ & $\begin{array}{l}-0.31 \\
(0.64)\end{array}$ \\
\hline $\begin{array}{l}\text { Racial } \\
\text { Discrimination }\end{array}$ & $\begin{array}{l}1.13^{*} \\
(0.51)\end{array}$ & $\begin{array}{l}1.04^{*} \\
(0.50)\end{array}$ & $\begin{array}{l}1.36^{*} \\
(0.53)\end{array}$ & $\begin{array}{l}1.17^{*} \\
(0.53)\end{array}$ & $\begin{array}{l}1.33^{*} \\
(0.53)\end{array}$ \\
\hline Age & $\begin{array}{c}0.06 \\
(0.04)\end{array}$ & $\begin{array}{r}0.05 \\
(0.04)\end{array}$ & $\begin{array}{r}0.07^{\dagger} \\
(0.04)\end{array}$ & $\begin{array}{r}0.06 \\
(0.04)\end{array}$ & $\begin{array}{c}0.05 \\
(0.04)\end{array}$ \\
\hline HIV Status & $\begin{array}{c}0.74 \\
(0.46)\end{array}$ & $\begin{array}{r}0.82^{\dagger} \\
(0.45)\end{array}$ & $\begin{array}{r}0.74 \\
(0.46) \\
\end{array}$ & $\begin{array}{r}0.76 \\
(0.47) \\
\end{array}$ & $\begin{array}{c}0.83^{\dagger} \\
(0.46)\end{array}$ \\
\hline Current Partner & $\begin{array}{l}-1.56^{\dagger} \\
(0.85) \\
\end{array}$ & $\begin{array}{l}-0.97 \\
(0.84) \\
\end{array}$ & $\begin{array}{l}-1.44^{\dagger} \\
(0.84) \\
\end{array}$ & $\begin{array}{l}-1.72 * \\
(0.85) \\
\end{array}$ & $\begin{array}{l}-1.05 \\
(0.86) \\
\end{array}$ \\
\hline Education & $\begin{array}{l}-1.15 * \\
(0.50)\end{array}$ & $\begin{array}{l}-0.77 \\
(0.50) \\
\end{array}$ & $\begin{array}{l}-1.06^{*} \\
(0.50)\end{array}$ & $\begin{array}{l}-1.14^{*} \\
(0.52)\end{array}$ & $\begin{array}{l}-0.76 \\
(0.51)\end{array}$ \\
\hline $\begin{array}{l}\text { Currently } \\
\text { Employed }\end{array}$ & $\begin{array}{l}-1.85^{*} \\
(0.93)\end{array}$ & $\begin{array}{l}-1.90^{*} \\
(0.91)\end{array}$ & $\begin{array}{l}-1.93^{*} \\
(0.92)\end{array}$ & $\begin{array}{l}-1.78^{\dagger} \\
(0.93)\end{array}$ & $\begin{array}{l}-1.89 * \\
(0.91)\end{array}$ \\
\hline Current Income & $\begin{array}{l}-0.90 \\
(0.60) \\
\end{array}$ & $\begin{array}{l}-0.62 \\
(0.59) \\
\end{array}$ & $\begin{array}{l}-0.84 \\
(0.60) \\
\end{array}$ & $\begin{array}{l}-0.86 \\
(0.60) \\
\end{array}$ & $\begin{array}{l}-0.65 \\
(0.60) \\
\end{array}$ \\
\hline Alcohol Use & $\begin{array}{c}0.84^{*} \\
(0.36) \\
\end{array}$ & $\begin{array}{r}0.86^{*} \\
(0.35) \\
\end{array}$ & $\begin{array}{r}0.86^{*} \\
(0.36) \\
\end{array}$ & $\begin{array}{r}0.80^{*} \\
(0.37) \\
\end{array}$ & $\begin{array}{l}0.85^{*} \\
(0.36) \\
\end{array}$ \\
\hline Substance Use & $\begin{array}{r}0.40^{\dagger} \\
(0.22)\end{array}$ & $\begin{array}{r}0.40^{\dagger} \\
(0.21)\end{array}$ & $\begin{array}{r}0.39^{\dagger} \\
(0.22)\end{array}$ & $\begin{array}{r}0.38^{\dagger} \\
(0.22) \\
\end{array}$ & $\begin{array}{l}0.36^{\dagger} \\
(0.21)\end{array}$ \\
\hline Social Support & & $\begin{array}{c}-0.07 * * \\
(0.02)\end{array}$ & & & $\begin{array}{c}-0.07 * * \\
(0.02)\end{array}$ \\
\hline LGBT Identity & & & $\begin{array}{l}-0.03 \\
(0.50) \\
\end{array}$ & & $\begin{array}{r}0.38 \\
(0.53) \\
\end{array}$ \\
\hline $\begin{array}{l}\text { Native Identity } \\
\text { (Actualization) }\end{array}$ & & & $\begin{array}{l}-2.11 * \\
(0.92)\end{array}$ & & $\begin{array}{l}-1.44 \\
(0.93) \\
\end{array}$ \\
\hline $\begin{array}{l}\text { LGBT } \\
\text { Participation }\end{array}$ & & & & $\begin{array}{l}0.10 \\
(0.28) \\
\end{array}$ & $\begin{array}{c}0.01 \\
(0.28) \\
\end{array}$ \\
\hline $\begin{array}{l}\text { Native } \\
\text { Participation }\end{array}$ & & & & $\begin{array}{l}0.13 \\
(0.31) \\
\end{array}$ & $\begin{array}{r}0.02 \\
(0.33) \\
\end{array}$ \\
\hline Constant & 7.60 & 10.54 & 14.43 & 6.21 & 12.15 \\
\hline $\mathrm{F}$ & $6.27 * * *$ & $7.03 * * *$ & $5.74 * * *$ & $5.36 * * *$ & $5.39 * * *$ \\
\hline
\end{tabular}

Note: Linear regression coefficients with standard errors in parentheses. ${ }^{\dagger} \mathrm{p} \leq .1 * \mathrm{p} \leq .05 * * \mathrm{p} \leq .01 * * * \mathrm{p} \leq .001$ (Two-Sided Test) 
Research Question 4.3: Do social support, racial and sexual identity, and racial and sexual community participation mediate the effect of depressive symptoms on HIV risk behaviors, and if so, to what extent?

The results of model 8 shown in table 8 indicate that the odds ratio for depressive symptoms is not significantly associated with unprotected anal intercourse (OR $=0.98, \mathrm{SE}=0.03, \mathrm{~ns})$. After adjusting for social support in model 9, the odds ratio for depressive symptoms is $1.00(\mathrm{OR}=1.00, \mathrm{SE}=0.02, \mathrm{~ns})$. After adjusting for LGBT and Native identity in model 10, the odds ratio for depressive symptoms $(\mathrm{OR}=0.99, \mathrm{SE}=0.03, \mathrm{~ns})$ is below 1.00 . The odds ratio remains constant from model 10 to model 11 that adjusts for LGBT and Native participation (OR = $0.99, \mathrm{SE}=0.03, \mathrm{~ns}$ ). In the final model adjusting for all 5 cultural buffers, the odds ratio for depressive symptoms $(\mathrm{OR}=1.01, \mathrm{SE}=0.03, \mathrm{~ns})$ is slightly larger than 1.00 .

The results of model 8 shown in table 9 indicate that the odds ratio for depressive symptoms is not significantly associated with unprotected receptive anal intercourse (OR $=0.99, \mathrm{SE}=0.03, \mathrm{~ns})$. After adjusting for social support in model 9 , the odds ratio for depressive symptoms is $1.01(\mathrm{OR}=1.01, \mathrm{SE}=0.03, \mathrm{~ns})$. After adjusting for LGBT and Native identity in model 10, the odds ratio for depressive symptoms $(\mathrm{OR}=0.99, \mathrm{SE}=0.03, \mathrm{~ns})$ is below 1.00 . The odds ratio remains constant from model 10 to model 11 that adjusts for LGBT and Native participation respectively $(\mathrm{OR}=0.99, \mathrm{SE}=0.03, \mathrm{~ns})$. In the final model that adjusts for all 5 cultural buffers, the odds ratio for depressive symptoms $(\mathrm{OR}=1.01, \mathrm{SE}=0.03, \mathrm{~ns})$ is slightly larger than 1.00 . 


\section{CHAPTER 6: DISCUSSION}

This study sought to examine the relationship between discrimination based on both race and sexual orientation, alone and in combination, and HIV risk behavior among a national sample of Native MSM, a population with dual minority status that has been underrepresented in the literature. Additionally this study examined whether the relationship between discrimination and HIV risk behavior is mediated by depressive symptoms. Lastly this study sought to examine whether the relationships between discrimination, depressive symptoms, and HIV risk behavior were mediated by social support, LGBT and Native identity, and LGBT and Native community participation. The findings from this study showed some but not all of the hypothesized associations between discrimination, depression, and HIV risk behaviors. The following discussion section highlights and addresses the study findings with attention to implications for policy and research.

Before discussing the findings by specific aim, it is important to discuss the sample of Native MSM in relation to the literature of other racial minority MSM. Compared to samples of Asian, Black, Latino, and Pacific Islander MSM (Ayala et al., 2012; Bruce et al., 2008; Chae \& Yoshikawa, 2008; Chen \& Tryon, 2012; Choi et al., 2013; Díaz et al., 2001; Diaz et al., 2004; Jarama et al., 2005; Reisner et al., 2010; Hirokazu Yoshikawa et al., 2004), this sample was slightly older, less likely to have a partner, reported higher unemployment despite comparable levels of education, had lower income, and comparable levels of self-reported HIV status (with $36 \%$ of the sample self-reporting an HIV positive status). The sample from the current study 
reported relatively high rates of unprotected anal intercourse (UAI). In the past 12 months, $39.4 \%$ of respondents reported at least one episode of UAI and 32.6\% reported at least one episode of unprotected receptive anal intercourse (URAI). These figures are similar to recent findings in other studies of racial minority MSM (Chae \& Yoshikawa, 2008; Jarama et al., 2005; Hirokazu Yoshikawa et al., 2004). Though similar to other racial minority MSM populations, it is important restate the wide diversity among Native populations, and specifically among Native MSM, with respect to demographic characteristics, cultural values, and socioeconomic circumstances. Given such diversity, Native MSM may have different views toward sexual behavior and different experiences as racial and sexual minorities, particularly given the existence of the concept and history of being two-spirit (Han, 2007, 2008).

\section{DISCRIMINATION AND HIV RISK BEHAVIOR}

The first aim was to examine whether or not racial and heterosexist discrimination were associated with HIV risk behaviors. The findings indicate that exposure to heterosexist discrimination is associated with UAI, whereas racial discrimination is not. Consistent with some of the literature, particularly among Asian MSM (Chae \& Yoshikawa, 2008; Jarama et al., 2005; Hirokazu Yoshikawa et al., 2004), the current study finds that racial discrimination is not associated with UAI. Of the explanations, Chae and Yoshikawa (2008) found that other forms of discrimination, specifically anti-immigrant discrimination, have strong associations with UAI among Asian MSM. Similarly, Jarama and colleagues (2005) found that 
heterosexist discrimination and not racial discrimination is associated with UAI among Latino MSM and suggest that experiences of physical, verbal, and emotional abuse regarding one's same sex behavior and feelings negatively impact one's self esteem while contributing to increased feelings of shame and denial. However, Jarama and colleagues do not indicate the source of abuse. It is also important to note that there was a lack of variation in the racial discrimination variable. That is to say, that racial discrimination may be associated with UAI, but that association was not found in the current study given the lack of variation of distress related to racial discrimination among this sample. Moreover, the current study used microaggressions, a subtle form of discrimination, to assess racial discrimination. It is plausible that a different type of racial discrimination, overt forms of discrimination specifically, may be associated with UAI.

Given that racial discrimination is not associated with UAI and that URAI is a form of UAI, it is not surprising that racial discrimination is not associated with URAI. The finding that racial discrimination is not associated with URAI does not suggest that racial discrimination is not associated with sexual positioning among Native MSM. That is to say that within this sample of Native MSM there are other more relevant factors associated with URAI aside from racial discrimination, such as demographic factors such as HIV status and having a main partner. The latter finding provides some support for the literature indicating that MSM engage in URAI with their main partners (Davidovich et al., 2006; De Vroome et al., 2000; McLean et al., 1994; McNeal, 1997), though the intent of this behavior such as demonstration of love and commitment or the inability to negotiate condom use cannot be 
determined from these data. Additionally, the association of substance use and URAI suggests that this sample of Native MSM may be engaging in URAI as a result of diminished inhibitions. Despite the high exposures to racial discrimination reported in this sample, these findings indicate that Native MSM engaging in URAI may be contending with other social determinants with respect to using or not using condoms.

Unlike racial discrimination, this study shows that heterosexist discrimination is associated with UAI. This finding is consistent with some of the literature (Ayala et al., 2012; Diaz et al., 2004; Mizuno et al., 2012). Furthermore, the findings in this study suggest a suppressing effect of all other variables, including racial discrimination. That is to say that when additional variables are added to each regression model, the predictive validity of heterosexist discrimination increases as the adjusting variables clear out the variance allowing for a more true and robust association to be shown.

The explanations for why heterosexist discrimination predicts UAI are unclear given that the mechanisms, which include the sources, types of discrimination, and the frequency at which discrimination occurs, are not well understood. Of the explanations provided, Diaz and colleagues (2004) suggest that MSM experiencing higher levels of discrimination may feel less empowered or may internalize negative messages about themselves and / or their behavior and thus fail to protect themselves. As a result, MSM experiencing discrimination may have fewer coping skills, self-esteem, and confidence to stand up to difficult situations and negotiate condom use with a dominant partner. Alternatively they may engage in 
the high risk behavior (UAI) to feel invincible against the barrage of mistreatment and harassment and "otherness" experienced on a daily basis.

However, prior research has investigated this association with the ability to control for behavior among main and casual partners as well as those in monogamous sexual relationships (Ayala et al., 2012; Chae \& Yoshikawa, 2008; Jarama et al., 2005; Mizuno et al., 2012). One explanation for including these factors is that MSM could be in monogamous relationships in which the perceived risk of transmitting or acquiring HIV is extremely low or perceived as nonexistent. Another explanation is that MSM may engage in unprotected sex with their main partner and protected sex with their casual partner(s). Both explanations indicate that MSM are behaving in ways that demonstrate they are empowered and making decisions surrounding their sexual health behavior. That is to say that being in a monogamous sexual relationship or strictly using condoms with causal partners are indicators that MSM may be engaging in unprotected sex at least once in the past twelve months despite the fact that HIV prevention does influence their behavior.

Taking these findings together, one explanation of why heterosexist discrimination was found to be associated with UAI and not racial discrimination is about socialization and specifically the aspects of when Native MSM are socialized and who contributes to their socialization. Within an indigenous perspective that incorporates the relevant historical, political, and social context, Native men that grow up to be Native MSM (referred to as "young Native MSM" going forward) are socialized early in life about how dominant culture views Natives and learn early in life what it means to be a racial minority. Additionally, most (not all, but most) have 
other Native people to socialize them to traditional ways of resisting the negative messages about being a racial minority. The socialization process of resistance or resiliency has been taught to young Native MSM for more than half a millennium and through time periods that included federal policies of extermination, assimilation, termination, and acculturation. Despite all that dominant culture has taught young Native MSM over the years about being a racial minority, there has always been positive socialization processes about being Native by other Natives (Mead, 1996). This is how Native people have learned to survive and cope with racial discrimination (Smith, 2010). Given these aspects of socialization and the overall high level of reported racial discrimination, I posit that Native MSM either experience less discrimination from with the larger MSM community and / or they have developed resilient ways of coping with racial discrimination that do not diminish their capacity to negotiate condom use.

However, the socialization process of resistance or resiliency cannot be said of young Native MSM with regard to being a sexual minority and distress from heterosexist discrimination. With respect to heterosexist discrimination, young Native MSM develop their sexual minority identity through socialization processes that, according to Erving Goffman, generate stigma. Goffman suggests that those with power (what he refers to as the "majorities") socialize the minorities. During the socialization process minorities learn and internalize the beliefs of the majorities and as a result internalize a sense of failure and abnormality about themselves for being minorities (Goffman, 1963). Goffman's ideas of socialization are also seen in previous research among Latino MSM. For example, Diaz and 
colleagues (2001), found 3 most common experiences of young Latino MSM during childhood were hearing that gays are not normal people (91\%), hearing that gay people grow up to be alone (71\%), and having a deep feeling that the respondent's homosexuality hurt and embarrassed his family (70\%). Additionally, Diaz and colleagues reported that the majority of men (64\%) reported having to pretend to be straight at some point in their adult lives and $29 \%$ reported that they had to move away from family or friends to live their homosexual lives. Furthermore, this idea of socialization is also found in literature among Native sexual minorities. As mentioned previously, Western colonization and Christianization attacked traditional Native conceptions of sexual orientation and identity, undermined traditional ceremonial and social roles for Native MSM, and replaced traditions of acceptance and inclusivity with shaming and condemnation (Tinker, 1993).

As such, young Native MSM are socialized under circumstances that are similar to what Goffman theorizes as the socialization of minorities by the majorities. So when young Native MSM are socialized early in life about how dominant culture views MSM and learn early in life what it means to be a sexual minority, they are less likely to have support from a community of sexual minorities or allies. It isn't until these men are adults that most of them seek out a community of MSM and or Native MSM to garner that support and socialization about being a sexual minority. Given the socialization, potential lack of support growing up, and the overall high level of reported heterosexist discrimination, I posit that Native MSM experience heterosexism from the larger heteronormative population, which can include other Natives, and / or they have a delay in developing forms of 
resiliency that leads to diminished capacity to negotiate condom use. Lastly, it is probable that Native MSM despite experiences of heterosexist discrimination may lead lives empowered to make decisions surround their sexual health that incorporate limited or no condom use. Those factors were not analyzed directly in this study.

Again, the mechanisms that help explain the direct association of heterosexist discrimination and HIV risk remain unclear. Additional research regarding the specific types, sources, and frequency of discrimination with detailed nuances of condom use should be explored among racial minority MSM and specifically Native MSM.

\section{DISCRIMINATION AND DEPRESSION}

The second aim was to examine whether or not racial and heterosexist discrimination were associated with depressive symptoms as a means of testing the minority stress model. The levels of depressive symptoms were high in this sample, with an average CES-D score of 10.53 and $55.4 \%$ reporting scores at or above 10, which is considered the clinical cutoff for probable depression. These figures are substantially higher than for White MSM (Perdue et al., 2003; 22\% of that sample scored above the cutoff). They are comparable to those in prior studies of Asian MSM (Chae \& Yoshikawa, 2008; 41\% scored above the cutoff) and Black MSM (Peterson et al., 1996; 50\% of that sample scored above the cutoff). The findings indicate that exposure to racial discrimination predicted depressive symptoms, whereas heterosexist discrimination did not. 
Experiences of racial discrimination were associated with higher levels of depressive symptoms, suggesting that racial discrimination may affect the mental health and overall well being of Native MSM. Prior research has documented the significant association between racism and depression among U.S. Asian and Pacific Islander and Latino MSM (Chae \& Yoshikawa, 2008; Díaz et al., 2001; Diaz et al., 2004; Hirokazu Yoshikawa et al., 2004) and most recently in a sample that included Asian and Pacific Islander, Black, and Latino MSM (Choi et al., 2013). Although I controlled for potential covariates of depressive symptoms (e.g. age, HIV status, current partner status, socioeconomic status indicators, and alcohol and substance use), the mechanism of racial discrimination (e.g. sources and frequency) was not captured and should be explored in future studies.

In contrast, experiences of heterosexist discrimination were not associated with higher levels of depressive symptoms. This finding contradicts much of the literature on discrimination and depression among racial minority MSM (Ayala et al., 2012; Choi et al., 2013; Mizuno et al., 2012; Hirokazu Yoshikawa et al., 2004). Potential reasons for finding no association between heterosexist discrimination and depressive symptoms are two-fold. First, the issue of temporality may confound this analysis, meaning that measures of discrimination were asked over a lifetime, whereas depressive symptoms were asked over the prior week. As such it is possible that Native MSM may have experienced heterosexist discrimination at some point in their lifetime but not recently enough to have an impact on their mental health within the past week. The second potential explanation for the lack of association is that Native MSM may have organized their lives in such a way as to 
avoid heterosexist discrimination. Given the previous explanations on young Native MSM socialized on how dominant culture views sexual minorities it is possible that many Native MSM may experience heterosexist discrimination during adolescence and early adulthood. This sample of Native MSM is close to 40 years of age and it is common that at this time in life, many MSM, specifically gay and bisexual identified men, have organized their life in a way to avoid many of the daily hassles of being a sexual minority (e.g. move to an urban center, live in a "gayborhood" or "gay ghettos," socialize with a supportive network of family and friends) (Mills et al., 2001; Yoshikawa, Wilson, \& Chae, 2002; Yoshikawa et al., 2004). It is possible then when asking Native MSM to account for their lifetime experience with heterosexist discrimination they included their experiences growing up. However, these experiences occurred so long ago that they may not have influence on depressive symptoms experienced within the past week, creating an issue of temporality.

Though heterosexist discrimination may have occurred earlier in life as posited above, racial discrimination and economic hardship may have occurred more recently and as such be more influential indicators of the current mental health of this sample. It could be that those reporting racial discrimination have had recent experiences of racial discrimination. Similarly, the level of education in conjunction with the high unemployment rate could have elevated depressive symptoms due to financial hardship and severe unemployment, which were assessed within a similar timeframe and significantly associated with depressive symptoms. This is to say that depressive symptoms are associated with experiences of what happened within the timing of participating in the HONOR project and that 
heterosexism may not have occurred within the timeframe whereas experiences of racism and economic hardship may have occurred within that timeframe.

\section{DEPRESSION AND HIV RISK BEHAVIOR}

The third aim was designed to examine whether depressive symptoms were associated with HIV risk behaviors as a means of testing whether or not Native MSM used HIV risk behaviors as a maladaptive coping strategy. The findings from this study indicate that depressive symptoms are not associated with either UAI or URAI. These findings are consistent with studies of Asian MSM (Yoshikawa et al., 2004) and inconsistent among studies of Black MSM (Crawford et al., 2002; Myers et al., 2003; Reisner et al., 2009).

Though these findings do not indicate that depressive symptoms are associated with UAI or URAI, they do clearly indicate that alcohol use is consistently associated with UAI and that substance use is associated with URAI. Furthermore, alcohol use is associated with depressive symptoms and substance use is approaching a statistically significant relationship with depressive symptoms. These findings taken together suggest that alcohol use, and to a lesser extent substance use, may be a better mechanism to use when investigating the indirect association of discrimination and HIV risk behaviors. The associations of HIV status and substance use with UAI suggest that those with HIV and those with a partner are at higher odds of engaging in URAI than those who are HIV negative and without a partner. Though this provides some support for serosorting behavior, the fact remains that these behaviors are not coping processes related to depressive symptoms. 


\section{INFLUENCES OF CULTURAL DETERMINANTS}

The fourth aim was designed to examine whether or not social support, racial and sexual identity, and racial and sexual community participation mediated the associations from the first three specific aims. Results of the present study indicate that social support was significantly and negatively associated with depressive symptoms, suggesting a protective effect against depressive symptoms.

Additionally, LGBT community participation was significantly and negatively associated with URAI, suggesting a protective effect against URAI. However, none of the cultural determinants fully mediated the associations of specific aims.

The findings from this study with regard to social support showed some but not all of the hypothesized associations between discrimination, depression, and HIV risk behaviors. As found in previous studies, social support was negatively associated with depressive symptoms (Díaz et al., 2001; Meyer, 2003) showing some support for specific aim two. However, social support was not negatively associated with HIV risk outcomes. Instead, social support was found to be a predictor of UAI (trending toward significance in specific aim one) and URAI (significant predictor of URAI in specific aim one), findings that contradicts previous research (Ayala et al., 2012; Vu et al., 2011). Social support, though beneficial in protecting against depressive symptoms, may include support from sexual partners. This is to say that the support Native MSM receive from their main and or causal sex partners. The support garnered from sexual partners may build bonds of trust and safety, which may lessen the levels of depressive symptoms while also facilitating 
unprotected sexual behavior between Native MSM and their partners. The sources and types of social support of Native MSM should be explored in future studies as the evidence from this study suggests that social support has benefits for mental health while simultaneously contributes to HIV condomless sex.

Contrary to my hypotheses, the findings from this study found that racial and sexual identity were not related to any of the hypothesized associations between discrimination, depression, and HIV risk behaviors. This finding contradicts the previous literature with regard to group identity (Branscombe et al., 1999; Chae \& Yoshikawa, 2008; Han, 2008; Romero \& Roberts, 2003; Sellers et al., 2003; Sellers \& Shelton, 2003; Walters, 1999). The results indicate that on average the sample had positive Native identity and fair LGBT identity and the sample had little variation on either measure. This lack of variation makes it difficult to measure the effect of identity. This finding may also be a result of the fact that identity was measured by race and sexuality separately and did not include a measure of two-spirit identity. Unlike other racial minority MSM, Native MSM have a traditional place and role within the larger Native community. Future studies should investigate two-spirit identity in relation to the effect of discrimination on depression and HIV risk behavior.

The findings from this study with regard to racial and sexual community participation showed some but not all of the hypothesized associations between discrimination, depression, and HIV risk behaviors. Participation within the larger Native community was not related to any of the hypothesized associations and fails to support the prior research that states racial minority MSM who are apart of their 
racial community are less likely to engage in unprotected sex (Chng \& Geliga-Vargas, 2000; Han, 2007). This study shows that participating within the LGBT community has a slight protective effect for UAI and a strong protective effect for URAI, which supports prior research (Chng \& Geliga-Vargas, 2000; Ratti et al., 2000; Seibt et al., 1995). Prior research operationalized LGBT community participation by the number of venues one attends or the number of subscriptions to gay magazines. The current study is different than previous studies as it examines the frequency index assessing how often one participates in the community. Using this measure of frequency contributes a more nuanced aspect of the construct of LGBT community participation and allows for a better understanding of the relationship of community participation and health and health behavior. Additional research is warranted on the other nuances (i.e. type, length, and level of participation) of LGBT participation among Native MSM as they related to HIV risk behavior among Native MSM.

Given that only Native participation was not significantly associated with HIV risk behavior, the evidence presented here does not support the enculturation hypothesis. The enculturation hypothesis states that enculturation is a process of community engagement that does not force Native MSM to 'fit' into white definitions of 'gay' or 'Native', rather reclaims a long cultural practice of valuing an identity and practice of being two-spirit thereby increasing self-esteem thereby diminishing depressive symptoms and also serving as a protective factors against risk taking behavior such as UAI and URAI. However, a post-hoc analysis using only two measures assessing community participation specific to the two-spirit community, 
indicates that the odds of engaging in UAI decreased which supports the enculturation hypothesis from an intersectional stand point. Additional research is warranted on the nuances (i.e. type, length, and level of participation) of two-spirit community participation as they relates to HIV risk behavior among Native MSM.

\section{STRENGTHS AND LIMITATIONS}

This study has several limitations that are important to note. First, due to the retrospective cross-sectional design of the study, the directional relationship between racial and heterosexist discrimination and the HIV risk behavior variables cannot be confirmed and as such the data used in this study do not allow for a discussion of causality. Longitudinal investigations are needed to examine the timing of the proposed relationships and confirm a causal sequence (Lehavot et al., 2009).

Second, the respondent-driven sampling method used to recruit study participants may not have resulted in a representative sample of all sexual minority Native men at the study sites. However, the goal for this analysis is to examine the hypothesized associations within this under researched population, and respondent-driven sampling allowed for the recruitment of the largest sample of Native MSM in the United States. Additionally, these data allow for broad definitions of sexuality with the inclusion of sexual orientation designations of two-spirit and heterosexual as well as allowing for sexuality to be defined beyond self-identified sexual orientation to include dimensions of sexuality based on behavior, which has been problematic in previous research (Chae et al., 2010). 
Third, although this sample is likely to be representative of urban two-spirit men, this sample is certainly not representative of the larger two-spirit population as the perspective of rural dwelling two-spirits were not included in the study. This also warrants the concern of combining Native MSM from different geographic areas and tribal affiliations and analyzing them as a single racial group. The small sample size limits the precision of the study estimates. However, these data stem from the largest dataset available to examine the proposed relationships.

Fourth, the findings rely on respondents' self-reported behaviors, which cannot be independently verified. As with other self-reports of sensitive information, the data are subject to the possible influences of social desirability and recall bias. Although computer-assisted self-interviewing was used to reduce inhibitions about disclosing, the accuracy of participants' responses cannot be determined. As such respondents' risk behaviors may have underestimated the true prevalence given that some of these behaviors may be perceived as socially undesirable and that any findings are likely conservative estimates (Cassels et al., 2011). Additionally, respondents may have recall bias of particular events from the past and the fine details of these events may change over time (Lehavot et al., 2009).

Fifth, the measures used in the HONOR project are limited in their ability to address relations based on race and sexual orientation. Most measures of the Microaggression Distress Scale indicated "non-native" as opposed to defining a single race, meaning the race relations from these data are designed to be Native versus all other races, resulting in restrictions on examine specific race relations. Similarly, the two-spirit microaggression scale aggregates sexual minority 
subgroups allowing for only an assessment of sexual minorities versus heterosexuals. This phenomenon may play a factor in why some risk behaviors are more closely associated with one type of discrimination than another (Mizuro et al., 2012).

Lastly, these data do not include all potential explanatory variables when modeling the associations of discrimination, depression, and HIV risk behavior. For example, other studies have examined the importance of linking situations that promote sexual risk-taking in addition to individual, social, and cultural determinants (Wilson, Díaz, Yoshikawa, \& Shrout, 2009).

\section{CONCLUSION}

This study examined the relationship between discrimination and HIV risk behaviors, both directly and indirectly by way of depressive symptoms, among a national sample of Native MSM. With respect to the direct relationship, heterosexist discrimination was associated with both UAI and URAI, whereas racial discrimination was not associated with either UAI or URAI. The investigation into the indirect relationship between discrimination and HIV risk behaviors indicated that racial discrimination, and not heterosexist discrimination, is associated with depressive symptoms and that depressive symptoms are not associated with HIV risk behavior. Furthermore, depressive symptoms is not a mechanism that explains the association between discrimination and HIV risk and perhaps the better mechanisms to examine in future studies is alcohol use and, to a lesser extent, substance use. Additional research is suggested to better understand the 
associations of discrimination and HIV risk behaviors by investigating the types, sources, frequency, and the temporality of discrimination.

The findings of the current study indicate that racial discrimination is associated with depressive symptoms. Additionally, the findings indicate that alcohol use is associated with depressive symptoms as well as UAI. Taken together these findings suggest an indirect relationship between racial discrimination and UAI. That is to say that racial discrimination could be associated with UAI through a combination of depressive symptoms and alcohol use. With respect to heterosexist discrimination, the findings suggest a direction relationship with UAI. Overall, the findings of this study suggest that even though racial and heterosexist discrimination are associated with different outcomes, both forms of discrimination still matter in respect to the health and wellbeing of Native MSM, they just do so differently.

\section{IMPLICATIONS AND FUTURE DIRECTIONS}

Despite these limitations, this study has important implications for mental health and HIV prevention interventions for Native MSM as well as future research with this population. This study provides one of the first insights into the impacts of discrimination on depression and HIV risk behaviors among Native MSM, a group that has been virtually ignored in HIV prevention efforts and the larger academic community. Furthermore, this study supports and recognizes the importance of addressing social and cultural determinants of health in dealing with the HIV 
epidemic (Dean \& Fenton, 2010; Sharpe et al., 2010), and research addressing heterosexism and racism must be part of these endeavors.

The high levels of depressive symptoms, with over half of the sample at risk for clinical diagnosis of depression, are of particular concern. The study's results have several implications for further research and intervention development with respect to specific types, sources, and frequency of discrimination differentially linked to negative mental health outcomes among Native MSM. While the present study did not find evidence that heterosexist discrimination is associated with depressive symptoms; this phenomena along with racial discrimination should be investigated further. These results suggest there is a need to expand the exploration of potential sources, frequency, and timing of stress to reveal the specific mechanisms by which racism and heterosexism may affect mental health. The current recommendation for health providers and interventions is to provide a space for racial minority MSM to gather and discuss their experiences with discrimination and depression. The opportunity to gather, share stories, and find support are thought to improve social support by providing an outlet for releasing stress and creating community among racial minority MSM in which they feel comfortable and empowered to seek out mental health services. Given that Native MSM are small in number compared to their racial minority MSM counterparts, having a space for Native MSM to come together raises some challenges on feasibility and sustainability of such programming. However, providing a space for Native men to gather could be an alternative. This would allow for Native men to share stories and find support as well as to discuss cultural values, which include 
the inclusion of two-spirit people within the larger Native community, while addressing issues of heterosexism within the broader Native community. As such, a gathering of Native men could be a point of entry for structural interventions regarding discrimination.

Evidence-based behavioral interventions to prevent HIV transmission have been designed for Black MSM (Wilton et al., 2009) and Asian and Pacific Islander MSM (Choi et al., 1996). These interventions address both heterosexism and racism through group discussions of how these experiences are related to sexual and substance use risk behaviors (Mizuro et al., 2012) similar to those proposed for mental health interventions. Given that HIV status and partner status were consistently associated with UAI and URAI, intervention research should address the dynamics of male couples in which one or both partners are Native MSM as well as harm reduction approaches and sexual agreements within these partnerships. As of today, however, no evidence-based behavioral interventions are available specifically for Native MSM and given the high prevalence of HIV infection and risk behaviors within this sample, more intervention research is urgently needed for this population.

The results from this study provide evidence that heterosexist discrimination should be addressed in future HIV related research, policy, and interventions with Native MSM. However, additional research is warranted to further determine whether and how different types, sources, and frequency of experiences of discrimination might cause Native MSM to engage in HIV risk behaviors. This research should also pay attention to cultural determinants that might alleviate the 
negative effects of discrimination with special attention given to two-spirit identity, two-spirit roles. Given this sample was drawn from large urban centers, it is important to note that many tribal communities and their respective traditions surrounding two-spirit are represented within an urban context. As such, it is important to identify ways in which the traditions of being two-spirit are understood and enacted and how these understandings and enactments impact mental and behavioral health.

Research needs to also give attention to the cultural determinants relating to LGBT community participation. The measures used for this study assessed frequency of attending bars/night clubs, events, and websites/chat rooms. Though adequate for the time, these measures of community participation are limited in scope and do not account for the surge in social media including mobile applications for connecting with other people as well as other outlets for community participation (such as advocacy and community organizing) that foster community building and a sense of collective identity. The LGBT, Native, and two-spirit cultural determinants shape how these men experience their own lives and the various forms of discrimination they encounter. This research may further inform the interventions and the development of policies and practices that address discrimination at the individual level, by equipping people with resources and skills on how to cope with discrimination personally, as well as at the community level, where structural interventions can aim to reduce discrimination within a society.

The findings from this study, in comparison with prior research on racial minority MSM, illustrate why a one-size-fits-all approach to complex social issues is 
inappropriate and suggest that HIV prevention strategies need to address racism and heterosexism differentially for different racial minority MSM. Innovative prevention programs can focus efforts to the specific needs and cultural characteristics of each population to promote safer sexual practices. It is essential to begin examining HIV infection as a marker of risk factors beyond the individual level and assess the impacts of social and cultural determinants associated with race and sexuality. Framing HIV infection in this way contextualizes Native populations, and Native MSM specifically, in relation to HIV infection as an issue of social justice and aligns it with the legacy of colonization. 


\section{REFERENCES:}

Aggleton, P. (2004). Sexuality, HIV Prevention, Vulnerability and Risk. Journal of Psychology and Human Sexuality, 16(1), 1-11.

Allegretto, S., \& Arthur, M. M. (2001). An Empirical Analysis of Homosexual/Heterosexual Male Earnings Differentials: Unmarried and Unequal? Industrial and Labor Relations Review, 54, 631-646.

Anderson, L. P. (1991). Acculturative Stress: A Theory of Relevance to Black Americans. Clinical Psychology Review, 11, 685-702.

Aneshensel, C. S. (1992). Social Stress: Theory and Research. Annual Review of Sociology, 18, 15-38.

Anguksuar [LaFortune R]. (1997). A postcolonial perspective on Western [mis]conceptions of the cosmos and the restoration of indigenous taxonomies. In S.-E. Jacobs, W. Thomas, \& S. Lang (Eds.), Two-spirit people: Native American gender identity, sexuality, and spirituality (pp. 217-222). Chicago, IL: University of Illinois Press.

Anthias, F. (1990). Race and Class Revisited-Conceptualizing Race and Racisms. Sociological Review, 38, 19-42.

Appleby, P. R., Marks, G., Ayala, A., Miller, L. C., Murphy, S., \& Mansergh, G. (2005). Consideration of Future COnsequences and Unprotected Anal Intercourse Among Men Who Have Sex With Men. Journal of Homosexuality, 50(1), 119-133.

Aral, S. O., Adimora, A. a, \& Fenton, K. a. (2008). Understanding and responding to disparities in HIV and other sexually transmitted infections in African Americans. Lancet, 372(9635), 337-40. doi:10.1016/S0140-6736(08)61118-6

Ayala, G., Bingham, T., Kim, J., Wheeler, D. P., \& Millett, G. a. (2012). Modeling the impact of social discrimination and financial hardship on the sexual risk of HIV among Latino and Black men who have sex with men. American Journal of Public Health, 102 Suppl , S242-9. doi:10.2105/AJPH.2011.300641

Baldwin, J. A., Maxwell, C. J. C., Fenaughty, A. M., \& Trotter, R. T. (2000). Alcohol as a Risk Factor for HIV Transmission Among American Indian and Alaska Native Drug Users. American Indian and Alaska Native Mental Health Research (Online), 9(1), 1-16. 
Balsam, K. F., Huang, B., Fieland, K. C., Simoni, J. M., \& Walters, K. L. (2004). Culture, Trauma, and Wellness: A Comparison of Heterosexual and Lesbian, Gay, Bisexual, and Two-Spirit Native Americans. Cultural Diversity \& Ethnic Minority Psychology, 10(3), 287-301. doi:10.1037/1099-9809.10.3.287

Barney, D. D. (2003). Health risk-factors for gay American Indian and Alaska Native adolescent males. Journal of Homosexuality, 46(1-2), 137-57. Retrieved from http://www.ncbi.nlm.nih.gov/pubmed/15086222

Battle, J., Cohen, C. J., Warren, D., Fergerson, G., \& Audam, S. (2002). Say it Loud I'm Black and I'm Proud. New York, NY: The Policy Institute of the National Gay and Lesbian Task Force.

Baum, A., \& Polsluszny, A. (1999). Mapping biobehavioral Contributions to Health and Illness. Annual Review of Psychology, 50(1), 137-163.

Belcourt-Dittloff, A., \& Stewart, J. (2000). Historical Racism: Implications for Native Americans. American Psychologist, 55(10), 1166-1167. doi:10.1037//0003066X.55.10.1152

Bennett, G. G., Wolin, K. Y., Robinson, E. L., Fowler, S., \& Edwards, C. L. (2005). Perceived racial/ethnic harassment and tobacco use among African American young adults. American Journal of Public Health, 95(2), 238-40. doi:10.2105/AJPH.2004.037812

Benotsch, E. G., Kalichman, S. C., \& Cage, M. (2002). Men Who Have Met Sex Partners Via The Internet: Prevalence, Predictors, and Implications For HIV Prevention. Archives of Sexual Behavior, 31, 177-183.

Beresford, B. A. (1994). Self-Efficacy Mechanism in Human Agency. Journal of Child Psychology and Psychiatry and Allied Disciplines, 35, 171-209.

Bertolli, J., Lee, L. M., \& Sullivan, P. S. (2007). Racial Misidentification of American Indians/Alaska Natives in th HIV/AIDS Reporting Systems of Five States and One Urban Health Jurisdiction, U.S., 1984-2002. Public Health Reports, 122(3), 382-392.

Bertolli, J., McNaghten, a D., Campsmith, M., Lee, L. M., Leman, R., Bryan, R. T., \& Buehler, J. W. (2004). Surveillance systems monitoring HIV/AIDS and HIV risk behaviors among American Indians and Alaska Natives. AIDS Education and Prevention : Official Publication of the International Society for AIDS Education, 16(3), 218-37. doi:10.1521/aeap.16.3.218.35442 
Bingham, T., Hawara, N., Johnson, E., Secura, G., MacKellar, D., \& Valleroy, L. (2003). The Effect Of Partner Characteristics On HIV Infection Among African American Men Who Have Sex With Men In The Young Men's Survey, Los Angeles, 19902000. AIDS Education and Prevention, 15, 39-52.

Black, D., Gates, G., Sanders, S., \& Taylor, L. (2000). Demographics of the gay and lesbian populations in the United States: Evidence from the Available Systematic Data Sources. Demography, 37, 137-154.

Blais, M. (2006). Vulnerability to HIV among Regular Male Parnters and the Social Coding of Intimacy in Modern Societies. Culture, Health \& Sexuality, 8(1), 31-44.

Blum, R., Harmon, B., Harris, L., Bergeisen, L., \& Resnick, M. (1992). American Indian-Alaska Native youth health. Jounral of the American Medical Association, 267(12), 1637-1644.

Bonilla-Silva, E. (1997). Rethinking Racism: Toward a Structural Interpretation. American Sociological Review, 62(3), 465-480.

Bouey, P. D., \& Duran, B. E. S. (2000). The Ahalaya Case-Management Program for HIV-Infected American Indians, Alaska Natives, and Native Hawaiians: Quantitative and Qualitative Evaluations of Impacts. American Indian and Alaska Native Mental Health Research (Online), 9(6), 36-52.

Branscombe, N. R., Schmitt, M. T., \& Harvey, R. (1999). Perceived Pervasive Discrimination among African Americans: Implications for Group Identification and Well-being. Journal of Personality and Social Psychology, 77(1), 135-149.

Brooks, V. R. (1981). Minority Stress in Lesbian Women. Lexington, Mass: Lexington Books.

Brown, L. B. (1997). Women and Men, Not-Men and Not-Women, Lesbians and Gays: American Indian Gender Style Alternatives. Journal of Gay \& Lesbian Social Services, 6(2), 5-20.

Bruce, D., Ramirez-Valles, J., \& Campbell, R. T. (2008). Stigmatization, Substance Use, and Sexual Risk Behavior Among Latino Gay and Bisexual Men and Transgender Persons. The Journal of Drug Issues, 38(1), 235-260.

Burks, D. J., Robbins, R., \& Durtschi, J. P. (2011). American Indian gay, bisexual and two-spirit men: a rapid assessment of HIV/AIDS risk factors, barriers to prevention and culturally-sensitive intervention. Culture, Health \& Sexuality, 13(3), 283-98. doi:10.1080/13691058.2010.525666 
Butler, J. (1993). Bodies that Matter. New York, NY: Routledge.

Calabrese, S. K., Reisen, C. a, Zea, M. C., Poppen, P. J., \& Bianchi, F. T. (2012). The pleasure principle: the effect of perceived pleasure loss associated with condoms on unprotected anal intercourse among immigrant Latino men who have sex with men. AIDS Patient Care and STDs, 26(7), 430-5. doi:10.1089/apc.2011.0428

Caldwell, J. C. (2000). Rethinking the African AIDS Epidemic. Population and Development Review, 26(1), 117-135.

Cassels, S., Pearson, C. R., Walters, K., Simoni, J. M., \& Morris, M. (2011). Sexual Partner Concurrency and Sexual Risk among Gay, Lesbian, Bisexual, and Transgender American Indian/Alaska Natives. Sexually Transmitted Diseases, 37(4), 272-278. Retrieved from http://www.ncbi.nlm.nih.gov/pmc/articles/PMC3118268/

Centers for Disease Control and Prevention. (2003a). AIDS Cases by Age Group, Exposure Category, and Sex, Reported Through December 2001, United States.

Centers for Disease Control and Prevention. (2003b). Cases of HIV Infection and AIDS in the United States, by race/ethnicity, 1998-2002. Atlanta, GA.

Centers for Disease Control and Prevention. (2004). HIV Transmission AMong Black College Student and Non-College Men Who Have Sex With Men - North Carolina, 2003. MMWR Morbidity and Mortality Weekly Report, 53(52), 731-734.

Centers for Disease Control and Prevention. (2009). Sexual and Reproductive Health of Persons Aged 10-24 Years - United States, 2002-2007. Surveillance Summaries, MMWR, 58.

Centers for Disease Control and Prevention. (2010). HIV in the United States (Vol. 57, pp. 1-2).

Centers for Disease Control and Prevention. (2013). HIV Surveillance Report, 2011. Atlanta, GA.

Chae, D. H., Krieger, N., Bennett, G. G., Lindsey, J. C., Stoddard, A. M., \& Barbeau, E. M. (2010). Implications of Discrimination Based on Sexuality, Gender, and Race/Ethnicity for Psychological Distress among Working-Class Sexual Minorities: The United for Health Study, 2003-2004. International Journal of Health Services, 40(4), 589-608. doi:10.2190/HS.40.4.b 
Chae, D. H., \& Walters, K. L. (2009). Racial discrimination and racial identity attitudes in relation to self-rated health and physical pain and impairment among two-spirit American Indians/Alaska Natives. American Journal of Public Health, 99 Suppl 1, S144-51. doi:10.2105/AJPH.2007.126003

Chae, D. H., \& Yoshikawa, H. (2008). Perceived group devaluation, depression, and HIV-risk behavior among Asian gay men. Health Psychology : Official Journal of the Division of Health Psychology, American Psychological Association, 27(2), 140-8. doi:10.1037/0278-6133.27.2.140

Chen, Y., \& Tryon, G. S. (2012). Dual Minority Stress and Asian American Gay Men's Psychological Distress. Journal of Community ..., 40(5), 539-554. doi:10.1002/jcop

Chng, C. L., \& Geliga-Vargas, J. (2000). Ethnic identity, gay identity, sexual sensation seeking and HIV risk taking among multiethnic men who have sex with men. AIDS Education and Prevention: Official Publication of the International Society for AIDS Education, 12(4), 326-339.

Choi, K.-H., Lew, S., Vittinghoff, E., Catania, J., Barrett, D., \& Coates, T. (1996). The efficacy of brief group counseling in HIV risk reduction among homosexual Asian and Pacific Islander men. AIDS, 10(1), 81-87.

Choi, K.-H., Operario, D., Gregorich, S. E., McFarland, W., MacKellar, D., \& Valleroy, L. (2005). Substance Use, Substance Choice, and Unprotected Anal Intercourse Among Young Asian American and Pacifica Islander Men Who Have Sex With Men. AIDS Education and Prevention, 17(5), 418-429.

Choi, K.-H., Paul, J., Ayala, G., Boylan, R., \& Gregorich, S. E. (2013). Experiences of Discrimination and Their Impact on the Mental Health among Africian American, Asian and Pacific Islander, and Latino Men Who Have Sex with Men. American Journal of Public Health, 103(5), 868-874. doi:10.2105/AJPH.2012.301052.Experiences

Choo, H. Y., \& Ferree, M. M. (2010). Practicing Intersectionality in Sociological Research: A Critical Analysis of Inclusions, Interactions, and Institutions in the Study of Inequalities. Sociological Theory, 28(2), 129-149.

Cockerham, W. C. (2005). Health Lifestyle Theory and the Convergence of Agency and Structure. Journal of Health and Social Behavior, 46(1), 51-67. doi:10.1177/002214650504600105 
Colfax, G., Vittinghoff, E., Husnik, M. J., McKirnan, D., Buchbinder, S., Koblin, B. A., ... EXPLORE Study Team. (2004). Substance Use and Sexual Risk: A Participantand Episode-level Analysis among a Cohort of Men Who Have Sex with Men. American Journal of Epidemiology, 159(10), 1002-1012.

doi:10.1093/aje/kwh135

Collins, P. H. (1990). Black Feminist Thought: Knowledge, Consciousness, and the Politics of Empowerment. Boston: Unwin Hyman.

Conway, G. A., Ambrose, T. J., Chase, E., Hooper, E. Y., Helgerson, S. D., Johannes, P., ... Dondero, T. J. (1992). HIV Infection in American Indians and Alaska Natives: Surveys in the Indian Health Service. Journal of Acquired Immune Deficiency Syndrome, 5, 803-809.

Crawford, I., Allison, K. W., Zamboni, B. D., \& Soto, T. (2002). The influence of dualidentity development on the psychosocial functioning of African-American gay and bisexual men. Journal of Sex Research, 39(3), 179-89.

doi:10.1080/00224490209552140

Crenshaw, K. (1991). Mapping the Margins: Intersectionality, Identity Politics, and Violence against Women of Color. Standford Law Review, 43, 1241-1299.

Crepaz, N., Hart, T., \& Marks, G. (2004). Highly Active Antiretroviral Therapy Are Associated With Sexual Risk Taking Among HIV-Infected And Uninfected Homosexual Men. AIDS, 16, 775-780.

Crepaz, N., \& Marks, G. (2001). Are Negative Affective States Associated With HIV Sexual Risk Behaviors? A Meta-Analytic Review. Health Psychology, 20(4), 291299. Retrieved from http://psycnet.apa.org/journals/hea/20/4/291/

Crepaz, N., \& Marks, G. (2002). Towards an understanding of sexual risk behavior in people living with HIV: a review of social, psychological, and medical findings. AIDS (London, England), 16(2), 135-49. Retrieved from http://www.ncbi.nlm.nih.gov/pubmed/11807297

Crepaz, N., Marks, G., Mansergh, G., Murphy, S., Miller, C., \& Appleby, P. R. (2000). Age-Related Risk For HIV Infection In Men Who Have Sex With Men: Examination Of Behavioral, Relationship, And Serostatus Variables. AIDS Education and Prevention, 12(5), 405-415.

Davidovich, U., de Wit, J., Albrecht, N., Geskus, R., Stroebe, W., \& Coutinho, R. (2001). A 17-Year Study Of Seroconversion Among Gay Men. AIDS, 15(10), 1303-1308. 
Davidovich, U., de Wit, J., \& Stroebe, W. (2004). Behavioral and COgnitive Barriers to Safer Sex between Men in Steadt Relationships: Implication for Prevention Strategies. AIDS Education and Prevention, 16(4), 304-314.

Davidovich, U., de Wit, J., \& Stroebe, W. (2006). Relationship Charateristics and Risk of HIV Infection: Rusbult's Investment Model and Sexual Risk Behavior of Gay Men in Steady Relationships. Journal of Applied Social Psychology, 36(1), 22-40.

De Beauvoir, S. (1953). The Second Sex. New York, NY: Knopf.

De Santis, J. P., Colin, J. M., Provencio Vasquez, E., \& McCain, G. C. (2008). The relationship of depressive symptoms, self-esteem, and sexual behaviors in a predominantly Hispanic sample of men who have sex with men. American Journal of Men's Health, 2(4), 314-21. doi:10.1177/1557988307312883

De Vroome, E. M. M., Stroebe, W., Sandfort, T. G. M., de Wit, J., \& Van Griensven, G. J. P. (2000). Safer Sex in Social Context: Individualistic and Relational Determinants of AIDS-Preventive Behavior Among Gay Men. Journal of Applied Social Psychology, 30(11), 2322-40.

Dean, H. D., \& Fenton, K. A. (2010). Addressing social determinants of health in the prevention and control of HIV/AIDS, viral hepatitis, sexually transmitted infections, and tuberculosis. Public Health Reports, 125(Suppl 4), 1-5.

Diamond, C., Davidson, A., Sorvillo, F., \& Buskin, S. (2001). HIV-infected American Indians/Alaska Natives in the Western United States. Ethnicity and Disease, 11(4), 633-644.

Diaz, R. M., \& Ayala, G. (2001). Social Discrimination and Health: The Case of Latino Gay Men and HIV Risk. New York, NY: Policy Institute of the National Gay and Lesbian Task Force.

Diaz, R. M., Ayala, G., \& Bein, E. (2004). Sexual Risk as an Outcome of Social Oppression: Data from a Probability Sample of Latino Gay men in Three U.S. Cities. Cultural Diversity and Ethnic Minority Psychology, 10(3), 255-267.

Díaz, R. M., Ayala, G., Bein, E., Henne, J., \& Marin, B. V. (2001). The impact of homophobia, poverty, and racism on the mental health of gay and bisexual Latino men: findings from 3 US cities. American Journal of Public Health, 91(6), 927-32. Retrieved from http://www.pubmedcentral.nih.gov/articlerender.fcgi?artid=1446470\&tool=p mcentrez\&rendertype=abstract 
Dill, B. T., \& Zambrana, R. E. (2009). Emerging intersections: Race, class and gender in theory, policy and practice. New Brunswick, NJ: Rutgers University Press.

Dovidio, J. F., \& Gaertner, S. L. (2000). Aversive Racism and Selective Decisions: 1989-1999. Psychological Science, 11, 315-319.

Duran, B., Bulterys, M., Iralu, J., Graham, C., Edwards, A., \& Harrison, M. (2000). American Indians with HIV/AIDS: Health and Social Service Needs, Barriers to Care, and Satisfaction with Services among a Western Tribe. American Indian and Alaska Native Mental Health Research (Online), 9(2), 22-35.

Duran, E., \& Duran, B. (1995). Native American Postcolonial Psychology. Albany, NY: State University of New York Press.

Duran, E., Duran, B., Yellow Horse Brave Heart, M., \& Yellow Horse-Davis, S. (1998). Healing the American Indian Soul Wound. In Y. Danieli (Ed.), International Handbook of Multigenerational Legacies of Trauma (pp. 341-354). New York, NY: Plenum.

Duran, E., Firehammer, J., \& Gonzalez, J. (2008). Liberation Psychology as the Path Toward Healing Cultural Soul Wounds. Journal of Counseling \& Development, 86(3), 288-295. doi:10.1002/j.1556-6678.2008.tb00511.x

Ekstrand, M. L., Stall, R. D., Paul, J. P., Osmond, D. H., \& Coates, T. J. (1999). Gay Men Report High Rates Of Unprotected Anal Sex With Partners Of Unknown Or Discordant HIV Status. AIDS, 13(12), 1525-1533.

Elford, J., Bolding, G., Maguire, M., \& Sherr, L. (1999). Sexual Risk Behaviour Among Gay Men In A Relationship. AIDS, 13(11), 1407-1411.

Elford, J., \& Hart, G. (2003). If HIV Prevention Works, Why Are Rates of High-Risk Sexual Behavior Increasing Among MSM. AIDS Education and Prevention, 15(4), 294-308.

Farmer, P. (1999). Infections and Inequalities: The Modern Plagues. Berkeley, CA: University of California Press.

Farmer, P. (2005). Pathologies of Power: Health, Human Rights, and The New War on the Poor. Berkeley, CA: University of California Press.

Frijida, N. H. (1988). The Laws of Emotion. American Psychologist, 43, 349-358. 
Gibbons, F. X., Gerrard, M., Cleveland, M. J., Wills, T. a, \& Brody, G. (2004). Perceived discrimination and substance use in African American parents and their children: a panel study. Journal of Personality and Social Psychology, 86(4), 51729. doi:10.1037/0022-3514.86.4.517

Goffman, E. (1963). Stigma: Notes on the Management of Spoiled Identity. New York, NY: Simon \& Schuster.

Gone, J. P. (2008). Mental Health Discourse as Western Cultural Proselytization. Ethos, 36, 310-315.

Halkitis, P. N., Zade, D. D., Shrem, M., \& Marmor, M. (2004). Beliefs About HIV NonInfection And Risky Sexual Behavior Among MSM. AIDS Education and Prevention, 16, 448-458.

Hall, H. I., Byers, R. H., Ling, Q., \& Espinoza, L. (2007). Racial/ethnic and age disparities in HIV prevalence and disease progression among men who have sex with men in the United States. American Journal of Public Health, 97(6), 1060-6. doi:10.2105/AJPH.2006.087551

Hall, R. L., Wilder, D., Bodenroeder, P., \& Hess, M. (1990). Assessment of AIDS knowledge, attitudes, behaviors, and risk level of Northwestern American Indians. American Journal of Public Health, 80(7), 875-7. Retrieved from http://www.pubmedcentral.nih.gov/articlerender.fcgi?artid=1404982\&tool=p mcentrez\&rendertype=abstract

Halpern, C. T., Hallfors, D., Bauer, D. J., Iritani, B., Waller, M. W., \& Cho, H. (2004). Implications of racial and gender differences in patterns of adolescent risk behavior for HIV and other sexually transmitted diseases. Perspectives on Sexual and Reproductive Health, 36(6), 239-47. doi:10.1363/psrh.36.239.04

Han, C. (2007). They Don't Want To Cruise Your Type: Gay Men of Color and the Racial Politics of Exclusion. Social Identities, 13(1), 51-67. doi:10.1080/13504630601163379

Han, C. (2008). A qualitative exploration of the relationship between racism and unsafe sex among Asian Pacific Islander gay men. Archives of Sexual Behavior, 37(5), 827-37. doi:10.1007/s10508-007-9308-7

Hart, T., \& Peterson, J. L. (2004). Predictors of risky sexual behavior among young African American men who have sex with men. American Journal of Public Health, 94(7), 1122-4. Retrieved from http://www.pubmedcentral.nih.gov/articlerender.fcgi?artid=1448408\&tool=p mcentrez\&rendertype=abstract 
Health Canada. (1998). Taking Action on Population Health: A Position Paper for Health Promotion and Programs Branch Staff. Ottawa.

Heffernan, C. (2002). HIV, Sexually Transmitted Infections and Social Inequalities: When the Transmission is more Social than Sexual. International Journal of Sociology and Social Policy, 22(4/5/6), 159-176. Retrieved from http://www.emeraldinsight.com/journals.htm?articleid=850419\&show=abstr act

Hequembourg, A. L., \& Brallier, S. a. (2009). An exploration of sexual minority stress across the lines of gender and sexual identity. Journal of Homosexuality, 56(3), 273-98. doi:10.1080/00918360902728517

Herek, G. M. (1990). The Context of Anti-Gay Violence: Notes on Cultural and Psychological Heterosexism. Journal of Interpersonal Violence, 5(3), 316-333. doi:10.1177/088626090005003006

Herek, G. M., Gillis, J., \& Cogan, J. (1999). Psychological sequelae of hate-crime victimization among lesbian, gay, and bisexual adults. The Journal of Consulting and Clinical Psychology, 67, 945-951.

Hirshfield, S., Remien, R. H., Humberstone, M., Walavalkar, I., \& Chiasson, M. a. (2004). Substance use and high-risk sex among men who have sex with men: a national online study in the USA. AIDS Care, 16(8), 1036-47.

doi:10.1080/09540120412331292525

Hoff, C. C., Chakravarty, D., Beougher, S. C., Barbes, L., Dadasovich, R., \& Neilands, T. (2009). Serostatus Differences And Agreements About Sex With Outside Partners Among Gay Male Couples. AIDS Education and Prevention, 25(1), 2538.

House, J. S., \& Williams, D. R. (2003). Understanding and Reducing Socioeconomic and Racial Disparities in Health. In R. Hofrichter (Ed.), Health and Social Justice: Politics, Ideology, ad Inequity in the Distribution of Disease - A Public Health Reader (pp. 89-131). San Francisco, CA: Jossey-Bass.

Jacobs, S.-E., Thomas, W., \& Lang, S. (1997). Two-spirit people: Native American gender identity, sexuality, and spirituality. (S.-E. Jacobs, W. Thomas, \& S. Lang, Eds.). Chicago, IL: Univerisity of Illinois Press.

Janssen, M., De Wit, J., Stroebe, W., \& Griensven, F. (2000). Educational Status and Risk of HIV in Young Gay Men. Journal of Health Psychology, 5(4), 487-99. doi:10.1177/135910530000500407 
Jarama, S. L., Kennamer, J. D., Poppen, P. J., Hendricks, M., \& Bradford, J. (2005). Psychosocial, behavioral, and cultural predictors of sexual risk for HIV infection among Latino men who have sex with men. AIDS and Behavior, 9(4), 513-23. doi:10.1007/s10461-005-9022-1

Jary, D., \& Jary, J. (1995). Collins Dictionary of Sociology. (D. Jary \& J. Jary, Eds.) (2nd ed.). Glasgow, UK: Haper Collins Publishers.

Jin, F., Prestage, G., Ellard, J., Kippax, S., Kaldor, J. M., \& Grulich, A. E. (2007). How Homosexual Men Believe They Became Infected With HIV: The Role of RiskReduction Behaviors. Journal of Acquired Immune Deficiency Syndrome, 46, 245247.

Kalichman, S. C. (2000). HIV Transmission Risk Behaviors Of Men And Women Living With HIV-AIDS: Prevalence, Predictors, and Emerging Clinical Interventions. Clinical Psychology, 7, 32-47.

Kalichman, S. C., Tannenbaum, L., \& Nachimson, D. (1998). Personality and Cognitive Factors Influencing Substance Use and Sexual Risk for HIV Infection Among Gay and Bisexual Men. Psychology of Addictive Behaviors: Journal of the Society of Psychologists in Addictive Behaviors, 12(4), 262-271.

Karon, J. M., Fleming, P. L., Steketee, R. W., \& De Cock, K. M. (2001). HIV in the United States at the turn of the century: an epidemic in transition. American Journal of Public Health, 91(7), 1060-8. Retrieved from http://www.pubmedcentral.nih.gov/articlerender.fcgi?artid=1446722\&tool=p mcentrez\&rendertype=abstract

Kaufman, C. E., Shelby, L., Mosure, D. J., Marrazzo, J., Wong, D., de Ravello, L., ... Cheek, J. E. (2007). Within the hidden epidemic: sexually transmitted diseases and HIV/AIDS among American Indians and Alaska Natives. Sexually Transmitted Diseases, 34(10), 767-77. doi:10.1097/01.olq.0000260915.64098.cb

Kelly, J. A., Hoffman, R. G., Rompa, D., \& Gray, M. (1998). Protease Inhibitor Combination Therapies And Perceptions Of Gay Men Regarding AIDS Severity And The Need To Maintain Safer Sex. AIDS, 12, F91-F95.

Kippax, S., Noble, J., Prestage, G., Crawford, J. M., Campbell, D., Baxter, D., \& et al. (1997). Sexual Negotiation In The AIDS Era: Negotiated Safety Revisited. AIDS, 11, 191-197. 
Koblin, B. A., Husnik, M. J., Colfax, G., Huang, Y., Madison, M., Mayer, K., ... Buchbinder, S. (2006). Risk Factors for HIV Infection Among Men Who Have Sex With Men. AIDS, 20, 731-739.

Koblin, B. A., Perdue, T., \& Ren, L. (2003). Attitudes About COmbination HIV Therapies: The Next Generation Of Gay Men At Risk. Journal of Urban Health, 80, 510-519.

Koopman, J. S. (1996). Emerging Objectives and Methods in Epidemiology. American Journal of Public Health, 86(5), 630-632.

Krieger, N. (2000). Discrimination and Health. In L. F. Berkman \& I. Kawachi (Eds.), Social Epidemiology (pp. 36-75). New York, NY: Oxford University Press.

Krieger, N. (2001). A Glossary for Social Epidemiology. Journal of Epidemiology and Community Health, 55(10), 693-700.

Krieger, N. (2003). Does racism harm health? Did child abuse exist before 1962? On explicit questions, critical science, and current controversies: an ecosocial perspective. American Journal of Public Health, 98(9 Suppl), S20-5. Retrieved from

http://www.pubmedcentral.nih.gov/articlerender.fcgi?artid=2518569\&tool=p mcentrez\&rendertype=abstract

Lane, S. D., Rubinstein, R. a, Keefe, R. H., Webster, N., Cibula, D. a, Rosenthal, A., \& Dowdell, J. (2004). Structural violence and racial disparity in HIV transmission. Journal of Health Care for the Poor and Underserved, 15(3), 319-35. Retrieved from http://www.ncbi.nlm.nih.gov/pubmed/15453172

Lapidus, J. a, Bertolli, J., McGowan, K., \& Sullivan, P. (2006). HIV-related risk behaviors, perceptions of risk, HIV testing, and exposure to prevention messages and methods among urban American Indians and Alaska Natives. AIDS Education and Prevention : Official Publication of the International Society for AIDS Education, 18(6), 546-59. doi:10.1521/aeap.2006.18.6.546

LaVeist, T. A. (2000). On the study of race, racism, and health: a shift from description to explanation. International Journal of Health Services : Planning, Administration, Evaluation, 30(1), 217-9. Retrieved from http://www.ncbi.nlm.nih.gov/pubmed/10707307

Lazarus, R. S., \& Folkman, S. (1984). Coping and Adaptation. In W. D. Gentry (Ed.), Handbook of Behavioral Medicine. New York, NY: The Guilford Press. 
Lehavot, K., Walters, K. L., \& Simoni, J. M. (2009). Abuse, mastery, and health among lesbian, bisexual, and two-spirit American Indian and Alaska Native women. Cultural Diversity \& Ethnic Minority Psychology, 15(3), 275-84. doi:10.1037/a0013458

Lehrer, J. A., Shrier, L. A., Gortmaker, S., \& Buka, S. (2006). Depressive Symptoms as a Longitudinal Predictor of Sexual Risk Behaviors Among US Middle and High School Students. Pediatrics, 118(1), 189-200.

Liau, A., Millett, G. A., \& Marks, G. (2006). Meta-Anlytic Examination of Online SexSeeking And Sexual Risk Behavior Among Men Who Have Sex With Men. Sexually Transmitted Diseases, 33, 576-584.

Mansergh, G., Colfax, G. N., Marks, G., Rader, M., Guzman, R., \& Buchbinder, S. (2001). The Circuit Party Men's Health Survey: Findings And Implications For Gay And Bisexual Men. American Journal of Public Health, 91, 953-953.

Mansergh, G., \& Marks, G. (1998). Age and risk of HIV infection in men who have sex with men. AIDS (London, England), 12(10), 1119-28. Retrieved from http://www.ncbi.nlm.nih.gov/pubmed/11063060

Marks, G., Bingham, C. R., \& Duval, T. S. (1998). Negative Affect and Unsafe Sex in HIV-Positive Men. AIDS and Behavior, 2(2), 89-99.

Marks, G., Crepaz, N., Senterfitt, J. W., \& Janssen, R. S. (2005). Meta-Analysis of HighRisk Sexual Behavior in Persons Aware and Unaware They Are Infected With HIV In The United States: Implications For HIV Prevention Programs. Journal of Acquired Immune Deficiency Syndrome, 39, 446-453.

Marks, G., Millett, G. a, Bingham, T., Lauby, J., Murrill, C. S., \& Stueve, A. (2010). Prevalence and protective value of serosorting and strategic positioning among Black and Latino men who have sex with men. Sexually Transmitted Diseases, 37(5), 325-7. doi:10.1097/OLQ.0b013e3181c95dac

Marmot, M. G., \& Wilkinson, R. G. (1999). Social Determinants of Health. Oxford: Oxford University Press.

Martin, J. K., Tuch, S. a, \& Roman, P. M. (2003). Problem drinking patterns among African Americans: the impacts of reports of discrimination, perceptions of prejudice, and "risky" coping strategies. Journal of Health and Social Behavior, 44(3), 408-25. Retrieved from

http://www.ncbi.nlm.nih.gov/pubmed/14582316 
Martinot, S. (2003). The Rule of Racialization: Class, Identity, and Governance. Philadelphia: Temple University Press.

Mays, V. M., \& Cochran, S. D. (2001). Mental health correlates of perceived discrimination among lesbian, gay, and bisexual adults in the United States. American Journal of Public Health, 91(11), 1869-76. Retrieved from http://www.pubmedcentral.nih.gov/articlerender.fcgi?artid=1446893\&tool=p mcentrez\&rendertype=abstract

McCall, L. (2005). The Complexity of Intersectionality. Signs, 30(3), 1771-1800.

McKirnan, D., Ostrow, D., \& Hope, B. (1996). Sex, drugs and escape: a psychological model of HIV-risk sexual behaviours. AIDS Care, 8(6), 655-669.

McLean, J., Boulton, M., \& Brookes, M. (1994). Regular Partners and Risky Behaviour: Why Do Gay Men Have Unprotected Intercourse? AIDS Care, 6, 331341.

McNaghten, a D., Neal, J. J., Li, J., \& Fleming, P. L. (2005). Epidemiologic profile of HIV and AIDS among American Indians/Alaska Natives in the USA through 2000. Ethnicity \& Health, 10(1), 57-71. doi:10.1080/1355785052000323038

McNeal, J. L. (1997). The Association of Idealization and Intimacy Factors with Condom Use in Gay Male Couples. Journal of Clinical Psychology in Medical Settings, 4(4), 437-451.

Mead, L. T. T. R. (1996). Nga Aho O Te Kakahu Matauranga: The Multiple Layers of Struggle by Maori in Education. University of Auckland.

Meyer, I. H. (1995). Minority Stress and Mental Health in Gay Men. Journal of Health and Social Behavior, 36(1), 38-56.

Meyer, I. H. (2003). Prejudice, social stress, and mental health in lesbian, gay, and bisexual populations: conceptual issues and research evidence. Psychological Bulletin, 129(5), 674-97. doi:10.1037/0033-2909.129.5.674

Meyer, I. H. (2010). Identity, Stress, and Resilience in Lesbians, Gay Men, and Bisexuals of Color. The Counseling Psychologist, 38(3), 442-454. doi:10.1177/0011000009351601

Millett, G. A., Peterson, J. L., Wolitski, R. J., \& Stall, R. (2006). Greater Risk for HIV Infection of Black Men Who Have Sex With Men: A Critical Literature Review. American Journal of Public Health, 96(6), 1007-1020. doi:10.2105/AJPH 
Mills, T. C., Stall, R. D., Pollack, L., Paul, J. P., Binson, D., Canchola, J., \& Catania, J. A. (2001). Health-related Characteristics of Men Who Have Sex With Men: A Comparison of Those Living in "Gay Ghettos" with Those Living Elsewhere. American Journal of Public Health, 91, 980-983.

Mitchell, J. W. (2013). HIV-Negative and HIV-Discordant Gay Male Couples' Use of HIV Risk-Reduction Strategies: Differences by Partner Type and Couples' HIVStatus. AIDS and Behavior, 17, 1557-1569.

Mitchell, J. W. (2014). Characteristics and Allowed Behaviors of Gay Male Couples' Sexual Agreements. Journal of Sex Research, 51(3), 316-328.

Mizuno, Y., Borkowf, C., Millett, G. a, Bingham, T., Ayala, G., \& Stueve, A. (2012). Homophobia and racism experienced by Latino men who have sex with men in the United States: correlates of exposure and associations with HIV risk behaviors. AIDS and Behavior, 16(3), 724-35. doi:10.1007/s10461-011-9967-1

Mojola, S. a, \& Everett, B. (2012). STD and HIV risk factors among U.S. young adults: variations by gender, race, ethnicity and sexual orientation. Perspectives on Sexual and Reproductive Health, 44(2), 125-33. doi:10.1363/4412512

Moreau-Gruet, F., Jeannin, A., Dubois-Arber, F., \& Spencer, B. (2001). Management Of The Risk Of HIV Infection In Male Homosexual Couples. AIDS, 15(8), 10251035.

Myers, H. F., Javanbakht, M., Martinez, M., \& Obediah, S. (2003). Psychosocial predictors of risky sexual behaviors in African American men: implications for prevention. AIDS Education and Prevention : Official Publication of the International Society for AIDS Education, 15(1 Suppl A), 66-79. Retrieved from http://www.ncbi.nlm.nih.gov/pubmed/12630600

Nakamura, N., \& Zea, M. C. (2010). Experiencesof Homonegativity and Sexual Risk Behaviour in a Sample of Latino Gay and Bisexual Men. Culture, Health \& Sexuality, 12(1), 73-85.

Nemoto, T., Iwamoto, M., \& Operario, D. (2003). HIV risk behaviors among Asian and Pacific Islander male-to-female transgenders. The Community Psychologist, 36, 31-35.

Nemoto, T., Sausa, L. A., Operario, D., \& Keatley, J. (2006). Need for HIV/AIDS education and intervention for MTF transgenders: Responding to the challenge. Journal of Homosexuality, 51(1), 183-202. 
Noh, S., \& Kaspar, V. (2003). Perceived discrimination and depression: moderating effects of coping, acculturation, and ethnic support. American Journal of Public Health, 93(2), 232-8. Retrieved from

http://www.pubmedcentral.nih.gov/articlerender.fcgi?artid=1447722\&tool=p mcentrez\&rendertype=abstract

O'Neil, J. D. (1986). The Politics of Health in the Fourth World: A Northern Canadian Example. Human Organization, 45, 119-128.

Omi, M., \& Winant, H. (1986). Racial Formation in the United States: From the $1960 \mathrm{~s}$ to the 1980s. New York, NY: Russel Sage Foundation.

Parker, R. (2001). Sexuality, Culture, and Power in HIV/AIDS Research. Annual Review of Anthropology, 30, 163-179.

Parker, R., \& Aggleton, P. (2003). HIV and AIDS-related stigma and discrimination: a conceptual framework and implications for action. Social Science \& Medicine (1982), 57(1), 13-24. Retrieved from http://www.ncbi.nlm.nih.gov/pubmed/12753813

Parsons, J., \& Kutnick, A. (2005). Sexual risk behaviors and substance use among alcohol abusing HIV-positive men who have sex with men. ... of Psychoactive Drugs, 37(1), 27-36. Retrieved from http://www.tandfonline.com/doi/full/10.1080/02791072.2005.10399746

Parsons, J. T., Schrimshaw, E. W., Wolitski, R. J., Halkitis, P. N., Purcell, D. W., Hoff, C. C., \& et al. (2005). Sexual Harm Reduction Preactices Of HIV Seropositive Gay And Bisexual Men: Serosorting, Strategic Positioning, And Withdrawl Before Ejaculation. AIDS, 19(Suppl 1), S13-S25.

Pearson, C. R., Walters, K. L., Simoni, J. M., Beltran, R., \& Nelson, K. M. (2013). A Cautionary Tale: Risk Reduction Strategies Among Urban American Indian/Alaska Native Men Who Have Sex With Men. AIDS Education and Prevention : Official Publication of the International Society for AIDS Education, 25(1), 25-37. doi:10.1521/aeap.2013.25.1.25

Perdue, T., Hagan, H., Thiede, H., \& Valleroy, L. (2003). Depression and HIV Risk Behavior Among Seattle-Area Injection Drug Users and Young Men Who Have Sex With Men. AIDS Education and Prevention, 15(1), 81-92.

Peterson, J. L., Bakeman, R., \& Stall, R. D. (2001). The Community Intervention Trail For Youth Study Team: Racial/Ethinc Patterns Of HIV Sexual Risk Behaviors Among Young Men Who Have Sex With Men. Journal of the Gay and Lesbian Medical Association, 5, 1007-1019. 
Peterson, J. L., Coates, T. J., Catania, J. A., Middleton, L., Hilliard, B., \& Hearst, N. (1992). High-Risk Sexual Beahvior And Condom Use Among Gay and Bisexual African-American Men. American Journal of Public Health, 82, 1490-1494.

Peterson, J. L., Folkman, S., \& Bakeman, R. (1996). Stress, Coping, HIV Status, Psychological Resources, and Depressive Mood in African American Gay, Bisexual, and Heterosexual Men. American Journal of Community Psychology, 24, 461-487.

Phillips, A. F., \& Pirkle, C. M. (2011). Moving beyond behaviour: advancing HIV risk prevention epistemologies and interventions (A report on the state of the literature). Global Public Health, 6(6), 577-592. Retrieved from http://www.tandfonline.com/doi/abs/10.1080/17441692.2011.584325

Poppen, P. J., Reisen, C. a, Zea, M. C., Bianchi, F. T., \& Echeverry, J. J. (2004). Predictors of unprotected anal intercourse among HIV-positive Latino gay and bisexual men. AIDS and Behavior, 8(4), 379-89. doi:10.1007/s10461-004-73225

Poundstone, K. E., Strathdee, S. a, \& Celentano, D. D. (2004). The social epidemiology of human immunodeficiency virus/acquired immunodeficiency syndrome. Epidemiologic Reviews, 26, 22-35. doi:10.1093/epirev/mxh005

Prestage, G., Jin, F., Grulich, A., de Wit, J., \& Zablotska, I. B. (2011). Gay Men are Less Likely to Use Condoms with Casual Sex Partners They Know "Well." AIDS and Behavior, 16(3), 664-668.

Purcell, D. W., Moss, S., Remien, R. H., Woods, W. J., \& Parsons, J. T. (2006). Illicit Substance Use, Sexual Risk, HIV-Positive Gay and Bisexual Men: Differences by serostatus of Casual Partners. AIDS, 19 (nSupplement 1), S37-S47.

Radloff, L. S. (1977). The CES-D Scale: A Self Report Depression Scale for Research in the General Population. Applied Psychological Measurements, 1, 385-401.

Ramírez, H. R. (2003). “ That's My Place!”: Negotiating Racial, Sexual, and Gender Politics in San Francisco's Gay Latino Alliance, 1975-1983. Journal of the History of Sexuality, 12(2), 224-258. Retrieved from http://muse.jhu.edu/journals/sex/summary/v012/12.2roque_ramirez01.html

Ratti, R., Bakeman, R., \& Peterson, J. L. (2000). Correlates of high-risk sexual behaviour among Canadian men of South Asian and European origin who have sex with men. AIDS Care, 12(2), 193-202. 
Raymond, H. F., Chen, Y.-H., Stall, R. D., \& McFarland, W. (2011). Adolescent experiences of discrimination, harassment, connectedness to community and comfort with sexual orientation reported by adult men who have sex with men as a predictor of adult HIV status. AIDS and Behavior, 15(3), 550-6. doi:10.1007/s10461-009-9634-y

Reisner, S. L., Mimiaga, M. J., Bland, S., Skeer, M., Cranston, K., Isenberg, D., ... Mayer, K. H. (2010). Problematic alcohol use and HIV risk among Black men who have sex with men in Massachusetts. AIDS Care, 22(5), 577-87. doi:10.1080/09540120903311482

Reisner, S. L., Mimiaga, M. J., Skeer, M., Bright, D., Cranston, K., Isenberg, D., ... Mayer, K. H. (2009). Clinically significant depressive symptoms as a risk factor for HIV infection among black MSM in Massachusetts. AIDS and Behavior, 13(4), 798810. doi:10.1007/s10461-009-9571-9

Remien, R. H., Wagner, G., Carballo-Dieguez, A., \& Dolezal, C. (1998). Who May Be Engaging In High-Risk Sex Due To Medical Treatment Advances? AIDS, 12, 1560-1561.

Rogers, G., Curry, M., Oddy, J., Pratt, N., Beilby, J., \& Wilkinson, D. (2003). Depressive disorders and unprotected casual anal sex among Australian homosexually active men in primary care. HIV Medicine, 4(3), 271-5. Retrieved from http://www.ncbi.nlm.nih.gov/pubmed/12859327

Romero, A. J., \& Roberts, R. E. (2003). The Impact of Multiple Dimensions of Ethnic Identity on Discrimination and Adolescents; Self-Esteem. Journal of Applied Social Psychology, 33(11), 2288-2305.

Rouwenhorst, E., Mallitt, K.-A., \& Prestage, G. (2012). Gay Men's Use of Condoms With Casual Partners Depends on the Extent of Their Prior Acquaintance. AIDS and Behavior, 16, 1589-1596.

Rowe, M. P. (1990). Barriers to Equality: The Power of Subtle Discrimination to Maintain Unequal Opportunity. Employee Responsibilities and Rights Journal, 3, 153-163.

Rubin, D. B. (1987). Multiple Imputation for Nonresponse in Surveys. New York, NY: John Wiley and Sons.

Ruiz, J., Facer, M., \& Sun, R. (1998). Risk Factors For Human Immunodeficiency Virus Infection And Unprotected Anal Intercourse Among Younf Men Who Have Sex With Men. Sexually Transmitted Diseases, 25, 100-107. 
Samuel, M., Hessol, N., Shiboski, S., Engel, R., Speed, T., \& Winkelstein, W. J. (1993). Factors Associated with Human Immunodeficiency Virus Conversion in Homosexual Men in Three San Francisco Cohort Studies, 1984-1989. Journal of Acquired Immune Deficiency Syndrome, 6, 303-312.

Schafer, J. L. (1997). Analysis of Incomplete Multivariate Data. New York, NY: Chapman and Hall.

Schafer, J. L., \& Graham, J. W. (2002). Missing Data: Our View of the State of the Art. Psychological Methods, 7, 147-177.

Schnittker, J., \& McLeod, J. D. (2005). The Social Psychology of Health Disparities. Annual Review of Sociology, 31(1), 75-103. doi:10.1146/annurev.soc.30.012703.110622

Schwartz, S., \& Meyer, I. H. (2010). Mental health disparities research: the impact of within and between group analyses on tests of social stress hypotheses. Social Science \& Medicine (1982), 70(8), 1111-8.

doi:10.1016/j.socscimed.2009.11.032

Seage, G., Mayer, K., Horsburgh, C. J., Holmberg, S., Moon, M., \& Lamb, G. (1992). The Relation Between Nitrite Inhalants, Unprotected Receptive Anal Intercourse, And The Risk of Human Immunodeficiency Virus Infection. American Journal of Epidemiology, 135, 1-11.

Seibt, A., Ross, M., Freeman, A., Krepcho, M., Hedrich, A., McAlister, A., \& FernandezEsquer, M. (1995). Relationship between safe sex and acculturation into the gay subculture. AIDS Care, 7(Suppl 1), S85-S88.

Sellers, R. M., Caldwell, C. H., Schmeelk-Cone, K. H., \& Zimmerman, M. A. (2003). Racial Identity, Racial Discrimination, Perceived Stress, and Psychological Distress among African American Young Adults. Journal of Health and Social Behavior, 44(3), 302-317.

Sellers, R. M., \& Shelton, J. N. (2003). The Role of Racial Identity in Perceived Racial Discrimination. Journal of Personality and Social Psychology, 84(5), 1079-1092.

Serpe, R., \& Stryker, S. (1987). The Construction of Self and Reconstruction of Social Relationships. In E. J. Lawler \& B. Markovsky (Eds.), Advances in Group Processes (4th ed., pp. 41-66). Greenwich, CT: JAI Press. 
Sharpe, T. T., Harrison, K. M., \& Dean, H. D. (2010). Summary of CDC Consultation to address social determinants of health for prevention of disparities in HIV/AIDS, viral hepatitis, sexually transmitted diseases, and tuberculosis. Public Health Reports, 125(Suppl 4), 11-15.

Sherbourne, C. D., \& Stewart, A. L. (1991). THE MOS Social Support Survey. Social Science and Medicine, 32(6), 705-714.

Shrier, L. A., Harris, S. K., Sternberg, M., \& Beardsless, W. R. (2001). Association of Depression, Self-Esteem, and Substance Use with Sexual Risk Among Adolescents. Preventive Medicine, 33(3), 179-189.

Simoni, J. M., Sehgal, S., \& Walters, K. L. (2004). Triangle of Risk: Urban American Indian Women's Sexual Trauma , Injection Drug Use , and HIV Sexual Risk Behaviors, 8(1).

Simoni, J. M., Walters, K. L., Balsam, K. F., \& Meyers, S. B. (2006). Victimization, substance use, and HIV risk behaviors among gay/bisexual/two-spirit and heterosexual American Indian Men in New York City. American Journal of Public Health, 96(12), 2240-5. doi:10.2105/AJPH.2004.054056

Smith, L. T. (1999). Decolonizing Methodologies: Research and Indigenous Peoples. New York, NY: Zed Books.

Smith, L. T. (2005). On Tricky Ground: Researching the Native in the Age of Uncertainty. In N. K. Denzin \& Y. S. Lincoln (Eds.), The SAGE Handbook of Qualitative Research (3rd ed., pp. 152-171). Walnut Creek, CA: SAGE.

Smith, L. T. (2010). Indigenous Knowledges. In International Network of Indigenous Health Knowledge and Development: Knowing Our Roots: Indigenous Medicines, Health Knowledges, and Best Practices. Port Madison Indian Reservation, Suquamish Nation.

Stall, R. D., Paul, J. P., Greenwood, G., Pollack, L. M., Bein, E., \& Crosby, G. M. (2001). Alcohol Use, Drug Use, and Alcohol-Related Problems Among Men Who Have Sex With Men: The Urban Men's Health Study. Addiction, 96, 1589-1601.

Stall, R., Mills, T. C., Williamson, J., Hart, T., Greenwood, G., Paul, J., ... Catania, J. a. (2003). Association of co-occurring psychosocial health problems and increased vulnerability to HIV/AIDS among urban men who have sex with men. American Journal of Public Health, 93(6), 939-42. Retrieved from http://www.pubmedcentral.nih.gov/articlerender.fcgi?artid=1447874\&tool=p mcentrez\&rendertype=abstract 
Stokes, J. P., \& Peterson, J. L. (1998). Homophobia, Self-Esteem, and Risk for HIV among African American Men who have sex with Men. AIDS Education and Prevention, 10(3), 278-292. Retrieved from http://psycnet.apa.org/psycinfo/1998-10002-006

Strathdee, S. A., Hogg, R. S., Martindale, S. L., Cornelisses, P. G. A., Craib, K. J. P., Montaner, J. S. G., ... Schechter, M. T. (1998). Determinants of Sexual Risk-Takin Among Young HIV-Negative Gay and Bisexual Men. Journal of Acquired Immune Deficiency Syndromes and Human Retrovirology, 19, 61-66.

Stryker, S. (1987). Identity Theory: Developments and Extensions. In T. Honess \& K. Yardley (Eds.), Self and Identity: Psychosocial Perspectives (pp. 89-194). London: Wiley.

Suarez, T., \& Miller, J. (2001). Negotiating Risks In Context: A Perspective on Unprotected Anal Intercourse And Barebacking Among Men Who Have Sex WIth Men - Where Do We Go From Here? Archives of Sexual Behavior, 30, 287300 .

Sue, D. W., Capodilupo, C. M., Torino, G. C., Bucceri, J. M., Holder, A. M. B., Nadal, K. L., \& Esquilin, M. (2007). Racial microaggressions in everyday life: implications for clinical practice. The American Psychologist, 62(4), 271-86. doi:10.1037/0003066X.62.4.271

Sullivan, P., Chu, S., Fleming, P., \& Ward, J. (1997). Changes in AIDS incidence for men who have sex with men. United States 1990-1995. AIDS, 11, 1641-1646.

Sullivan, P. S., Drake, A. J., \& Sanchez, T. H. (2006). Prevalence Of Treatment Optimism-Related Risk Beahvior And Associated Factors Among Men Who Have Sex With Men in 11 States. AIDS and Behavior, 11, 123-129.

Sullivan, P. S., Salazar, L., Buchbinder, S., \& Sanchez, T. H. (2009). Estimating The Proportion Of HIV Transmissions From Main Sex Partners Among Men Who Have Sex With Men In Five US Cities. AIDS, 23(9), 1153-1162.

Szymanski, D. M. (2009). Examining potential moderators of the link between heterosexist events and gay and bisexual men's psychological distress. Journal of Counseling Psychology, 56(1), 142-151. doi:10.1037/0022-0167.56.1.142

Tafoya, T. (1996). Native Two-Spirit People. In R. P. Cabaj \& T. S. Stein (Eds.), Textbook of Homosexuality and Mental Health (pp. 603-617). Washington, D.C.: American Pssychiatric Press. 
Tebaldi, E., \& Elmslie, B. (2006). Sexual Orientation and the Labour Supply. Applied Economics, 38, 549-562.

Teunis, N. (2007). Sexual objectification and the construction of whiteness in the gay male community. Culture, Health \& Sexuality, 9(3), 263-75. doi:10.1080/13691050601035597

Thurman, P. J., Vernon, I. S., \& Plested, B. (2007). Advancing HIV/AIDS prevention among American Indians through capacity building and the community readiness model. Journal of Public Health Management and Practice : JPHMP, Suppl, S49-54. Retrieved from http://www.ncbi.nlm.nih.gov/pubmed/17159467

Tinker, G. E. (1993). Missionary Conquest: The gospel and Native American cultural genocide. Minneapolis, MN: Fortress Press.

Turner, R. J., \& Lloyd, D. A. (1999). The stress process and the social distribution of depression. Journal of Health and Social Behavior, 40, 374-404.

Van De Ven, P., Kippax, S., Crawford, J. M., Rawstone, P., Prestage, G., \& Grulich, A. E. (2002). In A Minority Of Gay Men, Sexual Risk Practice Indicates Strategic Positioning For Preceived Risk Reduction Rather Than Unbridled Sex. AIDS Care, 14, 471-480.

Van Kesteren, N. M., Hospers, H. J., \& Kok, G. (2006). Sexual Risk Behavior Among HIV-Positive Men Who Have Sex With Men: A Literature Review. Patient Education and Counseling, 65, 5-20.

Vanable, P. A., Ostrow, D., McKiman, D. J., Taywaditep, K. J., \& Hope, B. A. (2000). Impact Of Combination Therapies On HIV Risk Perceptions And Sexual Risk Among HIV-Postive And HIV-Negative Gay and Bisexual Men. Health Psychology, 19, 134-145.

Varghese, B., Maher, J. E., Peterman, T. A., Branson, B. M., \& Steketee, R. W. (2002). Reducing The Risk Of Sexual HIV Transmission: Quatifying The Per-Act Risk For HIV On The Basis Of Choice Of Partner, Sex Act, and Condom Use. Sexually Transmitted Diseases, 29, 38-43.

Vernon, I. S. (2007). American Indian women, HIV/AIDS, and health disparity. Substance Use \& Misuse, 42(4), 741-52. doi:10.1080/10826080701202569 
Vittinghoff, E., Douglas, J., Judson, F., McKirnan, D., MacQueen, K., \& Buchbinder, S. (1999). Per-Contact Risk of Human Immunodeficiency Virus Transmission Between Male Sexual Partners. American Journal of Epidemiology, 150, 306311.

Vu, L., Choi, K.-H., \& Do, T. (2011). Correlates of sexual, ethnic, and dual identity: a study of young Asian and Pacific Islander men who have sex with men. AIDS Education and Prevention : Official Publication of the International Society for AIDS Education, 23(5), 423-36. doi:10.1521/aeap.2011.23.5.423

Walters, K. L. (1995). Urban AMerican Indian Identity and Psychological Wellness [Dissertation]. University of California, Los Angeles.

Walters, K. L. (1997). Urban Lesbian and Gay American Indian Identity : Implications for Mental Health Service Delivery. Journal of Gay \& Lesbian Social Services, 6(2), 43-65.

Walters, K. L. (1999). Urban American Indian Identity Attitudes and Acculturation Styles. Journal of Human Behavior in the Social Environment, 2(1/2), 163-178.

Walters, K. L., Evans-campbell, T., Simoni, J. M., Ronquillo, T., \& Bhuyan, R. (2006). "My Spirit in My Heart": Identity Experiences and Challenges Among American Indian Two-Spirit Women. Journal of Lesbian Studies, 10(1-2), 125-149. doi:10.1300/J155v10n01

Walters, K. L., Horwath, P. F., \& Simoni, J. M. (2001). Sexual Orientation Bias Experiences and Service Needs of Gay, Lesbian, Bisexual, Transgendered, and Two-Spirited American Indians. Journal of Gay \& Lesbian Social Services, 13(12), 133-149. doi:10.1300/J041v13n01_10

Walters, K. L., Simoni, J. M., \& Evans-Campbell, T. (2002). Substance Use Among American Indians and Alaska Natives: Incorporating Culture in an "Indigenist" Stress-Coping Paradigm. Public Health Reports, 117(1), S104-S117.

Walters, K. L., Simoni, J. M., \& Harris, C. (2000). Patterns and predictors of HIV risk among urban American Indians. American Indian and Alaska Native Mental Health Research (Online), 9(2), 1-21. Retrieved from http://www.ncbi.nlm.nih.gov/pubmed/11279555

Weber, L. (2010). Understanding race, class, gender, and sexuality: A conceptual framework (2nd ed.). New York, NY: Oxford University Press. 
Weinhardt, L. S. (2005). HIV Diagnosis and Risk Behavior. In S. C. Kalichman (Ed.), Positive Prevention: Reducing HIV Transmission Among People Living With HIV/AIDS (pp. 29-63). New York, NY: Kluwer Academic / Plenum Publishers.

Wiechelt, S. A., Gryczynski, J., \& Johnson, J. L. (2009). Designing HIV Prevention Interventions for Urban American Indians: Evolution of the Don't Forget Us Program. Health \& Social Work, 34(4), 301-305. Retrieved from http://hsw.oxfordjournals.org/content/34/4/301.short

Williams, D. R. (1997). Race and Health : Basic Questions, Emerging Directions. Annals of Epidemiology, 7(5), 322-333.

Williams, D. R., \& Collins, C. (1995). US Socioeconomic and Racial Differences in Health: Patterns and Explanations. Annual Review of Sociology, 21, 349-386.

Williams, D. R., Neighbors, H. W., \& Jackson, J. S. (2003). Racial/ethnic discrimination and health: findings from community studies. American Journal of Public Health, 93(2), 200-208. Retrieved from

http://www.pubmedcentral.nih.gov/articlerender.fcgi?artid=2518588\&tool=p mcentrez\&rendertype=abstract

Williams, D. R., Yu, Y., Jackson, J. S., \& Anderson, N. B. (1997). Racial Differences in Physical and Mental Health: Socio-economic Status, Stress and Discrimination. Journal of Health Psychology, 2(3), 335-51. doi:10.1177/135910539700200305

Wilson, K., \& Rosenberg, M. W. (2002). Exploring the determinants of health for First Nations peoples in Canada: Can existing frameworks acommodate traditional Activities? Social Science and Medicine, 55, 2017-2031.

Wilson, P. A, Díaz, R. M., Yoshikawa, H., \& Shrout, P. E. (2009). Drug use, interpersonal attraction, and communication: situational factors as predictors of episodes of unprotected anal intercourse among Latino gay men. AIDS and Behavior, 13(4), 691-9. doi:10.1007/s10461-008-9479-9

Wilson, P. A., \& Yoshikawa, H. (2004). Experiences of and Responses to Social Discrimination among Asian and Pacific Islander Gay Men: Their Relationship to HIV Risk. AIDS Education and Prevention, 16(1), 68-83.

Wilton, L., Herbst, J. H., Painter, T. M., Alvarez, M. E., Johnson, W. D., Carey, J. W., ... Lucas, B. (2009). Efficacy of an HIV/ STI prevention intervention for black men who have sex with men: findings from the Many Men, Many Voices (3MV) project. AIDS and Behavior, 13(3), 532-544. 
Wolitski, R. J. (2005). The Emergence Of Barebacking Among Gay Men In The United States: A Public Health Perspective. Journal of Gay and Lesbian Psychology, 9, 13-38.

Wolitski, R. J., Bailey, C. J., O'Leary, A., Gomez, C. A., \& Parsons, J. T. (2003). SelfPerceived Responsibility of HIV-Seropositive Men Who Have Sex With Men for Preventing HIV Transmission. AIDS and Behavior, 7, 363-372.

Wolitski, R. J., Flores, S. A., O'Leary, A., Bimbi, D. S., \& Gomez, C. A. (2007). Beliefs About Personal and Partner Responsibility among HIV-Seropositive Men Who Have Sex With Men: Measurement and Association with Transmission Risk Behavior. AIDS and Behavior, 11, 676-686.

Wolitski, R. J., Valdiserri, R. O., \& Stall, R. (2008). Health Disparities Affecting Gay and Bisexual Men in the United States: An Introduction. In R. J. Wolitski, R. O. Valdiserri, \& R. Stall (Eds.), Unequal Opportunity: Health Disparities Affecting Gay and Bisexual Men in the United States (1st ed., pp. 3-32). New York, NY: Oxford University Press.

Yellow Horse Brave Heart, M. (2003). The Historical Trauma Response among Natives and Its Relationship with Substance Abuse: A Lakota Illustration. Journal of Psychoactive Drugs, 35(1), 7-13.

Yen, I., \& Ragland, D. (1999). Racial Discrimination and Alcohol-Related Behavior in Urban Transit Operators - Findings from the San Francisco Muni Health and Safety Study. Public Health Reports, 114, 448-458. Retrieved from http://www.ncbi.nlm.nih.gov/pmc/articles/PMC1308517/

Yoshikawa, H., Wilson, P. A., \& Chae, D. H. (2002). Demographic, Psychosocial, and Neighborhood Predictors of HIV Risk and Testing Among Asian / Pacific Islander Men Who Have Sex With Men in the US. In XIV International AIDS Conference. Barcelona.

Yoshikawa, H., Wilson, P. A.-D., Chae, D. H., \& Cheng, J.-F. (2004). Do Family and Friendship Networks Protect Against the Influence of Discrimination on Mental Health and HIV Risk Among Asian and Pacific Islander Gay Men? AIDS Education and ..., 16(1), 84-100. Retrieved from http://guilfordjournals.com/doi/abs/10.1521/aeap.16.1.84.27719

Zablotska, I. B., Crawford, J., Imrie, J., Prestage, G., Jin, F., Grulich, A., \& Kippax, S. (2009). Increases In Unprotected Anal Intercourse With Serodiscordant Causal Sex Partners Among HIV-Negative Gay Men In Sydney. AIDS and Behavior, 13, 638-644. 
Zierler, S., \& Krieger, N. (1998). HIV infection in women: Social inequalities as determinants of risk. Critical Public Health, 8(1), 13-32. Retrieved from http://www.tandfonline.com/doi/abs/10.1080/09581599808409209 


\section{APPENDIX A: Microaggression Scales}

\begin{tabular}{|c|c|}
\hline $\begin{array}{l}\text { Racial } \\
\text { Microaggressi } \\
\text { on } \\
\text { Scale } \\
33 / 33\end{array}$ & $\begin{array}{l}\text { We are interested in your experiences with discrimination and common } \\
\text { hassles. } \\
\text { Please indicate which number best describes how much that experience or } \\
\text { situation distressed or bothered you EVER IN YOUR LIFE. }\end{array}$ \\
\hline \multirow{18}{*}{$\begin{array}{l}\text { 0. Not at all } \\
\text { 1. A little bit } \\
\text { 2. Moderately } \\
\text { 3. Quite a bit } \\
\text { 4. Extremely }\end{array}$} & $\begin{array}{l}\text { IN YOUR LIFETIME, how much were you distressed or bothered by unfair } \\
\text { treatment by your bosses or supervisors because you are Native? }\end{array}$ \\
\hline & $\begin{array}{l}\text { IN YOUR LIFETIME, how much were you distressed or bothered by unfair } \\
\text { treatment from people in helping or social service jobs, such as a therapist or } \\
\text { social worker? }\end{array}$ \\
\hline & $\begin{array}{l}\text { IN YOUR LIFETIME, how much were you distressed or bothered by unfair } \\
\text { treatment by institutions, such as schools, police, social services, or } \\
\text { immigration because you are Native? }\end{array}$ \\
\hline & $\begin{array}{l}\text { IN YOUR LIFETIME, how much were you distressed or bothered by wanting to } \\
\text { verbally respond to someone for being anti-Indian, but didn't? }\end{array}$ \\
\hline & $\begin{array}{l}\text { IN YOUR LIFETIME, how much were you distressed or bothered by being } \\
\text { accused of not doing your share of the work because you are Native? }\end{array}$ \\
\hline & $\begin{array}{l}\text { IN YOUR LIFETIME, how much were you distressed or bothered by anti-Indian } \\
\text { statements made to you? }\end{array}$ \\
\hline & $\begin{array}{l}\text { IN YOUR LIFETIME, how much were you distressed or bothered by having to } \\
\text { take drastic steps such as quitting your job or moving away to deal with some } \\
\text { racist thing that was done to you? }\end{array}$ \\
\hline & $\begin{array}{l}\text { IN YOUR LIFETIME, how much were you distressed or bothered by being } \\
\text { called a racist name like Chief, Wahoo, Squaw, or Pocohantas? }\end{array}$ \\
\hline & $\begin{array}{l}\text { IN YOUR LIFETIME, how much were you distressed or bothered by getting } \\
\text { into an argument with non-Natives about something they said that was racist } \\
\text { towards Native Americans? }\end{array}$ \\
\hline & $\begin{array}{l}\text { IN YOUR LIFETIME, how much were you distressed or bothered by being } \\
\text { made fun of or picked-on because you are Native? }\end{array}$ \\
\hline & $\begin{array}{l}\text { IN YOUR LIFETIME, how much were you distressed or bothered by being } \\
\text { asked if you are a "real Indian" by a non-Native person? }\end{array}$ \\
\hline & $\begin{array}{l}\text { IN YOUR LIFETIME, how much were you distressed or bothered by being } \\
\text { asked to prove your Indianness or authenticity by a non-Native person? }\end{array}$ \\
\hline & $\begin{array}{l}\text { IN YOUR LIFETIME, how much were you distressed or bothered by being } \\
\text { asked by a stranger if he or she could touch you because you are Native? }\end{array}$ \\
\hline & $\begin{array}{l}\text { IN YOUR LIFETIME, how much were you distressed or bothered by being } \\
\text { asked by a non-Native stranger if you could perform a ceremony or contact a } \\
\text { medicine person for him or her? }\end{array}$ \\
\hline & $\begin{array}{l}\text { IN YOUR LIFETIME, how much were you distressed or bothered by feeling } \\
\text { "invisible" to non-Natives? }\end{array}$ \\
\hline & $\begin{array}{l}\text { IN YOUR LIFETIME, how much were you distressed or bothered by teaching } \\
\text { "Indian 101" to non-Natives to make your point or be heard? }\end{array}$ \\
\hline & $\begin{array}{l}\text { IN YOUR LIFETIME, how much were you distressed or bothered by being } \\
\text { asked to change your Native appearance or apparel by your employer or } \\
\text { agency, for example, being asked to cut your hair? }\end{array}$ \\
\hline & $\begin{array}{l}\text { IN YOUR LIFETIME, how much were you distressed or bothered by hearing } \\
\text { from non-Natives how surprisingly articulate, well read, or good your } \\
\text { language skills are? }\end{array}$ \\
\hline
\end{tabular}




\begin{tabular}{|c|c|}
\hline & $\begin{array}{l}\text { IN YOUR LIFETIME, how much were you distressed or bothered by Non- } \\
\text { Natives stating to you that you "don't look or act Indian"? }\end{array}$ \\
\hline & $\begin{array}{l}\text { IN YOUR LIFETIME, how much were you distressed or bothered by hearing } \\
\text { discussion by instructors or other persons in authority about Indians as if they } \\
\text { no longer exist. }\end{array}$ \\
\hline & $\begin{array}{l}\text { IN YOUR LIFETIME, how much were you distressed or bothered by feeling } \\
\text { stereotyped or boxed-in to a certain way of being "Native" by non-Native } \\
\text { persons? }\end{array}$ \\
\hline & $\begin{array}{l}\text { IN YOUR LIFETIME, how much were you distressed or bothered by being hit, } \\
\text { kicked, or physically attacked because you are Native? }\end{array}$ \\
\hline & $\begin{array}{l}\text { IN YOUR LIFETIME, how much were you distressed or bothered by being } \\
\text { trailed or followed in a store because you are Native? }\end{array}$ \\
\hline & $\begin{array}{l}\text { IN YOUR LIFETIME, how much were you distressed or bothered by being told } \\
\text { to "lighten up" or "get a sense of humor" about Indian mascots or logos } \\
\text { (example, Cleveland Indians or Tomahawk Chop)? }\end{array}$ \\
\hline & $\begin{array}{l}\text { IN YOUR LIFETIME, how much were you distressed or bothered by being } \\
\text { mistaken by non-Natives as a racial group other than Native? }\end{array}$ \\
\hline & $\begin{array}{l}\text { IN YOUR LIFETIME, how much were you distressed or bothered by hearing } \\
\text { racist statements such as "Indian giver" among others? }\end{array}$ \\
\hline & $\begin{array}{l}\text { IN YOUR LIFETIME, how much were you distressed or bothered by having } \\
\text { non-Native strangers speak a foreign language to you such as Spanish or } \\
\text { Chinese? }\end{array}$ \\
\hline & $\begin{array}{l}\text { IN YOUR LIFETIME, how much were you distressed or bothered by being told } \\
\text { that Indians are conquered and should stop trying to live in the past? }\end{array}$ \\
\hline & $\begin{array}{l}\text { IN YOUR LIFETIME, how much were you distressed or bothered by being told } \\
\text { by a non-Native person that he or she was an Indian in a past life or that their } \\
\text { grandmother was a Cherokee princess? }\end{array}$ \\
\hline & $\begin{array}{l}\text { IN YOUR LIFETIME, how much were you distressed or bothered by being told } \\
\text { by non-Natives how they wished they were Indian too? }\end{array}$ \\
\hline & $\begin{array}{l}\text { IN YOUR LIFETIME, how much were you distressed or bothered by being told } \\
\text { by non-Natives that they felt a spiritual connection to Indian people? }\end{array}$ \\
\hline & $\begin{array}{l}\text { IN YOUR LIFETIME, how much were you distressed or bothered by being told } \\
\text { you are "paranoid" by non-Natives? }\end{array}$ \\
\hline & $\begin{array}{l}\text { IN YOUR LIFETIME, how much were you distressed or bothered by being told } \\
\text { by non-Natives how "lucky" you are to be Indian? }\end{array}$ \\
\hline $\begin{array}{l}\text { Heterosexist } \\
\text { Microaggressi }\end{array}$ & $\begin{array}{l}\text { For the next set of questions, please indicate how much were you distressed } \\
\text { or bothered by the following experiences EVER in your life. }\end{array}$ \\
\hline on Scale & IN YOUR LIFETIME, how much were you distressed or bothered by .... \\
\hline $32 / 32$ & encountering white people who identify as two-spirit? \\
\hline 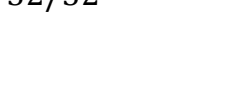 & $\begin{array}{l}\text { hearing people use tribal two-spirit terms (e.g., winkte, nadle) in a pejorative } \\
\text { way? }\end{array}$ \\
\hline 0. Not at all & non-Native LGBT people assuming that you are spiritually gifted? \\
\hline 1. A little bit & non-Native people assuming that you can speak for all two-spirit people? \\
\hline $\begin{array}{l}\text { 2. Moderately } \\
\text { 3. Quite a bit }\end{array}$ & $\begin{array}{l}\text { non-Native people stating that they feel a "spiritual connection" to two-spirit } \\
\text { people? }\end{array}$ \\
\hline 4. Very much so & non-Native people appropriating two-spirit names? \\
\hline & $\begin{array}{l}\text { being excluded from LGBT groups, events, or programs because you are two- } \\
\text { spirit? }\end{array}$ \\
\hline & your tribe or Native community denying your existence? \\
\hline & being told that being LGBT is a "White thing"? \\
\hline & d to leave ceremonies because you are two-spirit? \\
\hline
\end{tabular}




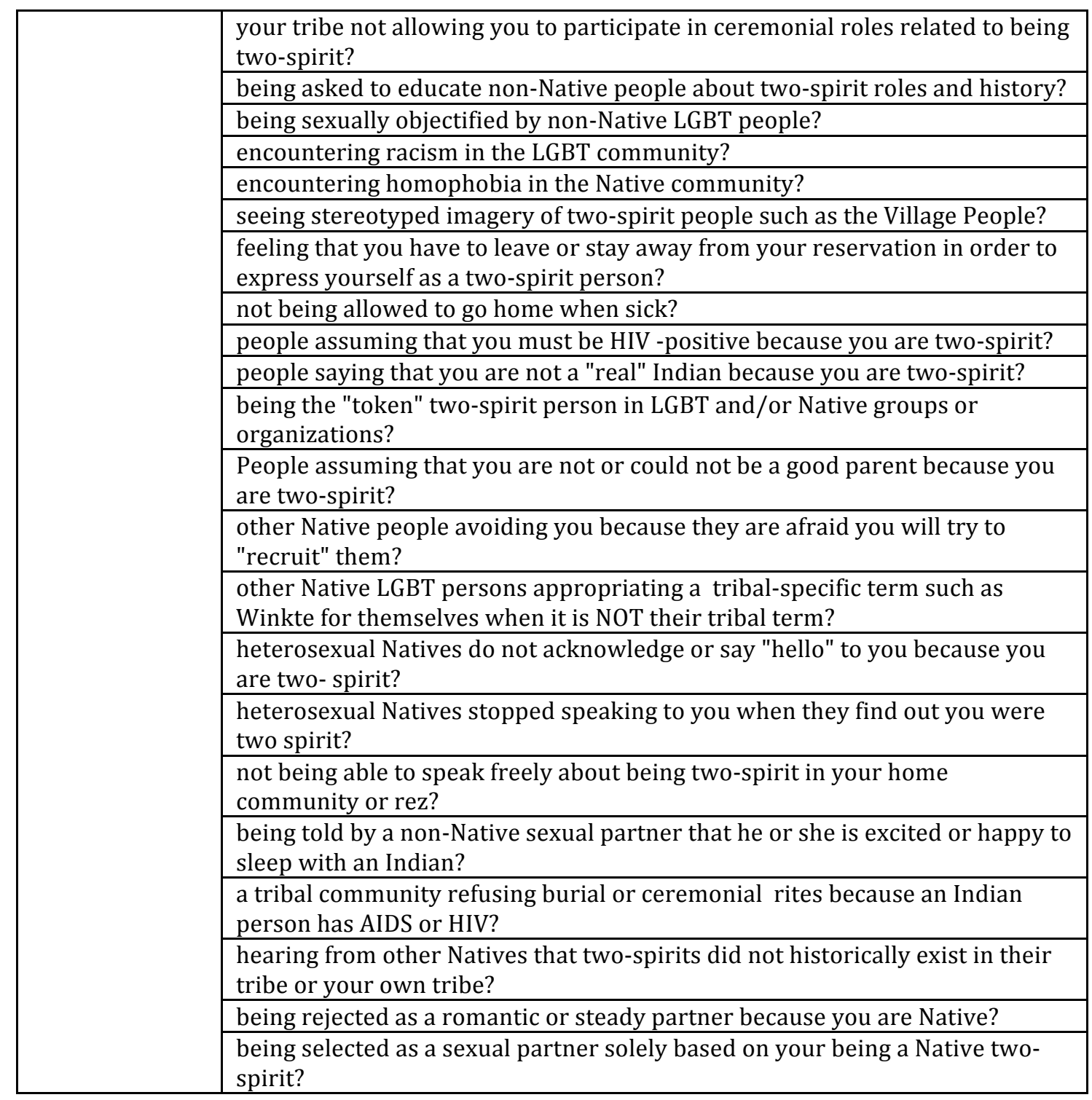




\section{APPENDIX B: Center for Epidemiologic Studies - Short Depression Scale}

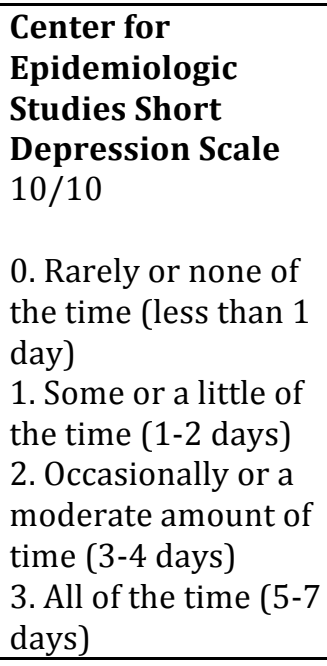

The next set of questions ask about ways you might have felt or behaved in the past week. As you go through the questions, try and remember that they are only asking about THE PAST WEEK.

During the past week...

I was bothered by things that usually don't bother me.

I had trouble keeping my mind on what I was doing.

I felt depressed.

I felt that everything I did was an effort.

I felt hopeful about the future.

I felt fearful.

My sleep was restless.

I was happy.

I felt lonely.

I could not "get going". 


\section{APPENDIX C: Perceived Availability of Social Support Scale}

\begin{tabular}{|c|c|c|}
\hline \multirow{2}{*}{$\begin{array}{l}\text { Perceive } \\
\text { d } \\
\text { availabil } \\
\text { ity of } \\
\text { social } \\
\text { support }\end{array}$} & $\begin{array}{l}\text { About how many close friends and close relatives do you } \\
\text { have (people you feel at ease with and can talk to about } \\
\text { what is on your mind)? }\end{array}$ & (number: 0-99) \\
\hline & $\begin{array}{l}\text { People sometimes look to others for companionship, } \\
\text { assistance, or other types of support. How often is someone } \\
\text { available to provide the following types of support to you if } \\
\text { you need it? }\end{array}$ & \multirow{21}{*}{$\begin{array}{l}\text { 0. None of the time } \\
\text { 1. A little of the time } \\
\text { 2. Some of the time } \\
\text { 3. Most of the time } \\
\text { 4. All of the time }\end{array}$} \\
\hline \multirow{20}{*}{$\begin{array}{l}\text { 20-item } \\
\text { medical } \\
\text { Outcome } \\
\text { s Study - } \\
\text { Social } \\
\text { Support } \\
\text { (MOS-SS) } \\
\text { Sherburn } \\
\text { e and } \\
\text { Stewart } \\
1991\end{array}$} & This type of support is available if you need it ... & \\
\hline & $\begin{array}{l}\text { Someone you can count on to listen to you when you need } \\
\text { to talk }\end{array}$ & \\
\hline & Someone to give you good advice about a crisis & \\
\hline & Someone to give you a ride to the doctor if you needed it & \\
\hline & Someone who shows you love and affection & \\
\hline & Someone to have a good time with & \\
\hline & $\begin{array}{l}\text { Someone to give you information to help you understand a } \\
\text { situation }\end{array}$ & \\
\hline & $\begin{array}{l}\text { Someone to confide in or talk to about yourself or your } \\
\text { problems }\end{array}$ & \\
\hline & Someone who hugs you & \\
\hline & Someone to get together with for relaxation & \\
\hline & Someone whose advice you really want & \\
\hline & $\begin{array}{l}\text { Someone to do things with to help you get your mind off } \\
\text { things }\end{array}$ & \\
\hline & Someone to help you if you were confined to bed & \\
\hline & Someone to help with daily chores if you were sick & \\
\hline & Someone to share your most private worries and fears with & \\
\hline & $\begin{array}{l}\text { Someone to turn to for suggestions about how to deal with } \\
\text { a personal problem }\end{array}$ & \\
\hline & Someone to do something enjoyable with & \\
\hline & Someone who understands your problems & \\
\hline & Someone to love and make you feel wanted & \\
\hline & $\begin{array}{l}\text { Someone to prepare your meals if you were unable to do it } \\
\text { yourself }\end{array}$ & \\
\hline
\end{tabular}




\section{APPENDIX D: LGBT and Native Identity Scales}

\begin{tabular}{|c|c|}
\hline \multirow{4}{*}{$\begin{array}{l}\text { LGBT Identity } \\
\text { and } \\
\text { Acceptance } \\
31 / 31\end{array}$} & $\begin{array}{l}\text { For each of the following statements, choose the response that best indicates } \\
\text { your experience as LGBTT-S. }\end{array}$ \\
\hline & I prefer to keep my same-sex romantic relationships rather private \\
\hline & $\begin{array}{l}\text { I will never be able to accept my sexual orientation until all of the people in my } \\
\text { life have accepted me }\end{array}$ \\
\hline & I would rather be straight if I could \\
\hline \multirow{28}{*}{$\begin{array}{l}\text { 1. Strongly } \\
\text { disagree } \\
\text { 2. Moderately } \\
\text { disagree } \\
\text { 3. Somewhat } \\
\text { disagree } \\
\text { 4. Neither } \\
\text { agree nor } \\
\text { disagree } \\
\text { 5. Somewhat } \\
\text { agree } \\
\text { 6. Moderately } \\
\text { agree } \\
\text { 7. Strongly } \\
\text { agree }\end{array}$} & Coming out to my friends and family has been a very lengthy process \\
\hline & I'm not totally sure what my sexual orientation is \\
\hline & $\begin{array}{l}\text { I keep careful control over who knows about my same-sex romantic } \\
\text { relationships }\end{array}$ \\
\hline & I often wonder whether others judge me for being LGBT-TS \\
\hline & I am glad to be a LGBTT-S person \\
\hline & I look down on heterosexuals \\
\hline & I keep changing my mind about my sexual orientation \\
\hline & My private sexual behavior is nobody's business \\
\hline & $\begin{array}{l}\text { I can't feel comfortable knowing that other Native people judge me negatively for } \\
\text { being LGBTT-S }\end{array}$ \\
\hline & $\begin{array}{l}\text { I can't feel comfortable knowing that non-Native people judge me negatively for } \\
\text { being LGBTT-S }\end{array}$ \\
\hline & Homosexual lifestyles are not as fulfilling as heterosexual lifestyles \\
\hline & Admitting to myself that I'm a LGBTT-S person has been a very painful process \\
\hline & If you are not careful about whom you come out to, you can get very hurt \\
\hline & Being a LGBTT-S person makes me feel insecure around straight people \\
\hline & I'm proud to be part of the two-spirit community \\
\hline & I'm proud to be part of the LGBT community \\
\hline & Developing as a LGBTT-S person has been a fairly natural process for me \\
\hline & I can't decide whether I am bisexual or (lesbian/gay) \\
\hline & I think very carefully before coming out to someone \\
\hline & $\begin{array}{l}\text { I think a lot about how my sexual orientation affects the way other Native people } \\
\text { see me }\end{array}$ \\
\hline & $\begin{array}{l}\text { I think a lot about how my sexual orientation affects the way non-Native people } \\
\text { see me }\end{array}$ \\
\hline & Admitting to myself that I'm a LGBTT-S person has been a very slow process \\
\hline & $\begin{array}{l}\text { Straight people have boring lives compared with lesbian, gay, and bisexual } \\
\text { people }\end{array}$ \\
\hline & My sexual orientation is a very personal and private matter \\
\hline & I wish I were heterosexual \\
\hline & I get very confused when I try to figure out my sexual orientation \\
\hline & I have felt comfortable with my sexual identity just about from the start \\
\hline & I have a hard time reconciling being LGBTT-S with being Native \\
\hline \multirow{7}{*}{$\begin{array}{l}\text { Self } \\
\text { Actualization } \\
\text { Subscale - } \\
\text { Urban } \\
\text { American } \\
\text { Indian } \\
\text { Identity } \\
\text { Attitudes }\end{array}$} & $\begin{array}{l}\text { Please indicate how much you disagree or agree with the following statements } \\
\text { about your social and cultural attitudes. }\end{array}$ \\
\hline & I feel good about my Indian identity. \\
\hline & Indian culture has many strengths. \\
\hline & I feel a spiritual connection to Indian land. \\
\hline & I feel uneasy about my Indian identity. \\
\hline & I feel at peace with my Indian identity. \\
\hline & I honor my ancestors in my relationships with other Indians. \\
\hline
\end{tabular}




\begin{tabular}{|l|l|}
\hline (UAIIS) & I get a long better with White people than with Indians \\
\cline { 2 - 2 } & I am not sure what being Indian means to me. \\
\cline { 2 - 2 } & Traditional Indian ways are not for me. \\
\cline { 2 - 2 } & Usually, I try to pass as non-Indian when I'm around non-Indians. \\
\cline { 2 - 2 } 1. Strongly & I prefer hanging out in the White community rather than the Indian community. \\
\cline { 2 - 2 } disagree & I have many strengths because I am Indian. \\
\cline { 2 - 2 } $\begin{array}{l}\text { 2. Somewhat } \\
\text { disagree }\end{array}$ & I often think I would rather be a White person. \\
\cline { 2 - 2 } $\begin{array}{l}\text { 3. Somewhat } \\
\text { agree }\end{array}$ & I want to learn more my about my cultural heritage \\
\cline { 2 - 2 } 4. Strongly & Most of the time I am uncomfortable around Indians \\
\cline { 2 - 2 } agree & Indians have many strengths \\
\cline { 2 - 2 } & I am proud to be Indian \\
\hline
\end{tabular}




\section{APPENDIX E: LGBT and Native Community Participation Measures}

\begin{tabular}{|c|c|c|}
\hline \multirow{9}{*}{$\begin{array}{l}\text { Ceremonial } \\
\text { and Cultural } \\
\text { Activity } \\
\text { Participation }\end{array}$} & & \\
\hline & $\begin{array}{l}\text { How often do you participate in traditional } \\
\text { ceremonies (not including prayer or meditation)? }\end{array}$ & \multirow{8}{*}{$\begin{array}{l}\text { 1. Once a day or more } \\
\text { 2. 2-6 times a week } \\
\text { 3. Once a week } \\
\text { 4. 1-3 times a month } \\
\text { 5. Less than } 12 \text { times a year } \\
\text { 6. Never }\end{array}$} \\
\hline & $\begin{array}{l}\text { How often do you use ceremonial tobacco for } \\
\text { prayer? }\end{array}$ & \\
\hline & $\begin{array}{l}\text { How often do you use sage, sweetgrass, cedar, } \\
\text { corn pollen, or any other sacred plants/medicines } \\
\text { for purification or cleansing, or prayer or } \\
\text { protection? }\end{array}$ & \\
\hline & $\begin{array}{l}\text { How often do you use a spirit broom for cleansing } \\
\text { and/or prayer? }\end{array}$ & \\
\hline & $\begin{array}{l}\text { How often do you smoke a pipe or roll and smoke } \\
\text { tobacco for prayer? }\end{array}$ & \\
\hline & $\begin{array}{l}\text { How often do you do Indian singing and/or } \\
\text { drumming? }\end{array}$ & \\
\hline & $\begin{array}{l}\text { How often do you use traditional treatments, } \\
\text { remedies, and teas (such as sweetgrass, cedar, } \\
\text { black drink, "Indian tea," or herbs such as bear } \\
\text { root or black root, among others)? }\end{array}$ & \\
\hline & $\begin{array}{l}\text { How often do you participate in Long House or } \\
\text { Big House ceremonies? }\end{array}$ & \\
\hline \multirow{4}{*}{$\begin{array}{l}\text { Connection } \\
\text { to and } \\
\text { Participation } \\
\text { in LGBT } \\
\text { Community }\end{array}$} & & \\
\hline & $\begin{array}{l}\text { How often do you go to LGBT bars, clubs, or } \\
\text { parties that are not specifically for two-spirits? }\end{array}$ & \multirow{3}{*}{$\begin{array}{l}\text { 1.At least once a day } \\
\text { 2. 2-6 times a week } \\
\text { 3. Once a week } \\
\text { 4. 1-3 times a month } \\
\text { 5. Several times a year } \\
\text { 6. Once a year or less } \\
\text { 7. Never }\end{array}$} \\
\hline & $\begin{array}{l}\text { How often do you attend LGBT events (support } \\
\text { groups, meetings, political rallies, parades, etc.) } \\
\text { that are not specifically for two-spirits }\end{array}$ & \\
\hline & $\begin{array}{l}\text { How often do you participate in or visit non- } \\
\text { Native LGBT web-sites, on-line chat rooms, or e- } \\
\text { mail discussion groups? }\end{array}$ & \\
\hline
\end{tabular}

